\title{
Scalar products of Bethe vectors in the 8-vertex model
}

\section{N. Slavnov, A. Zabrodin and A. Zotov}

Steklov Mathematical Institute of Russian Academy of Sciences, Gubkina str. 8, Moscow, 119991, Russia

E-mail: nslavnov@mi-ras.ru, zabrodin@itep.ru, zotov@mi-ras.ru

ABSTRACT: We obtain a determinant representation of normalized scalar products of onshell and off-shell Bethe vectors in the inhomogeneous 8-vertex model. We consider the case of rational anisotropy parameter and use the generalized algebraic Bethe ansatz approach. Our method is to obtain a system of linear equations for the scalar products, prove its solvability and solve it in terms of determinants of explicitly known matrices.

KeYwords: Bethe Ansatz, Lattice Integrable Models

ARXIV EPRINT: 2005.11224 


\section{Contents}

1 Introduction $\quad 1$

2 Inhomogeneous 8-vertex model 4

2.1 The $R$-matrix 4

2.2 Intertwining vectors 8

$\begin{array}{lll}2.3 & \text { Vacuum vectors } & 10\end{array}$

3 The generalized algebraic Bethe ansatz $\quad 12$

$\begin{array}{ll}3.1 \text { The permutation relations } & 13\end{array}$

$\begin{array}{lll}3.2 & \text { Right eigenvectors } & 15\end{array}$

$\begin{array}{lll}3.3 & \text { Left eigenvectors } & 18\end{array}$

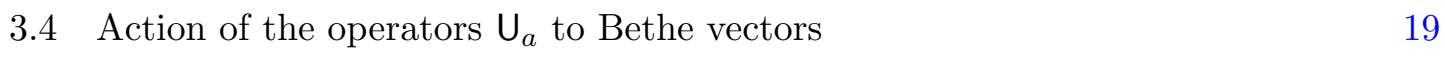

$\begin{array}{ll}3.5 & \text { The case of rational } \eta \\ 3.6 & 20\end{array}$

$\begin{array}{ll}3.6 & \text { Dependence of the eigenvectors on } s, t\end{array}$

4 The $Q$-operator and the sum rule $\quad 22$

4.1 Construction of the $Q$-operator 22

4.2 The sum rule 24

5 Scalar products of Bethe vectors $\quad \mathbf{2 5}$

5.1 The notation 25

5.2 A system of linear equations for scalar products 26

$\begin{array}{lll}5.3 & \text { Transformation of the system and solvability } & 27\end{array}$

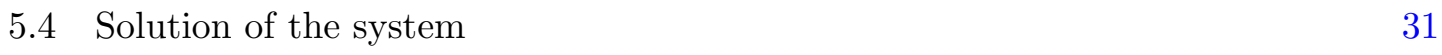

5.5 Trying to fix the ambiguity 32

$\begin{array}{lll}5.6 & \text { The result for scalar products } & 35\end{array}$

$\begin{array}{lll}5.7 & \text { The selection rule } & 35\end{array}$

5.8 Orthogonality and norm of on-shell Bethe vectors 36

$\begin{array}{lll}5.9 & \text { Normalized scalar products } & 38\end{array}$

6 Concluding remarks $\quad 39$

$\begin{array}{ll}\text { A Null-vector } & 40\end{array}$

$\begin{array}{ll}\text { B } \text { The case } N=2 & 41\end{array}$

C Free fermions $\quad 45$

$\begin{array}{ll}\text { D List of notations } & 48\end{array}$ 


\section{Introduction}

The study of low-dimensional strongly correlated systems is of great importance and interest. Among the numerous physical low-dimensional models, a special role is played by the 8 -vertex model [1-4], which is closely related or in some sense equivalent to the completely anisotropic $X Y Z$ Heisenberg magnet [5]. This model is completely integrable, because the corresponding matrix of Boltzmann weights (the $R$-matrix) satisfies the Yang-Baxter equation, and transfer matrices commute for any values of the arguments. However, unlike the 6-vertex model, in the 8-vertex model, the total flow through the vertex, generally speaking, is not conserved. In the language of the $X Y Z$ chain, this leads to the fact that the third component of the total spin is no longer an integral of motion. All this implies significant difficulties in the study of this model.

The spectral problem for the 8-vertex model was solved by $\mathrm{R}$. Baxter via the $Q$ operator method [6-10], which subsequently found wide applications. Further research on the 8-vertex model took place in several directions. Besides studying the properties of the $T-Q$ equation and the solutions of Bethe equations [11-20], it is also worth mentioning the study of the underlying quantum algebras [21-25]. A number of works were devoted to the study of correlation functions of the 8-vertex model [26-31]. However, there are still many open questions in this direction.

In 1979, the Quantum Inverse Scattering Method (QISM) [32, 33] was applied to the $X Y Z$ Heisenberg chain in paper [34]. This required the development of some generalization of the algebraic Bethe ansatz, since this method in its original formulation is not applicable to the $X Y Z$ chain. The generalized algebraic Bethe ansatz allows us to obtain Bethe equations that determine the spectrum of the Hamiltonian, as well as construct the eigenvectors of the transfer matrix. The question arises of the applicability of this method to the calculation of form factors and correlation functions. This way looks very attractive, since the application of the standard algebraic Bethe ansatz to this problem has been developed quite well to date. Let us briefly recall the main features of this approach.

The calculation of correlation functions within the QISM consists of several stages. At the first stage, it is necessary to derive the action of local operators on physical states (on-shell Bethe vectors). This is achieved either within the framework of the composite model [35], or by explicitly solving the quantum inverse problem [36]. The latter allows us to express explicitly local spin operators via elements of the monodromy matrix. For the $X Y Z$ chain, the quantum inverse problem was solved in [37] (see also [38]).

At the next step, it is necessary to calculate the arising scalar products of Bethe vectors. In these scalar products, one of the vectors is still an eigenvector of the Hamiltonian (or equivalently, an eigenvector of the transfer matrix), while the second, generally speaking, is not (an off-shell Bethe vector). Attempts to calculate norms and the scalar products directly with the help of the algebra satisfied by elements of the quantum monodromy matrix show that this is a difficult combinatorial problem.

The history of the problem is as follows. The first result is Gaudin's hypothesis which goes back to $1972[39,40]$ (see also the later work [41]) about norms of eigenvectors of the Hamiltonian of the Bose-gas with point-like interaction. This hypothesis was proved 
in 1982 by Korepin [42] in the framework of the quantum inverse scattering method for a sufficiently wide class of models. It states that the squared norm of eigenvectors of the Hamiltonian is given by determinant of an $m \times m$ matrix ( $m$ is the number of excitations) whose explicit form is restored from the form of Bethe equations. In 1989, for models with the 6-vertex $R$-matrix, a compact determinant formula was proved [43] for scalar products of two Bethe vectors one of which is on-shell and another one is an arbitrary off-shell Bethe vector. The original method to obtain this result was a complicated combinatorial analysis of the structure of scalar products and application of recurrence relations for them. In 1998, Kitanine, Maillet and Terras [36] obtained this result by a different method and showed that the matrix elements of the matrix participating in the determinant representation of scalar products are expressed through derivatives of eigenvalues of the transfer matrix. Later similar results were obtained for models with the 6 -vertex $R$-matrix with non-periodic boundary conditions [44-47]. In paper [48], a determinant representation was obtained for scalar products in the elliptic solid-on-solid (SOS) model, which is closely related to the 8-vertex model.

Recently, a new method was proposed in [49], which avoids all combinatorial difficulties and allows one to reduce the calculation of scalar products of on-shell and off-shell Bethe vectors in models with the 6 -vertex $R$-matrix to solving a system of linear equations. This method explains why the scalar products of on-shell and off-shell Bethe vectors have determinant representations.

The existence of determinant representations for scalar products allows us to directly proceed to the calculation of correlation functions. Here we should mention the method based on the use of auxiliary quantum operators (dual fields) [50-52], as well as the method based on the representation for correlation functions in the form of multiple integrals [5357]. However, for today, the most powerful and relatively simple approach is the method of form factor expansion. In particular, a significant successes have been achieved along this path in the study of the correlation functions of the $X X Z$ chain and the model of onedimensional bosons (the Lieb-Liniger model). Both analytical $[58,59]$ and numerical [60-63] results were obtained.

The lack of compact determinant representations for scalar products in the 8-vertex model (or the $X Y Z$ chain) is a serious obstacle for the study of the correlation functions within the framework of the QISM. At the same time, the method of [49] relies solely on the formula for the action of the transfer matrix on the Bethe vectors. For the $X Y Z$ chain, this formula was obtained in pioneer work [34]. Therefore, this approach is carried over without significant changes to the case of models with the 8-vertex $R$-matrix.

In this paper, we use the approach suggested in [49]. Namely, we obtain a system of linear equations whose solutions are the scalar products of the on-shell and off-shell Bethe vectors of the inhomogeneous 8-vertex model. We find these solutions in terms of determinants (minors of the matrix of the linear system). At this stage, we basically follow the strategy of [49]. A significant difference appears at the final stage. A feature of this method is that the above system of linear equations is homogeneous. Therefore, its solutions contain an ambiguity which must be fixed. In models with the 6 -vertex (trigonometric or rational) $R$-matrix, this is achieved by considering a special particular 
case, in which the scalar product is reduced to the partition function of the 6-vertex model with the domain wall boundary conditions which has a determinant representation [42, 64]. An analogue of this result in the case of the 8-vertex model is not known (see, however, papers [65, 66], where the representation of the partition function of the elliptic SOS model with the domain wall boundary conditions as a finite linear combination of determinants was obtained). Therefore, the study of this particular case for the models with the 8-vertex $R$-matrix does not lead to the desired results. Instead, we fix the freedom in the resulting solution using the quasiperiodic transformation properties of Bethe vectors under sifts of the variables by periods. Despite of this method allows one to fix the ambiguity only partially, the residual arbitrariness disappears in the normalized expressions.

Let us present here the main result. We consider the inhomogeneous 8-vertex model or the $X Y Z$ spin- $\frac{1}{2}$ chain on an even number of sites $N=2 n$ with a rational anisotropy parameter $\eta=2 P / Q$. Let $\left\langle\Psi_{\nu}\left(\left\{v_{i}\right\}\right)\right|$ be the (dual) eigenvector of the transfer matrix $\mathrm{T}(u)$ with the eigenvalue $T_{\nu}\left(u ;\left\{v_{i}\right\}\right)$ explicitly given by (3.23) below (here $\nu=0,1, \ldots, Q-1$, and the parameters $v_{1}, \ldots, v_{n}$ satisfy the Bethe equations) and let $\left|\Psi_{\mu}\left(\left\{u_{i}\right\}\right)\right\rangle$ be an off-shell Bethe vector with arbitrary parameters $u_{1}, \ldots, u_{n}$. Let also define

$$
V=\sum_{i=1}^{n} v_{i}, \quad U=\sum_{i=1}^{n} u_{i},
$$

and $r=V-U$. Our main result is the following determinant formula for the scalar product for the on-shell and off-shell Bethe vectors:

$$
\begin{aligned}
\left\langle\Psi_{\nu}\left(\left\{v_{i}\right\}\right) \mid \Psi_{\mu}\left(\left\{u_{i}\right\}\right)\right\rangle= & \phi_{1}^{(\nu \mu)}(r) \phi_{2}^{(\nu)}\left(\left\{v_{i}\right\}\right) \\
& \times \frac{\prod_{a, b=1}^{n} \theta_{1}\left(u_{a}-v_{b} \mid \tau\right)}{\prod_{p<q} \theta_{1}\left(u_{p}-u_{q} \mid \tau\right) \theta_{1}\left(v_{q}-v_{p} \mid \tau\right)} \operatorname{det}_{1 \leq j, k \leq n} T_{j k}^{(\nu \mu)}(r),
\end{aligned}
$$

where the matrix $T_{j k}^{(\nu \mu)}(r)$ is given by

$$
T_{j k}^{(\nu \mu)}(r)=\frac{\theta_{1}^{\prime}(0 \mid \tau) \theta_{1}\left(u_{k}-v_{j}+r \mid \tau\right)}{\theta_{1}\left(u_{k}-v_{j} \mid \tau\right) \theta_{1}(r \mid \tau)}\left(T_{\nu}\left(u_{k} ; v_{1}, \ldots, v_{n}\right)-T_{\mu}\left(u_{k}, v_{1}, \ldots, v_{j}-r, \ldots, v_{n}\right)\right) .
$$

In (1.1), (1.2), $\theta_{1}(z \mid \tau)$ is the odd Jacobi theta function with modular parameter $\tau \in \mathbb{C}$ with $\operatorname{Im} \tau>0$. The function $\phi_{1}^{(\nu \mu)}(r)$ in (1.1) is known explicitly (see (5.49), (5.50) below). The function $\phi_{2}^{(\nu)}\left(\left\{v_{i}\right\}\right)$ can not be fixed by our method. However, it does not enter the expression for specially normalized scalar products

$$
\frac{\left\langle\Psi_{\nu}\left(\left\{v_{i}\right\}\right) \mid \Psi_{\mu}\left(\left\{u_{i}\right\}\right)\right\rangle}{\left\langle\Psi_{\nu}\left(\left\{v_{i}\right\}\right) \mid \Psi_{\nu}\left(\left\{v_{i}\right\}\right)\right\rangle} .
$$

We argue that only such expressions are essential for finding correlation functions.

The paper is organized as follows. In section 2 we introduce the main objects of the 8-vertex model and $X Y Z$ spin chain: the $R$-matrix (in the elliptic parametrization), the $L$-operator, the quantum monodromy matrix and the transfer matrix. After that we study how the $R$-matrix acts on the tensor products of some specially parameterized vectors in 
$\mathbb{C}^{2}$ and introduce vacuum vectors for gauge-transformed $L$-operators. section 3 explains how the generalized algebraic Bethe ansatz works for the construction of eigenvectors of the transfer matrix. First, the commutation relations for the elements of the gauge-transformed quantum monodromy matrix are derived and then their algebra is used to construct right and left (dual) eigenvectors, basically in the same way as in [34]. In section 4 we recall the alternative method of diagonalization of the transfer matrix based on the $Q$-operator and derive the sum rule for Bethe roots for the inhomogeneous model. This section is included for completeness and is not directly related to what follows. The main content of the paper is contained in section 5 , where we obtain a homogeneous system of linear equations for the scalar products of Bethe vectors, prove its solvability and solve it in terms of determinants. We also prove that our result implies orthogonality of on-shell Bethe vectors.

There are also three appendices and a list of notations. In appendix A we show that our result implies that some special Bethe vectors are in fact null-vectors. Appendix B is devoted to a detailed account of the simplest case $N=2$. In appendix $\mathrm{C}$ we show that in the case $\eta=\frac{1}{2}$ (the case of free fermions) even more explicit results can be obtained.

\section{Inhomogeneous 8-vertex model}

Since the $X Y Z$ spin- $\frac{1}{2}$ chain and the 8-vertex model are equivalent, below we mostly talk about the latter for definiteness. We stress that we consider an inhomogeneous model only for reasons of generality. All formulas below allow a smooth homogeneous limit.

\section{$2.1 \quad$ The $R$-matrix}

The matrix of Boltzmann weights of the 8 -vertex model (the $R$-matrix) has a natural elliptic parametrization [4]. In order to write it explicitly, we use the Jacobi theta functions

$$
\begin{aligned}
& \theta_{1}(u \mid \tau)=-i \sum_{k \in \mathbb{Z}}(-1)^{k} q^{\left(k+\frac{1}{2}\right)^{2}} e^{\pi i(2 k+1) u} \\
& \theta_{2}(u \mid \tau)=\sum_{k \in \mathbb{Z}} q^{\left(k+\frac{1}{2}\right)^{2}} e^{\pi i(2 k+1) u} \\
& \theta_{3}(u \mid \tau)=\sum_{k \in \mathbb{Z}} q^{k^{2}} e^{2 \pi i k u} \\
& \theta_{4}(u \mid \tau)=\sum_{k \in \mathbb{Z}}(-1)^{k} q^{k^{2}} e^{2 \pi i k u}
\end{aligned}
$$

where $\tau \in \mathbb{C}, \operatorname{Im} \tau>0$, and $q=e^{\pi i \tau}$. Let us mention the infinite product representation

$$
\theta_{1}(u \mid \tau)=2 q^{\frac{1}{4}} \sin \pi u \prod_{n \geq 1}\left(1-q^{2 n}\right)\left(1-q^{2 n} e^{2 \pi i u}\right)\left(1-q^{2 n} e^{-2 \pi i u}\right) .
$$

Similar infinite product representations exist also for the other theta-functions which are connected with $\theta_{1}(u \mid \tau)$ by the formulas

$$
\begin{array}{ll}
\theta_{2}(u \mid \tau)=\theta_{1}\left(u+\frac{1}{2} \mid \tau\right), & \theta_{3}(u \mid \tau)=q^{\frac{1}{4}} e^{\pi i u} \theta_{1}\left(u+\frac{\tau+1}{2} \mid \tau\right) \\
\theta_{4}(u \mid \tau)=-i q^{\frac{1}{4}} e^{\pi i u} \theta_{1}\left(u+\frac{\tau}{2} \mid \tau\right) . &
\end{array}
$$


It is seen from here that $\theta_{1}$ and $\theta_{2}$ become respectively sin and $\cos$ as $q \rightarrow 0$ while $\theta_{3}$ and $\theta_{4}$ become constants (equal to 1 ). The function $\theta_{1}$ is odd while the other three are even. The theta functions satisfy a large number of non-trivial identities which are used below without comments. Most of these identities with proofs can be found in [67].

Let

$$
\sigma_{0}=\left(\begin{array}{ll}
1 & 0 \\
0 & 1
\end{array}\right), \quad \sigma_{1}=\left(\begin{array}{ll}
0 & 1 \\
1 & 0
\end{array}\right), \quad \sigma_{2}=\left(\begin{array}{rr}
0 & -i \\
i & 0
\end{array}\right), \quad \sigma_{3}=\left(\begin{array}{rr}
1 & 0 \\
0 & -1
\end{array}\right)
$$

be Pauli matrices, $\sigma_{ \pm}=\frac{1}{2}\left(\sigma_{1} \pm i \sigma_{2}\right)$. The Baxter's $R$-matrix of the symmetric 8 -vertex model in the elliptic parametrization has the form

$$
\mathrm{R}(u)=\mathrm{R}(u ; \eta, \tau)=\sum_{a=0}^{3} W_{a}(u) \sigma_{a} \otimes \sigma_{a},
$$

where

$$
W_{a}(u)=W_{a}(u ; \eta, \tau)=\theta_{1}(\eta \mid \tau) \frac{\theta_{5-a}\left(u+\frac{\eta}{2} \mid \tau\right)}{2 \theta_{5-a}\left(\frac{\eta}{2} \mid \tau\right)}
$$

and the index of the theta functions is understood modulo 4 (for example, $\theta_{5}(u \mid \tau)=$ $\left.\theta_{1}(u \mid \tau)\right)$. This $R$-matrix acts in the tensor product $V_{1} \otimes V_{2}\left(V_{i} \cong \mathbb{C}^{2}\right)$ and can be also denoted as $\mathrm{R}_{12}(u)=\sum_{a=0}^{3} W_{a}(u) \sigma_{a}^{(1)} \sigma_{a}^{(2)}$. (Pictorially, the $R$-matrix is represented as the vertex + formed by intersection of two lines, the first space being associated with the horizontal line and the second one with the vertical line.) In the matrix form we have:

$$
\begin{aligned}
\mathrm{R}(u) & =\left(\begin{array}{cccc}
W_{0}(u)+W_{3}(u) & 0 & 0 & W_{1}(u)-W_{2}(u) \\
0 & W_{0}(u)-W_{3}(u) & W_{1}(u)+W_{2}(u) & 0 \\
0 & W_{1}(u)+W_{2}(u) & W_{0}(u)-W_{3}(u) & 0 \\
W_{1}(u)-W_{2}(u) & 0 & 0 & W_{0}(u)+W_{3}(u)
\end{array}\right) \\
& =\left(\begin{array}{cccc}
a^{8 \mathrm{v}}(u) & 0 & 0 & d^{8 \mathrm{v}}(u) \\
0 & b^{8 \mathrm{v}}(u) & c^{8 \mathrm{v}}(u) & 0 \\
0 & c^{8 \mathrm{v}}(u) & b^{8 \mathrm{v}}(u) & 0 \\
d^{8 \mathrm{v}}(u) & 0 & 0 & a^{8 \mathrm{v}}(u)
\end{array}\right),
\end{aligned}
$$

where

$$
\begin{aligned}
a^{8 \mathrm{v}}(u) & =\frac{2 \theta_{4}(\eta \mid 2 \tau) \theta_{1}(u+\eta \mid 2 \tau) \theta_{4}(u \mid 2 \tau)}{\theta_{2}(0 \mid \tau) \theta_{4}(0 \mid 2 \tau)}, \\
b^{8 \mathrm{v}}(u) & =\frac{2 \theta_{4}(\eta \mid 2 \tau) \theta_{4}(u+\eta \mid 2 \tau) \theta_{1}(u \mid 2 \tau)}{\theta_{2}(0 \mid \tau) \theta_{4}(0 \mid 2 \tau)}, \\
c^{8 \mathrm{v}}(u) & =\frac{2 \theta_{1}(\eta \mid 2 \tau) \theta_{4}(u+\eta \mid 2 \tau) \theta_{4}(u \mid 2 \tau)}{\theta_{2}(0 \mid \tau) \theta_{4}(0 \mid 2 \tau)}, \\
d^{8 \mathrm{v}}(u) & =\frac{2 \theta_{1}(\eta \mid 2 \tau) \theta_{1}(u+\eta \mid 2 \tau) \theta_{1}(u \mid 2 \tau)}{\theta_{2}(0 \mid \tau) \theta_{4}(0 \mid 2 \tau)} .
\end{aligned}
$$

It is easy to see that when the spectral parameter $u$ is shifted by the quasiperiods 1 and $\tau$, the $R$-matrix transforms as follows:

$$
\begin{aligned}
& \mathrm{R}_{12}(u+1)=-\sigma_{3}^{(1)} \mathrm{R}_{12}(u) \sigma_{3}^{(1)} \\
& \mathrm{R}_{12}(u+\tau)=-e^{-\pi i(2 u+\eta+\tau)} \sigma_{1}^{(1)} \mathrm{R}_{12}(u) \sigma_{1}^{(1)} .
\end{aligned}
$$


It can be shown that this $R$-matrix satisfies the Yang-Baxter equation [7]

$$
\mathrm{R}_{12}\left(u_{1}-u_{2}\right) \mathrm{R}_{13}\left(u_{1}\right) \mathrm{R}_{23}\left(u_{2}\right)=\mathrm{R}_{23}\left(u_{2}\right) \mathrm{R}_{13}\left(u_{1}\right) \mathrm{R}_{12}\left(u_{1}-u_{2}\right)
$$

and commutes with $\sigma_{a} \otimes \sigma_{a}$ :

$$
\sigma_{a} \otimes \sigma_{a} \mathrm{R}(u)=\mathrm{R}(u) \sigma_{a} \otimes \sigma_{a}, \quad a=1,2,3 .
$$

Below we also need the following properties of the $R$-matrix (2.5):

$$
\begin{aligned}
\mathrm{R}_{12}(-u ;-\eta, \tau) & =-\mathrm{R}_{12}(u ; \eta, \tau), \\
\mathrm{R}_{12}^{t_{1} t_{2}}(u) & =\mathrm{R}_{12}(u), \\
\mathrm{R}_{12}(u-\eta ; \eta, \tau) & =e^{\pi i(2 u-\eta+\tau)} \mathrm{R}_{12}^{t_{1}}(u+\tau+1 ;-\eta, \tau),
\end{aligned}
$$

where $t_{i}$ means transposition in the $i$-th space.

In the limit $\tau \rightarrow+i \infty(q \rightarrow 0)$ the elliptic $R$-matrix degenerates into the standard trigonometric $R$-matrix of the 6 -vertex model:

$$
\mathrm{R}(u) \rightarrow 2 q^{\frac{1}{4}}\left(\begin{array}{cccc}
\sin \pi(u+\eta) & 0 & 0 & 0 \\
0 & \sin \pi u & \sin \pi \eta & 0 \\
0 & \sin \pi \eta & \sin \pi u & 0 \\
0 & 0 & 0 & \sin \pi(u+\eta)
\end{array}\right)+O\left(q^{\frac{5}{4}}\right)
$$

The $R$-matrix (2.5) can be also represented in the form of the $L$-operator

$$
\mathrm{L}(u)=\left(\begin{array}{cc}
W_{0}(u) \sigma_{0}+W_{3}(u) \sigma_{3} & W_{1}(u) \sigma_{1}-i W_{2}(u) \sigma_{2} \\
W_{1}(u) \sigma_{1}+i W_{2}(u) \sigma_{2} & W_{0}(u) \sigma_{0}-W_{3}(u) \sigma_{3}
\end{array}\right)=\left(\begin{array}{c}
\mathrm{a}(u) \mathrm{b}(u) \\
\mathrm{c}(u) \mathrm{d}(u)
\end{array}\right),
$$

which is the $2 \times 2$ matrix whose matrix elements are operators in $\mathbb{C}^{2}$. Clearly, it is the same $R$-matrix (2.5) written as a block matrix. ${ }^{1}$ The Yang-Baxter equation for $\mathrm{R}$ is the $R L L=L L R$ relation for $\mathrm{L}$

$$
\mathrm{R}_{12}(u-v) \mathrm{L}_{1}(u) \mathrm{L}_{2}(v)=\mathrm{L}_{2}(v) \mathrm{L}_{1}(u) \mathrm{R}_{12}(u-v),
$$

where $\mathrm{L}_{1}(u)=\mathrm{L}(u) \otimes 1, \mathrm{~L}_{2}(v)=1 \otimes \mathrm{L}(v)$.

The quantum monodromy matrix of the inhomogeneous 8-vertex model is

$$
\mathcal{T}(u)=\mathrm{L}_{1}\left(u-\xi_{1}\right) \mathrm{L}_{2}\left(u-\xi_{2}\right) \ldots \mathrm{L}_{N}\left(u-\xi_{N}\right)=\left(\begin{array}{cc}
A(u) & B(u) \\
C(u) & D(u)
\end{array}\right),
$$

where complex numbers $\xi_{i}$ are inhomogeneity parameters. It is an operator in $\mathbb{C}^{2} \otimes \mathcal{H}$, $\mathcal{H}=\bigotimes_{i=1}^{N} V_{i}, V_{i} \cong \mathbb{C}^{2}$. Here $\mathrm{L}_{j}(u)$ is given by the formula (2.11), where the $\sigma$-matrices $\sigma_{a}^{(j)}$ act in the $j$-th copy of $\mathbb{C}^{2}$ associated with the $j$-th site of the lattice. The operators $A(u), B(u), C(u), D(u)$ act in the space $\mathcal{H}$. We consider the case of even $N=2 n$, otherwise

\footnotetext{
${ }^{1}$ Usually in the literature the $L$-operator differs from the $R$-matrix by a shift of the spectral parameter $u \rightarrow u-\eta / 2$. We do not make this shift.
} 
solvability of the model is problematic. Equations (2.7) imply the following properties of the quantum monodromy matrix:

$$
\begin{aligned}
& \mathcal{T}(u+1)=\sigma_{3} \mathcal{T}(u) \sigma_{3}=\left(\begin{array}{rr}
A(u) & -B(u) \\
-C(u) & D(u)
\end{array}\right), \\
& \mathcal{T}(u+\tau)=e^{-\pi i c(u)} \sigma_{1} \mathcal{T}(u) \sigma_{1}=e^{-\pi i c(u)}\left(\begin{array}{rr}
D(u) & C(u) \\
B(u) & A(u)
\end{array}\right),
\end{aligned}
$$

where

$$
c(u)=N(2 u+\eta+\tau)-2 \sum_{k=1}^{N} \xi_{k} .
$$

It follows from (2.12) and (2.13) that the quantum monodromy matrix satisfies the $R T T=$ $T T R$ relation

$$
\mathrm{R}_{12}(u-v) \mathcal{T}_{1}(u) \mathcal{T}_{2}(v)=\mathcal{T}_{2}(v) \mathcal{T}_{1}(u) \mathrm{R}_{12}(u-v),
$$

This relation implies that the transfer matrices

$$
\begin{aligned}
\mathrm{T}(u) & =\operatorname{tr}_{0}\left(\mathrm{R}_{01}\left(u-\xi_{1}\right) \mathrm{R}_{02}\left(u-\xi_{2}\right) \ldots \mathrm{R}_{0 N}\left(u-\xi_{N}\right)\right) \\
& =\operatorname{tr}\left(\mathrm{L}_{1}\left(u-\xi_{1}\right) \mathrm{L}_{2}\left(u-\xi_{2}\right) \ldots \mathrm{L}_{N}\left(u-\xi_{N}\right)\right)=\operatorname{tr} \mathcal{T}(u)=A(u)+D(u)
\end{aligned}
$$

commute for any values of the spectral parameter $u$. The transfer matrix is an operator in the space $\mathcal{H}$. For the solution of the model one is interested in eigenvectors and eigenvalues of the transfer matrix.

It follows from (2.9) that the transfer matrix commutes with the operators

$$
\mathrm{U}_{a}=\left(\sigma_{a}\right)^{\otimes N} .
$$

Note that the operators $\mathrm{U}_{a}$ commute with each other:

$$
\mathrm{U}_{a} \mathrm{U}_{b}=\left(\sigma_{a} \sigma_{b}\right)^{\otimes N}=(-1)^{N}\left(\sigma_{b} \sigma_{a}\right)^{\otimes N}=\mathrm{U}_{b} \mathrm{U}_{a}
$$

(because $N$ is an even number). Therefore, the problem is to find common eigenvectors of the transfer matrix and the operators $\mathrm{U}_{a}$.

The homogeneous 8-vertex model (when all $\xi_{i}$ are equal to 0 ) is closely related to the $X Y Z$ spin- $\frac{1}{2}$ chain. The connection goes as follows: the Hamiltonian $H^{\mathrm{XYZ}}$ of the $X Y Z$ spin chain is contained in the commuting family of operators $\mathrm{T}(u)$ in the following way:

$$
\left.\partial_{u} \log \mathrm{T}(u)\right|_{u=0}=\frac{\theta_{1}^{\prime}(0 \mid \tau)}{2 \theta_{1}(\eta \mid \tau)} H^{\mathrm{XYZ}}+J_{0} N \mathbf{1},
$$

where $J_{0}=\frac{1}{2} \theta_{1}^{\prime}(\eta \mid \tau) / \theta_{1}(\eta \mid \tau)$, and $\mathbf{1}$ is the identity operator. The Hamiltonian of the $X Y Z$ chain is given by

$$
H^{\mathrm{XYZ}}=\sum_{j=1}^{N}\left(J_{1} \sigma_{1}^{(j)} \sigma_{1}^{(j+1)}+J_{2} \sigma_{2}^{(j)} \sigma_{2}^{(j+1)}+J_{3} \sigma_{3}^{(j)} \sigma_{3}^{(j+1)}\right)
$$


with the constants

$$
J_{1}=\frac{\theta_{4}(\eta \mid \tau)}{\theta_{4}(0 \mid \tau)}, \quad J_{2}=\frac{\theta_{3}(\eta \mid \tau)}{\theta_{3}(0 \mid \tau)}, \quad J_{3}=\frac{\theta_{2}(\eta \mid \tau)}{\theta_{2}(0 \mid \tau)} .
$$

The transfer matrix of the homogeneous 8-vertex model is a generating function for conserved quantities of the $X Y Z$ spin- $\frac{1}{2}$ chain.

\section{$2.2 \quad$ Intertwining vectors}

The $L$-operator of the 8 -vertex model does not have a vacuum vector, i.e. a vector annihilated by the operator $\mathrm{c}(u)$, because the matrix $\mathrm{c}(u)$ is non-degenerate for almost all $u$. This fact makes it impossible to apply directly the algebraic Bethe ansatz method used for the solution of the 6 -vertex model. Instead, one can apply the so-called generalized algebraic Bethe ansatz [34]. The key ingredient of the generalized algebraic Bethe ansatz for the 8 -vertex model is the rule of the action of the $R$-matrix (2.5) to some special vectors.

Let us introduce a family of vectors

$$
|\phi(s)\rangle=\left(\begin{array}{c}
\theta_{1}(s \mid 2 \tau) \\
\theta_{4}(s \mid 2 \tau)
\end{array}\right),
$$

where $s$ is a complex parameter. They are called intertwining vectors. The covector orthogonal to $|\phi(s)\rangle$ is

$$
\left\langle\phi^{\perp}(s)\right|=\left(-\theta_{4}(s \mid 2 \tau), \theta_{1}(s \mid 2 \tau)\right)=i e^{-\pi i\left(s+\frac{\tau}{2}\right)}\langle\phi(s+\tau+1)|
$$

and the scalar product $\left\langle\phi^{\perp}(t) \mid \phi(s)\right\rangle$ is given by

$$
\begin{aligned}
\left\langle\phi^{\perp}(t) \mid \phi(s)\right\rangle & =\theta_{1}\left(\frac{1}{2}(t-s) \mid \tau\right) \theta_{2}\left(\frac{1}{2}(t+s) \mid \tau\right) \\
& =2 \frac{\theta_{1}\left(\frac{1}{2}(t-s) \mid 2 \tau\right) \theta_{4}\left(\frac{1}{2}(t-s) \mid 2 \tau\right) \theta_{2}\left(\frac{1}{2}(t+s) \mid 2 \tau\right) \theta_{3}\left(\frac{1}{2}(t+s) \mid 2 \tau\right)}{\theta_{2}(0 \mid 2 \tau) \theta_{3}(0 \mid 2 \tau)} .
\end{aligned}
$$

Using the identities for the theta functions, one can prove the following important identity for the intertwining vectors:

$$
\mathrm{R}(u)|\phi(s+\eta)\rangle \otimes|\phi(s-u)\rangle=\theta_{1}(u+\eta \mid \tau)|\phi(s)\rangle \otimes|\phi(s-u+\eta)\rangle
$$

or, indicating explicitly the spaces where the vectors live:

$$
\mathrm{R}_{12}(u)|\phi(s+\eta)\rangle_{1}|\phi(s-u)\rangle_{2}=\theta_{1}(u+\eta \mid \tau)|\phi(s)\rangle_{1}|\phi(s-u+\eta)\rangle_{2} .
$$

Note that when one acts by the $R$-matrix to the tensor product of two vectors, one in general obtains a linear combination of pure tensor products. The situation when one gets just one tensor product term as in (2.23) is exceptional. This property was called by Baxter "passing of a pair of vectors through the vertex" [4] and it played a very important role in his solution of the 8-vertex model. The vectors that satisfy this property are parameterized 
by points of an elliptic curve which is uniformized by the parameter $s$. This is the origin of the parameter $s$ in (2.23).

Let us give some other useful versions of identity (2.24). Changing $u \rightarrow-u, \eta \rightarrow-\eta$ in (2.24) and using (2.10), we arrive at

$$
\mathrm{R}_{12}(u)|\phi(s-\eta)\rangle_{1}|\phi(s+u)\rangle_{2}=\theta_{1}(u+\eta \mid \tau)|\phi(s)\rangle_{1}|\phi(s+u-\eta)\rangle_{2} .
$$

Shifting $s \rightarrow s+\tau+1$ and transposing in the both spaces, we also get the transposed version of equation (2.24):

$$
\left\langle\phi ^ { \perp } ( s + \eta ) | _ { 1 } \left\langle\left.\phi^{\perp}(s-u)\right|_{2} \mathrm{R}_{12}(u)=\theta_{1}(u+\eta \mid \tau)\left\langle\phi ^ { \perp } ( s ) | _ { 1 } \left\langle\left.\phi^{\perp}(s-u+\eta)\right|_{2} .\right.\right.\right.\right.
$$

Shifting $u \rightarrow u-\xi$ and then $s \rightarrow s+u$ in (2.24) and taking the scalar product of both sides with the covector $\left\langle\phi^{\perp}(s+u)\right|$, we get:

$$
\left\langle\left.\phi^{\perp}(s+u)\right|_{1} \mathrm{R}_{12}(u-\xi) \mid \phi(s+u+\eta)\right\rangle_{1}|\phi(s+\xi)\rangle_{2}=0,
$$

where $\xi$ is an additional arbitrary parameter. Here the operator $\left\langle\phi^{\perp}(s+u)\left|{ }_{1} \mathrm{R}_{12}(u-\xi)\right| \phi(s+\right.$ $u+\eta)\rangle_{1}$ acts in the vertical space (the space number 2). Taking the scalar product of (2.24) with the covector $\left\langle\phi^{\perp}(t)\right|$, we obtain

$$
\left\langle\left.\phi^{\perp}(t)\right|_{1} \mathrm{R}_{12}(u) \mid \phi(s+\eta)\right\rangle_{1}|\phi(s-u)\rangle_{2}=\theta_{1}(u+\eta \mid \tau)\left\langle\phi^{\perp}(t) \mid \phi(s)\right\rangle|\phi(s-u+\eta)\rangle_{2}
$$

or, what is the same but with an additional parameter $\xi$ introduced by the shift $u \rightarrow u-\xi$,

$$
\frac{\left\langle\left.\phi^{\perp}(t-u)\right|_{1} \mathrm{R}_{12}(u-\xi) \mid \phi(s+u+\eta)\right\rangle_{1}}{\left\langle\phi^{\perp}(t-u) \mid \phi(s+u)\right\rangle}|\phi(s+\xi)\rangle_{2}=\theta_{1}(u-\xi+\eta \mid \tau) \mid \phi(s+\xi+\eta\rangle_{2} .
$$

Shifting the arguments in (2.24) and changing $\eta \rightarrow-\eta$, using the property (2.10) and transposing in the first space, we obtain the following important corollary:

$$
\left\langle\left.\phi^{\perp}(s)\right|_{1} \mathrm{R}_{12}(u) \mid \phi(s-u)\right\rangle_{2}=\theta_{1}(u \mid \tau)\left\langle\left.\phi^{\perp}(s+\eta)\right|_{1} \mid \phi(s-u-\eta)\right\rangle_{2}
$$

or, what is the same but with an additional parameter $\xi$,

$$
\left\langle\left.\phi^{\perp}(s+u)\right|_{1} \mathrm{R}_{12}(u-\xi) \mid \phi(s+\xi)\right\rangle_{2}=\theta_{1}(u-\xi \mid \tau)\left\langle\left.\phi^{\perp}(s+u+\eta)\right|_{1} \mid \phi(s+\xi-\eta)\right\rangle_{2} .
$$

We stress that $\left\langle\left.\ldots\right|_{1} \mid \ldots\right\rangle_{2}$ here is not a scalar product but the tensor product of the vector and covector (which live in difference spaces). Taking the scalar product with the vector $|\phi(t-u+\eta)\rangle_{1}$ in the first space, we can write this identity in the following form:

$$
\frac{\left\langle\left.\phi^{\perp}(s+u)\right|_{1} \mathrm{R}_{12}(u-\xi) \mid \phi(t-u+\eta)\right\rangle_{1}}{\left\langle\phi^{\perp}(s+u+\eta) \mid \phi(t-u+\eta)\right\rangle}|\phi(s+\xi)\rangle_{2}=\theta_{1}(u-\xi \mid \tau)|\phi(s+\xi-\eta)\rangle_{2} .
$$

Let us now give a more general identity for the intertwining vectors which can be proved basically in the same way as $(2.24)$ :

$$
\begin{aligned}
\mathrm{R}_{12}(u)|\phi(s+\eta)\rangle_{1}|\phi(t-u)\rangle_{2}= & \frac{\theta_{1}(\eta \mid \tau) \theta_{2}\left(\frac{1}{2}(s+t)-u \mid \tau\right)}{\theta_{2}\left(\frac{1}{2}(s+t) \mid \tau\right)}|\phi(t)\rangle_{1}|\phi(s+u+\eta)\rangle_{2} \\
& +\frac{\theta_{1}(u \mid \tau) \theta_{2}\left(\frac{1}{2}(s+t)+\eta \mid \tau\right)}{\theta_{2}\left(\frac{1}{2}(s+t) \mid \tau\right)}|\phi(s)\rangle_{1}|\phi(t-u-\eta)\rangle_{2} .
\end{aligned}
$$


It provides a rule of how the $R$-matrix acts on the tensor products of two arbitrary vectors. At $t=s$ (2.32) coincides with (2.24) (this can be seen after using an identity for the theta functions). Substituting $u \rightarrow-u, \eta \rightarrow-\eta$, we also obtain:

$$
\begin{aligned}
\mathrm{R}_{12}(u)|\phi(s-\eta)\rangle_{1}|\phi(t+u)\rangle_{2}= & \frac{\theta_{1}(\eta \mid \tau) \theta_{2}\left(\frac{1}{2}(s+t)+u \mid \tau\right)}{\theta_{2}\left(\frac{1}{2}(s+t) \mid \tau\right)}|\phi(t)\rangle_{1}|\phi(s-u-\eta)\rangle_{2} \\
& +\frac{\theta_{1}(u \mid \tau) \theta_{2}\left(\frac{1}{2}(s+t)-\eta \mid \tau\right)}{\theta_{2}\left(\frac{1}{2}(s+t) \mid \tau\right)}|\phi(s)\rangle_{1}|\phi(t+u+\eta)\rangle_{2} .
\end{aligned}
$$

Equations (2.24), (2.25), (2.32), (2.33) can be unified in the "intertwining relation" between the $R$-matrix and the collection of Boltzmann weights of a IRF-type model. Introduce the vectors

$$
\begin{aligned}
& \left|\phi_{k}^{k+1}(u)\right\rangle=\left|\phi\left(s-u+k \eta+\frac{\eta}{2}\right)\right\rangle, \\
& \left|\phi_{k+1}^{k}(u)\right\rangle=\left|\phi\left(s+u+k \eta+\frac{\eta}{2}\right)\right\rangle,
\end{aligned}
$$

then the above mentioned equations can be compactly written as

$$
\mathrm{R}_{12}(u-v)\left|\phi_{k}^{k^{\prime}}(u)\right\rangle_{1}\left|\phi_{k^{\prime}}^{k^{\prime \prime}}(v)\right\rangle_{2}=\sum_{l}\left|\phi_{l}^{k^{\prime \prime}}(u)\right\rangle_{1}\left|\phi_{k}^{l}(v)\right\rangle_{2} W\left[\begin{array}{cc}
k & k^{\prime} \\
l & k^{\prime \prime}
\end{array}\right](u-v),
$$

where $W\left[\begin{array}{ll}k & k^{\prime} \\ l & k^{\prime \prime}\end{array}\right](u)=0$ unless $\left|k-k^{\prime}\right|=\left|k^{\prime}-k^{\prime \prime}\right|=\left|l-k^{\prime \prime}\right|=|l-k|=1$. The non-zero weights are:

$$
\begin{aligned}
& W\left[\begin{array}{cc}
k & k \pm 1 \\
k \pm 1 & k \pm 2
\end{array}\right](u)=\theta_{1}(u+\eta \mid \tau) \\
& W\left[\begin{array}{cc}
k & k \pm 1 \\
k \pm 1 & k
\end{array}\right](u)=\frac{\theta_{1}(\eta \mid \tau) \theta_{2}(s+k \eta \mp u \mid \tau)}{\theta_{2}(s+k \eta \mid \tau)} \\
& W\left[\begin{array}{cc}
k & k \pm 1 \\
k \mp 1 & k
\end{array}\right](u)=\frac{\theta_{1}(u \mid \tau) \theta_{2}(s+(k \pm 1) \eta \mid \tau)}{\theta_{2}(s+k \eta \mid \tau)} .
\end{aligned}
$$

A similar intertwining relation obtained from (2.35) by transposition in both spaces holds for the corresponding covectors.

\subsection{Vacuum vectors}

Let us consider the gauge transformation of the $L$-operator

$$
\mathrm{L}_{k}^{\prime}\left(u, \xi_{k}\right)=M_{k+l-1}^{-1}(u) \mathrm{L}_{k}\left(u-\xi_{k}\right) M_{k+l}(u)=\left(\begin{array}{cc}
\mathrm{a}_{k}^{\prime}(u) & \mathrm{b}_{k}^{\prime}(u) \\
\mathrm{c}_{k}^{\prime}(u) & \mathrm{d}_{k}^{\prime}(u)
\end{array}\right),
$$

where $l \in \mathbb{Z}$ is an integer parameter. The matrix $M_{k}(u)$ is given by

$$
M_{k}(u)=\left(\begin{array}{cc}
\theta_{1}\left(s_{k}+u \mid 2 \tau\right) & \gamma_{k} \theta_{1}\left(t_{k}-u \mid 2 \tau\right) \\
\theta_{4}\left(s_{k}+u \mid 2 \tau\right) & \gamma_{k} \theta_{4}\left(t_{k}-u \mid 2 \tau\right)
\end{array}\right),
$$


where $s_{k}=s+k \eta, t_{k}=t+k \eta, s, t \in \mathbb{C}$ are arbitrary parameters and

$$
\gamma_{k}=\frac{1}{\theta_{2}\left(\tau_{k} \mid 2 \tau\right) \theta_{3}\left(\tau_{k} \mid 2 \tau\right)}, \quad \tau_{k}=\frac{1}{2}\left(s_{k}+t_{k}\right) .
$$

Note that the columns of this matrix are the intertwining vectors. The inverse matrix is

$$
M_{k}^{-1}(u)=\frac{1}{\operatorname{det} M_{k}(u)}\left(\begin{array}{rr}
\gamma_{k} \theta_{4}\left(t_{k}-u \mid 2 \tau\right) & -\gamma_{k} \theta_{1}\left(t_{k}-u \mid 2 \tau\right) \\
-\theta_{4}\left(s_{k}+u \mid 2 \tau\right) & \theta_{1}\left(s_{k}+u \mid 2 \tau\right)
\end{array}\right) \text {, }
$$

where

$$
\begin{aligned}
\operatorname{det} M_{k}(u) & =-\gamma_{k}\left\langle\phi^{\perp}\left(t_{k}-u\right) \mid \phi\left(s_{k}+u\right)\right\rangle \\
& =\gamma_{k} \theta_{1}\left(\frac{1}{2}(s-t)+u \mid \tau\right) \theta_{2}\left(\tau_{k} \mid \tau\right) \\
& =2 \frac{\theta_{1}\left(\frac{1}{2}(s-t)+u \mid 2 \tau\right) \theta_{4}\left(\frac{1}{2}(s-t)+u \mid 2 \tau\right)}{\theta_{2}(0 \mid 2 \tau) \theta_{3}(0 \mid 2 \tau)} \equiv \mu(u) .
\end{aligned}
$$

Note that $\operatorname{det} M_{k}(u)=\mu(u)$ does not depend on $k$.

The gauge-transformed $L$-operator (2.37) has a local $u$-independent vacuum vector

$$
\left|\omega_{k}^{l}\right\rangle=\left(\begin{array}{c}
\theta_{1}\left(s_{k+l-1}+\xi_{k} \mid 2 \tau\right) \\
\theta_{4}\left(s_{k+l-1}+\xi_{k} \mid 2 \tau\right)
\end{array}\right)=\left|\phi\left(s_{k+l-1}+\xi_{k}\right)\right\rangle_{k} \in V_{k}
$$

which is annihilated by the left lower element $c_{k}^{\prime}(u)$ :

$$
\mathrm{c}_{k}^{\prime}(u)\left|\omega_{k}^{l}\right\rangle=0
$$

(recall that $\mathrm{c}_{k}^{\prime}(u)$ depends also on $s$ and $l$ ). This directly follows from equation (2.27) (one should put $s=s_{k+l-1}$ in the latter). In their turn, equations (2.28) and (2.31) (where one should put $\left.s=s_{k+l-1}, t=t_{k+l-1}\right)$ tell us how the operators $\mathrm{a}_{k}^{\prime}(u), \mathrm{d}_{k}^{\prime}(u)$ act to the vacuum vector:

$$
\begin{aligned}
\mathrm{a}_{k}^{\prime}(u)\left|\omega_{k}^{l}\right\rangle & =\theta_{1}\left(u-\xi_{k}+\eta \mid \tau\right)\left|\omega_{k}^{l+1}\right\rangle, \\
\mathrm{d}_{k}^{\prime}(u)\left|\omega_{k}^{l}\right\rangle & =\theta_{1}\left(u-\xi_{k} \mid \tau\right)\left|\omega_{k}^{l-1}\right\rangle .
\end{aligned}
$$

Unlike the situation in the 6-vertex model, the vacuum vector is not an eigenvector for these operators but transforms in a simple way.

The gauge-transformed quantum monodromy matrix is

$$
\begin{aligned}
\mathcal{T}^{\prime}(u) & =\mathrm{L}_{1}^{\prime}\left(u-\xi_{1}\right) \mathrm{L}_{2}^{\prime}\left(u-\xi_{2}\right) \ldots \mathrm{L}_{N}^{\prime}\left(u-\xi_{N}\right) \\
& =M_{l}^{-1}(u) \mathcal{T}(u) M_{N+l}(u)=\left(\begin{array}{cc}
A^{l}(u) & B^{l}(u) \\
C^{l}(u) & D^{l}(u)
\end{array}\right) .
\end{aligned}
$$

The global vacuum vectors are defined as

$$
\left|\Omega^{l}\right\rangle=\left|\omega_{1}^{l}\right\rangle \otimes\left|\omega_{2}^{l}\right\rangle \otimes \ldots \otimes\left|\omega_{N}^{l}\right\rangle .
$$


According to (2.43), (2.44), the action of the operators $A^{l}(u), D^{l}(u)$ and $C^{l}(u)$ on the global vacuum vector is given by

$$
\begin{aligned}
& C^{l}(u)\left|\Omega^{l}\right\rangle=0, \\
& A^{l}(u)\left|\Omega^{l}\right\rangle=\prod_{i=1}^{N} \theta_{1}\left(u-\xi_{i}+\eta \mid \tau\right)\left|\Omega^{l+1}\right\rangle, \\
& D^{l}(u)\left|\Omega^{l}\right\rangle=\prod_{i=1}^{N} \theta_{1}\left(u-\xi_{i} \mid \tau\right)\left|\Omega^{l-1}\right\rangle .
\end{aligned}
$$

The same formulas $(2.24),(2.25)$ allow one to check that the local dual vacuum vector

$$
\left\langle\bar{\omega}_{k}^{l}\right|=\left\langle\phi^{\perp}\left(t_{k+l}-\xi_{k}\right)\right|
$$

satisfies the following properties:

$$
\begin{aligned}
& \left\langle\bar{\omega}_{k}^{l}\right| \mathrm{b}_{k}^{\prime}=0, \\
& \left\langle\bar{\omega}_{k}^{l}\right| \mathrm{a}_{k}^{\prime}=\gamma_{k+l-1} \gamma_{k+l}^{-1} \theta_{1}\left(u-\xi_{k}+\eta \mid \tau\right)\left\langle\bar{\omega}_{k}^{l-1}\right|, \\
& \left\langle\bar{\omega}_{k}^{l}\right| \mathrm{d}_{k}^{\prime}=\gamma_{k+l} \gamma_{k+l-1}^{-1} \theta_{1}\left(u-\xi_{k} \mid \tau\right)\left\langle\bar{\omega}_{k}^{l+1}\right| .
\end{aligned}
$$

The global dual (left) vacuum vectors are defined as tensor products of the local ones:

$$
\left\langle\bar{\Omega}^{l}\right|=\left\langle\bar{\omega}_{1}^{l}\right| \otimes\left\langle\bar{\omega}_{2}^{l}\right| \otimes \ldots \otimes\left\langle\bar{\omega}_{N}^{l}\right| .
$$

The action of the operators $A^{l}(u), D^{l}(u), B^{l}(u)$ to the left vacuum is given by

$$
\begin{aligned}
& \left\langle\bar{\Omega}^{l}\right| B^{l}(u)=0, \\
& \left\langle\bar{\Omega}^{l}\right| A^{l}(u)=\gamma_{l} \gamma_{l+N}^{-1} \prod_{i=1}^{N} \theta_{1}\left(u-\xi_{i}+\eta \mid \tau\right)\left\langle\bar{\Omega}^{l-1}\right|, \\
& \left\langle\bar{\Omega}^{l}\right| D^{l}(u)=\gamma_{l+N} \gamma_{l}^{-1} \prod_{i=1}^{N} \theta_{1}\left(u-\xi_{i} \mid \tau\right)\left\langle\bar{\Omega}^{l+1}\right| .
\end{aligned}
$$

These formulas will be used in the generalized algebraic Bethe ansatz.

\section{The generalized algebraic Bethe ansatz}

In this section, we construct off-shell and on-shell Bethe vectors and describe their properties. We continue to use the "bra-ket" notation in order to distinguish between right $\left|\Psi_{\nu}\left(u_{1}, \ldots, u_{n}\right)\right\rangle$ and left (dual) $\left\langle\Psi_{\nu}\left(v_{1}, \ldots, v_{n}\right)\right|$ Bethe vectors. We draw the reader's attention to the fact that the meaning of this notation is completely different from that in the previous section. First of all, in distinction of the two-component vectors $|\phi(u)\rangle$ and $\left\langle\phi^{\perp}(u)\right|$ the Bethe vectors $\left|\Psi_{\nu}\left(u_{1}, \ldots, u_{n}\right)\right\rangle$ belong to the quantum space of the model, that is, $\left(\mathbb{C}^{2}\right)^{\otimes N}$. Respectively, the dual vectors $\left\langle\Psi_{\nu}\left(v_{1}, \ldots, v_{n}\right)\right|$ belong to the dual space. Besides, since the procedures for constructing left and right vectors are slightly different, 
we generally do not require that the left and right vectors be connected by transposition or Hermitian conjugation. In particular, the scalar product $\left\langle\Psi_{\nu}\left(v_{1}, \ldots, v_{n}\right) \mid \Psi_{\nu}\left(v_{1}, \ldots, v_{n}\right)\right\rangle$, generally speaking, is not the square of the vector norm. However, it does become the square of the norm for the values of parameters that ensure the positivity of Boltzmann weights (2.6) or the self-conjugacy of the Hamiltonian (2.20). This treatment of the right and left Bethe vectors is traditional in the algebraic Bethe ansatz approach.

\subsection{The permutation relations}

Let us introduce the generalized (gauge-transformed) monodromy matrices

$$
\mathcal{T}_{k, l}(u)=M_{k}^{-1}(u) \mathcal{T}(u) M_{l}(u)=\left(\begin{array}{cc}
A_{k, l}(u) & B_{k, l}(u) \\
C_{k, l}(u) & D_{k, l}(u)
\end{array}\right) .
$$

Note that in this new notation $\mathcal{T}^{\prime}(u)=\mathcal{T}_{l, l+N}(u)$. We have:

$$
\begin{aligned}
A_{k, l}(u) & =\frac{\left\langle\phi^{\perp}\left(t_{k}-u\right)|\mathcal{T}(u)| \phi\left(s_{l}+u\right)\right\rangle}{\left\langle\phi^{\perp}\left(t_{k}-u\right) \mid \phi\left(s_{k}+u\right)\right\rangle}, \\
B_{k, l}(u) & =\gamma_{l} \frac{\left\langle\phi^{\perp}\left(t_{k}-u\right)|\mathcal{T}(u)| \phi\left(t_{l}-u\right)\right\rangle}{\left\langle\phi^{\perp}\left(t_{k}-u\right) \mid \phi\left(s_{k}+u\right)\right\rangle}, \\
C_{k, l}(u) & =-\frac{1}{\gamma_{k}} \frac{\left\langle\phi^{\perp}\left(s_{k}+u\right)|\mathcal{T}(u)| \phi\left(s_{l}+u\right)\right\rangle}{\left\langle\phi^{\perp}\left(t_{k}-u\right) \mid \phi\left(s_{k}+u\right)\right\rangle}, \\
D_{k, l}(u) & =-\frac{\gamma_{l}}{\gamma_{k}} \frac{\left\langle\phi^{\perp}\left(s_{k}+u\right)|\mathcal{T}(u)| \phi\left(t_{l}-u\right)\right\rangle}{\left\langle\phi^{\perp}\left(t_{k}-u\right) \mid \phi\left(s_{k}+u\right)\right\rangle} .
\end{aligned}
$$

It follows from equations (2.14) that the generalized monodromy matrix has the following quasiperiodicity properties:

$$
\begin{aligned}
\mathcal{T}_{k, l}(u+1) & =\mathcal{T}_{k, l}(u), \\
\mathcal{T}_{k, l}(u+\tau) & =e^{-\pi i c(u)}\left(\begin{array}{cc}
e^{\pi i s_{k}} & 0 \\
0 & -e^{-\pi i t_{k}}
\end{array}\right) \mathcal{T}_{k, l}(u)\left(\begin{array}{cc}
e^{-\pi i s_{l}} & 0 \\
0 & -e^{\pi i t_{l}}
\end{array}\right) \\
& =e^{-\pi i c(u)}\left(\begin{array}{cc}
e^{\pi i\left(s_{k}-s_{l}\right)} A_{k, l}(u) & -e^{\pi i\left(s_{k}+t_{l}\right)} B_{k, l}(u) \\
-e^{-\pi i\left(s_{l}+t_{k}\right)} C_{k, l}(u) & e^{\pi i\left(t_{l}-t_{k}\right)} D_{k, l}(u)
\end{array}\right),
\end{aligned}
$$

where $c(u)$ is defined in (2.15).

For the calculations below it is convenient to introduce a temporary notation for the vectors and covectors

$$
\begin{aligned}
X^{l}(u) & =\left|\phi\left(s_{l}+u\right)\right\rangle, & Y^{l}(u) & =\left|\phi\left(t_{l}-u\right)\right\rangle, \\
\tilde{X}^{k}(u) & =\left\langle\phi^{\perp}\left(s_{k}+u\right)\right|, & \tilde{Y}^{k}(u) & =\left\langle\phi^{\perp}\left(t_{k}-u\right)\right|,
\end{aligned}
$$

then

$$
\begin{array}{ll}
A_{k, l}(u)=-\frac{\gamma_{k}}{\mu(u)} \tilde{Y}^{k}(u) \mathcal{T}(u) X^{l}(u), & B_{k, l}(u)=-\frac{\gamma_{k} \gamma_{l}}{\mu(u)} \tilde{Y}^{k}(u) \mathcal{T}(u) Y^{l}(u), \\
C_{k, l}(u)=\frac{1}{\mu(u)} \tilde{X}^{k}(u) \mathcal{T}(u) X^{l}(u), & D_{k, l}(u)=\frac{\gamma_{l}}{\mu(u)} \tilde{X}^{k}(u) \mathcal{T}(u) Y^{l}(u) .
\end{array}
$$


In this notation, equations $(2.24),(2.25),(2.32),(2.33)$ look as follows:

$$
\begin{aligned}
\mathrm{R}_{12}(u-v) X_{1}^{l+1}(u) X_{2}^{l}(v) & =\theta_{1}(u-v+\eta \mid \tau) X_{1}^{l}(u) X_{2}^{l+1}(v), \\
\mathrm{R}_{12}(u-v) Y_{1}^{l-1}(u) Y_{2}^{l}(v) & =\theta_{1}(u-v+\eta \mid \tau) Y_{1}^{l}(u) Y_{2}^{l-1}(v), \\
\mathrm{R}_{12}(u-v) Y_{1}^{l+1}(u) X_{2}^{l}(v) & =f_{l}^{+}(u-v) Y_{1}^{l}(u) X_{2}^{l-1}(v)+g_{l}(v-u) X_{1}^{l}(u) Y_{2}^{l+1}(v), \\
\mathrm{R}_{12}(u-v) X_{1}^{k}(u) Y_{2}^{k-1}(v) & =f_{k}^{-}(u-v) X_{1}^{k+1}(u) Y_{2}^{k}(v)+g_{k}(u-v) Y_{1}^{k-1}(u) X_{2}^{k}(v), \\
\mathrm{R}_{12}(u-v) Y_{1}^{l}(u) X_{2}^{l+1}(v) & =f_{l}^{+}(u-v) Y_{1}^{l-1}(u) X_{2}^{l}(v)+g_{l}(v-u) X_{1}^{l+1}(u) Y_{2}^{l}(v), \\
\mathrm{R}_{12}(u-v) X_{1}^{k-1}(u) Y_{2}^{k}(v) & =f_{k}^{-}(u-v) X_{1}^{k}(u) Y_{2}^{k+1}(v)+g_{k}(u-v) Y_{1}^{k}(u) X_{2}^{k-1}(v),
\end{aligned}
$$

where

$$
f_{k}^{ \pm}(u)=\frac{\theta_{1}(u \mid \tau) \theta_{2}\left(\tau_{k \pm 1} \mid \tau\right)}{\theta_{2}\left(\tau_{k} \mid \tau\right)}, \quad g_{k}(u)=\frac{\theta_{1}(\eta \mid \tau) \theta_{2}\left(\tau_{k}+u \mid \tau\right)}{\theta_{2}\left(\tau_{k} \mid \tau\right)} .
$$

Similar relations hold for the covectors $\tilde{X}^{l}(u), \tilde{Y}^{l}(u)$; they are obtained by transposition in both spaces.

Multiplying both sides of the $R T T=T T R$ relation (2.16) by the vectors $Y_{1}^{l-1}(u) Y_{2}^{l}(v)$ from the right and $\tilde{Y}_{1}^{k-1}(u) \tilde{Y}_{2}^{k}(v)$ from the left and using (3.5), one obtains the permutation relation

$$
B_{k+1, l}(u) B_{k, l+1}(v)=B_{k+1, l}(v) B_{k, l+1}(u) .
$$

Similarly, multiplying both sides of $(2.16)$ by the vectors $X_{1}^{l+1}(u) X_{2}^{l}(v)$ from the right and $\tilde{X}_{1}^{k+1}(u) \tilde{X}_{2}^{k}(v)$ from the left and using (3.4), we get

$$
C_{k, l+1}(u) C_{k+1, l}(v)=C_{k, l+1}(v) C_{k+1, l}(u) .
$$

The commutation relations between $A$ - and $B$-operators are obtained by multiplying both sides of (2.16) by the vectors $Y_{1}^{l+1}(u) X_{2}^{l}(v)$ from the right and $\tilde{Y}_{1}^{k-1}(u) \tilde{Y}_{2}^{k}(v)$ from the left and using the transposed version of (3.5) and (3.6):

$$
\begin{aligned}
& \theta_{1}(u-v+\eta \mid \tau) B_{k, l+1}(u) A_{k-1, l}(v) \\
& \quad=\theta_{1}(u-v \mid \tau) A_{k, l-1}(v) B_{k-1, l}(u)+g_{l}(v-u) B_{k, l+1}(v) A_{k-1, l}(u) .
\end{aligned}
$$

The other commutation relations can be obtained in a similar way. Multiplying both sides of (2.16) by the vectors $Y_{1}^{l}(u) Y_{2}^{l+1}(v)$ from the right and $\tilde{X}_{1}^{k}(u) \tilde{Y}_{2}^{k-1}(v)$ from the left and using the transposed version of (3.7), one obtains

$$
\begin{aligned}
\theta_{1}(u-v+\eta \mid \tau) & B_{k-1, l}(v) D_{k, l+1}(u) \\
& =\theta_{1}(u-v \mid \tau) D_{k+1, l}(u) B_{k, l+1}(v)+g_{k}(u-v) B_{k-1, l}(u) D_{k, l+1}(v) .
\end{aligned}
$$

Multiplying both sides of the $R T T=T T R$ relation by the vectors $X_{1}^{l+1}(u) X_{2}^{l}(v)$ from the right and $\tilde{X}_{1}^{k-1}(u) \tilde{Y}_{2}^{k}(v)$ from the left and using the transposed version of (3.9), one 
obtains:

$$
\begin{aligned}
\theta_{1}(u-v & +\eta \mid \tau) A_{k, l+1}(v) C_{k-1, l}(u) \\
& =\frac{\gamma_{k}^{2}}{\gamma_{k+1} \gamma_{k-1}} \theta_{1}(u-v \mid \tau) C_{k, l+1}(u) A_{k+1, l}(v)+g_{k}(u-v) A_{k, l+1}(u) C_{k-1, l}(v) .
\end{aligned}
$$

Finally, multiplying both sides of the $R T T=T T R$ relation by the vectors $Y_{1}^{l}(u) X_{2}^{l+1}(v)$ from the right and $\tilde{X}_{1}^{k+1}(u) \tilde{X}_{2}^{k}(v)$ from the left and using (3.8), one obtains:

$$
\begin{aligned}
\theta_{1}(u-v & +\eta \mid \tau) D_{k, l}(u) C_{k+1, l+1}(v) \\
& =\frac{\gamma_{l}^{2}}{\gamma_{l+1} \gamma_{l-1}} \theta_{1}(u-v \mid \tau) C_{k, l}(v) D_{k+1, l-1}(u)+g_{l}(v-u) D_{k, l}(v) C_{k+1, l+1}(u) .
\end{aligned}
$$

These are the main operator permutation relations used in the generalized algebraic Bethe ansatz procedure.

\subsection{Right eigenvectors}

Let us consider a vector

$$
\left|\Psi^{l}\left(u_{1}, \ldots, u_{n}\right)\right\rangle=B_{l-1, l+1}\left(u_{1}\right) B_{l-2, l+2}\left(u_{2}\right) \ldots B_{l-n, l+n}\left(u_{n}\right)\left|\Omega^{l-n}\right\rangle .
$$

We recall that $n$ is fixed and is equal to $N / 2$. The commutation relation (3.10) implies that this vector is a symmetric function of the parameters $u_{1}, \ldots, u_{n}$. We are going to act to this vector by the transfer matrix $\mathrm{T}(u)=A_{l, l}(u)+D_{l, l}(u)$. To this end, let us rewrite equations (3.12), (3.13) in a more convenient form suitable for moving the operators $A$ and $D$ to the right through $B$ :

$$
\begin{aligned}
& A_{k, l}(u) B_{k-1, l+1}(v) \\
& \quad=\alpha(u-v) B_{k, l+2}(v) A_{k-1, l+1}(u)+\beta_{l+1}(u-v) B_{k, l+2}(u) A_{k-1, l+1}(v) \\
& \quad \begin{array}{r}
D_{k, l}(u) B_{k-1, l+1}(v) \\
\quad \\
\quad \alpha(v-u) B_{k-2, l}(v) D_{k-1, l+1}(u)-\beta_{k-1}(u-v) B_{k-2, l}(u) D_{k-1, l+1}(v),
\end{array}
\end{aligned}
$$

where

$$
\alpha(u)=\frac{\theta_{1}(u-\eta \mid \tau)}{\theta_{1}(u \mid \tau)}, \quad \beta_{k}(u)=\frac{\theta_{1}(\eta \mid \tau) \theta_{2}\left(\tau_{k}+u \mid \tau\right)}{\theta_{1}(u \mid \tau) \theta_{2}\left(\tau_{k} \mid \tau\right)} .
$$

The action of the operators $A_{l, l}(u), D_{l, l}(u)$ to the vector (3.16) can be found, with the help of the standard algebraic Bethe ansatz argument, using the permutation relations (3.17), (3.18) and the property (2.45). The result is:

$$
\begin{aligned}
A_{l, l}(u)\left|\Psi^{l}\left(u_{1}, \ldots, u_{n}\right)\right\rangle= & T_{A}(u)\left|\Psi^{l+1}\left(u_{1}, \ldots, u_{n}\right)\right\rangle \\
& +\sum_{j=1}^{n} \Lambda_{A, j}^{l}(u)\left|\Psi^{l+1}\left(u_{1}, \ldots, u_{j-1}, u, u_{j+1}, \ldots, u_{n}\right)\right\rangle, \\
D_{l, l}(u)\left|\Psi^{l}\left(u_{1}, \ldots, u_{n}\right)\right\rangle= & T_{D}(u)\left|\Psi^{l-1}\left(u_{1}, \ldots, u_{n}\right)\right\rangle \\
& +\sum_{j=1}^{n} \Lambda_{D, j}^{l}(u)\left|\Psi^{l-1}\left(u_{1}, \ldots, u_{j-1}, u, u_{j+1}, \ldots, u_{n}\right)\right\rangle,
\end{aligned}
$$


where

$$
\begin{aligned}
T_{A}(u) & =\prod_{i=1}^{N} \theta_{1}\left(u-\xi_{i}+\eta \mid \tau\right) \prod_{k=1}^{n} \frac{\theta_{1}\left(u-u_{k}-\eta \mid \tau\right)}{\theta_{1}\left(u-u_{k} \mid \tau\right)} \\
T_{D}(u) & =\prod_{i=1}^{N} \theta_{1}\left(u-\xi_{i} \mid \tau\right) \prod_{k=1}^{n} \frac{\theta_{1}\left(u-u_{k}+\eta \mid \tau\right)}{\theta_{1}\left(u-u_{k} \mid \tau\right)} \\
\Lambda_{A, j}^{l}(u) & =\frac{\theta_{1}(\eta \mid \tau)}{\theta_{1}^{\prime}(0 \mid \tau)} \Phi\left(u-u_{j}, \tau_{l+1}+\frac{1}{2}\right) \prod_{i=1}^{N} \theta_{1}\left(u_{j}-\xi_{i}+\eta \mid \tau\right) \prod_{k=1, \neq j}^{n} \frac{\theta_{1}\left(u_{j}-u_{k}-\eta \mid \tau\right)}{\theta_{1}\left(u_{j}-u_{k} \mid \tau\right)} \\
\Lambda_{D, j}^{l}(u) & =-\frac{\theta_{1}(\eta \mid \tau)}{\theta_{1}^{\prime}(0 \mid \tau)} \Phi\left(u-u_{j}, \tau_{l-1}+\frac{1}{2}\right) \prod_{i=1}^{N} \theta_{1}\left(u_{j}-\xi_{i} \mid \tau\right) \prod_{k=1, \neq j}^{n} \frac{\theta_{1}\left(u_{j}-u_{k}+\eta \mid \tau\right)}{\theta_{1}\left(u_{j}-u_{k} \mid \tau\right)}
\end{aligned}
$$

In the last two formulas we have introduced a function

$$
\Phi(u, v)=\frac{\theta_{1}^{\prime}(0 \mid \tau) \theta_{1}(u+v \mid \tau)}{\theta_{1}(u \mid \tau) \theta_{1}(v \mid \tau)} .
$$

It has a simple pole at $u=0$ with the residue 1 .

Consider now the Fourier transform of the vector $\left|\Psi^{l}\right\rangle$ :

$$
\left|\Psi_{\nu}\left(u_{1}, \ldots, u_{n}\right)\right\rangle=\sum_{l \in \mathbb{Z}} e^{-i l \pi \eta \nu}\left|\Psi^{l}\left(u_{1}, \ldots, u_{n}\right)\right\rangle .
$$

For arbitrary parameters $u_{j}$ we call such vectors off-shell Bethe vectors. The action of the transfer matrix $\mathrm{T}(u)=A_{l, l}(u)+D_{l, l}(u)$ on such vector is given by

$$
\begin{aligned}
\mathrm{T}(u)\left|\Psi_{\nu}\left(u_{1}, \ldots, u_{n}\right)\right\rangle= & T_{\nu}(u)\left|\Psi_{\nu}\left(u_{1}, \ldots, u_{n}\right)\right\rangle \\
& +\sum_{l \in \mathbb{Z}} \sum_{j=1}^{n} e^{-i l \pi \eta \nu}\left(e^{i \pi \eta \nu} \Lambda_{A, j}^{l-1}(u)+e^{-i \pi \eta \nu} \Lambda_{D, j}^{l+1}(u)\right) \\
& \times\left|\Psi^{l}\left(u_{1}, \ldots, u_{j-1}, u, u_{j+1}, \ldots, u_{n}\right)\right\rangle,
\end{aligned}
$$

where

$$
\begin{aligned}
T_{\nu}(u)= & e^{i \pi \eta \nu} \prod_{i=1}^{N} \theta_{1}\left(u-\xi_{i}+\eta \mid \tau\right) \prod_{k=1}^{n} \frac{\theta_{1}\left(u-u_{k}-\eta \mid \tau\right)}{\theta_{1}\left(u-u_{k} \mid \tau\right)} \\
& +e^{-i \pi \eta \nu} \prod_{i=1}^{N} \theta_{1}\left(u-\xi_{i} \mid \tau\right) \prod_{k=1}^{n} \frac{\theta_{1}\left(u-u_{k}+\eta \mid \tau\right)}{\theta_{1}\left(u-u_{k} \mid \tau\right)} .
\end{aligned}
$$

Note that one can rewrite (3.22) in the form

$$
\begin{aligned}
\mathrm{T}(u)\left|\Psi_{\nu}\left(u_{1}, \ldots, u_{n}\right)\right\rangle= & T_{\nu}(u)\left|\Psi_{\nu}\left(u_{1}, \ldots, u_{n}\right)\right\rangle \\
& -\sum_{l \in \mathbb{Z}} \sum_{j=1}^{n} e^{-i l \pi \eta \nu} \Phi\left(u-u_{j}, \tau_{l}+\frac{1}{2}\right)\left(\underset{u=u_{j}}{\operatorname{res}} T_{\nu}(u)\right) \\
& \times\left|\Psi^{l}\left(u_{1}, \ldots, u_{j-1}, u, u_{j+1}, \ldots, u_{n}\right)\right\rangle .
\end{aligned}
$$

In this form, it is clear that the r.h.s. is regular at $u=u_{j}$ as it should be. 
The eigenvalue of the transfer matrix should be a regular function of $u_{j}$. The conditions $\underset{u=u_{j}}{\operatorname{res}} T_{\nu}(u)=0$ are simultaneously the conditions of cancellation of the "unwanted terms" in (3.24). These conditions have the form of the Bethe equations

$$
e^{2 i \pi \eta \nu} \prod_{i=1}^{N} \frac{\theta_{1}\left(u_{j}-\xi_{i}+\eta \mid \tau\right)}{\theta_{1}\left(u_{j}-\xi_{i} \mid \tau\right)}=\prod_{k=1, \neq j}^{n} \frac{\theta_{1}\left(u_{j}-u_{k}+\eta \mid \tau\right)}{\theta_{1}\left(u_{j}-u_{k}-\eta \mid \tau\right)}
$$

For $N=2$ there is only one Bethe equation and it can be solved explicitly (see appendix B). If the Bethe equations are satisfied, then the unwanted terms cancel and the vector $\left|\Psi_{\nu}\right\rangle=\left|\Psi_{\nu}\left(u_{1}, \ldots, u_{n}\right)\right\rangle$ is an eigenvector of the transfer matrix provided that $\nu$ is such that the series (3.21) converges and is non-zero. Presumably, this holds for some particular values of $\nu$ and $\nu=0$ is among them. We call such vectors on-shell Bethe vectors.

For what follows we need analytical properties of the Bethe vectors as functions of the parameters $u_{j}$. First of all, we note that $\left|\Psi^{l}\right\rangle$ (and, therefore, $\left|\Psi_{\nu}\right\rangle$ is an entire function of $u_{j}$. Indeed, a possible pole could only occur at $u_{j}=(t-s) / 2$ when $\mu(u)$ in the denominator of the expression for $B_{l-j, l+j}\left(u_{j}\right)$ vanishes. In this case the matrix $M_{k}\left(u_{j}\right)$ becomes degenerate and one can immediately see that

$$
\underset{u_{j}=(t-s) / 2}{\operatorname{res}} B_{l-j, l+j}\left(u_{j}\right) \propto \underset{u_{j}=(t-s) / 2}{\operatorname{res}} C_{l-j, l+j}\left(u_{j}\right) .
$$

Using the fact that the Bethe vector is a symmetric function of the $u_{i}$ 's, one can move $u_{j}$ to the very right end of the chain of the $B$-operators, where we have

$$
\underset{u_{j}=(t-s) / 2}{\operatorname{res}} B_{l-n, l+n}\left(u_{j}\right)\left|\Omega^{l-n}\right\rangle=\underset{u_{j}=(t-s) / 2}{\operatorname{res}} C_{l-n, l+n}\left(u_{j}\right)\left|\Omega^{l-n}\right\rangle=0 .
$$

Therefore, $\operatorname{res}_{u_{j}=(t-s) / 2}\left|\Psi^{l}\left(u_{1}, \ldots, u_{n}\right)\right\rangle=0$ and thus the Bethe vector is a regular function of each of $u_{j}$.

Next, let us derive the quasiperiodic properties of the Bethe vector under the shifts $u_{j} \rightarrow u_{j}+1$ and $u_{j} \rightarrow u_{j}+\tau$. Using (3.3), we have:

$$
\begin{aligned}
& B_{l-j, l+j}(u+1)=B_{l-j, l+j}(u), \\
& B_{l-j, l+j}(u+\tau)=-e^{\pi i(s+t)+2 \pi i l \eta-\pi i c(u)} B_{l-j, l+j}(u),
\end{aligned}
$$

where $c(u)$ is given by $(2.15)$. It then follows that

$$
\begin{aligned}
& \left|\Psi_{\nu}\left(u_{1}, \ldots, u_{j-1}, u_{j}+1, u_{j+1}, \ldots, u_{n}\right)\right\rangle=\left|\Psi_{\nu}\left(u_{1}, \ldots, u_{n}\right)\right\rangle \\
& \left|\Psi_{\nu}\left(u_{1}, \ldots, u_{j-1}, u_{j}+\tau, u_{j+1}, \ldots, u_{n}\right)\right\rangle=-e^{\pi i(s+t)-\pi i c(u)}\left|\Psi_{\nu-2}\left(u_{1}, \ldots, u_{n}\right)\right\rangle .
\end{aligned}
$$

In particular, if the vector is on-shell and $\left\{\nu, u_{1}, \ldots, u_{n}\right\}$ is the corresponding solution of the Bethe equations, the set $\left\{\nu+2, u_{1}, \ldots, u_{j}+\tau, \ldots, u_{n}\right\}$ also solves the Bethe equations and the two eigenvectors are proportional to each other, i.e., correspond to the same physical state. This fact was mentioned in [13]. 


\subsection{Left eigenvectors}

For the construction of left eigenvectors of the transfer matrix it is convenient to redefine the operators $A_{k l}, B_{k, l}, C_{k l}, D_{k l}$ in the following way:

$$
\bar{A}_{k l}=\gamma_{k}^{-1} \gamma_{l} A_{k l}, \quad \bar{B}_{k l}=\gamma_{k}^{-1} \gamma_{l}^{-1} B_{k l}, \quad \bar{C}_{k l}=\gamma_{k} \gamma_{l} C_{k l}, \quad \bar{D}_{k l}=\gamma_{k} \gamma_{l}^{-1} D_{k l} .
$$

Note that these operators act to the left vacuum as follows (see (2.49)):

$$
\begin{aligned}
\left\langle\bar{\Omega}^{l}\right| \bar{A}_{l, l+N}(u) & =\prod_{i=1}^{N} \theta_{1}\left(u-\xi_{i}+\eta \mid \tau\right)\left\langle\bar{\Omega}^{l-1}\right|, \\
\left\langle\bar{\Omega}^{l}\right| \bar{D}_{l, l+N}(u) & =\prod_{i=1}^{N} \theta_{1}\left(u-\xi_{i} \mid \tau\right)\left\langle\bar{\Omega}^{l+1}\right| .
\end{aligned}
$$

The new operators are matrix elements of the gauge-transformed quantum monodromy matrix $\overline{\mathcal{T}}(u)=\bar{M}_{k}^{-1}(u) \mathcal{T}(u) \bar{M}_{l}(u)$ by means of the matrix

$$
\bar{M}_{k}(u)=\left(\begin{array}{c}
\gamma_{k} \theta_{1}\left(s_{k}+u \mid 2 \tau\right) \theta_{1}\left(t_{k}-u \mid 2 \tau\right) \\
\gamma_{k} \theta_{4}\left(s_{k}+u \mid 2 \tau\right) \theta_{4}\left(t_{k}-u \mid 2 \tau\right)
\end{array}\right)
$$

(comparing to the matrix (2.38), the first rather than second column is multiplied by $\gamma_{k}$ ).

Let us consider the dual vector

$$
\left\langle\Psi^{l}\left(v_{1}, \ldots, v_{n}\right)\right|=\left\langle\bar{\Omega}^{l-n}\right| \bar{C}_{l-n, l+n}\left(v_{n}\right) \ldots \bar{C}_{l-2, l+2}\left(v_{2}\right) \bar{C}_{l-1, l+1}\left(v_{1}\right) .
$$

The commutation relation (3.11) implies that this vector is a symmetric function of the parameters $v_{1}, \ldots, v_{n}$. We are going to act to this vector by the transfer matrix $\mathrm{T}(u)=$ $\bar{A}_{l, l}(u)+\bar{D}_{l, l}(u)$ to the left. To this end, let us rewrite equations (3.14), (3.15) in a more convenient form suitable for moving the operators $\bar{A}$ and $\bar{D}$ to the left through $\bar{C}$ :

$$
\begin{aligned}
& \bar{C}_{k-1, l+1}(v) \bar{A}_{k, l}(u) \\
& \quad=\alpha(u-v) \bar{A}_{k-1, l+1}(u) \bar{C}_{k-2, l}(v)-\beta_{k-1}(v-u) \bar{A}_{k-1, l+1}(v) \bar{C}_{k-2, l}(u), \\
& \quad \bar{C}_{k-1, l+1}(v) \bar{D}_{k, l}(u) \\
& \quad=\alpha(v-u) \bar{D}_{k-1, l+1}(u) \bar{C}_{k, l+2}(v)+\beta_{l+1}(v-u) \bar{D}_{k-1, l+1}(v) \bar{C}_{k, l+2}(u),
\end{aligned}
$$

with the same functions $\alpha(u), \beta_{k}(u)$ as in (3.19).

Consider now the Fourier transform of the dual vector $\left\langle\Psi^{l}\right|$ :

$$
\left\langle\Psi_{\nu}\left(v_{1}, \ldots, v_{n}\right)\right|=\sum_{l \in \mathbb{Z}} e^{i l \pi \eta \nu}\left\langle\Psi^{l}\left(v_{1}, \ldots, v_{n}\right)\right| .
$$

The arguments similar to the ones used in the case of right vectors lead to the following formula for the action of the transfer matrix to the dual vector:

$$
\begin{aligned}
\left\langle\Psi_{\nu}\left(v_{1}, \ldots, v_{n}\right)\right| \mathrm{T}(u)= & T_{\nu}(u)\left\langle\Psi_{\nu}\left(v_{1}, \ldots, v_{n}\right)\right| \\
& +\sum_{l \in \mathbb{Z}} \sum_{j=1}^{n} e^{i l \pi \eta \nu} \Phi\left(v_{j}-u, \tau_{l}+\frac{1}{2}\right)\left(\underset{u=v_{j}}{\operatorname{res}} T_{\nu}(u)\right) \\
& \times\left\langle\Psi^{l}\left(v_{1}, \ldots, v_{j-1}, u, v_{j+1}, \ldots, v_{n}\right)\right| .
\end{aligned}
$$


Again, the conditions $\underset{u=v_{j}}{\operatorname{res}} T_{\nu}(u)=0$ for all $j$ ensure cancellation of the "unwanted terms" in (3.34) and are equivalent to the Bethe equations (3.25) for the parameters $v_{j}$.

Analytic properties of the left Bethe vectors are similar to those of the right ones. They are regular functions of the parameters $v_{i}$. Using (3.3), we have:

$$
\begin{aligned}
& \bar{C}_{l-j, l+j}(u+1)=\bar{C}_{l-j, l+j}(u), \\
& \bar{C}_{l-j, l+j}(u+\tau)=-e^{-\pi i(s+t)-2 \pi i l \eta-\pi i c(u)} \bar{C}_{l-j, l+j}(u) .
\end{aligned}
$$

It then follows that

$$
\begin{aligned}
\left\langle\Psi_{\nu}\left(v_{1}, \ldots, v_{j-1}, v_{j}+1, v_{j+1}, \ldots, v_{n}\right)\right| & =\left\langle\Psi_{\nu}\left(v_{1}, \ldots, v_{n}\right)\right| \\
\left\langle\Psi_{\nu}\left(v_{1}, \ldots, v_{j-1}, v_{j}+\tau, v_{j+1}, \ldots, v_{n}\right)\right| & =-e^{-\pi i(s+t)-\pi i c\left(v_{j}\right)}\left\langle\Psi_{\nu-2}\left(v_{1}, \ldots, v_{n}\right)\right| .
\end{aligned}
$$

\subsection{Action of the operators $U_{a}$ to Bethe vectors}

The key identity necessary to derive how the operators $\mathrm{U}_{a}$ act to (in general off-shell) Bethe vectors is

$$
\sigma_{a} \otimes \mathrm{U}_{a} \mathcal{T}(u)=\mathcal{T}(u) \sigma_{a} \otimes \mathrm{U}_{a},
$$

which follows from (2.9) (here $\sigma_{a}$ acts in the auxiliary space $\mathbb{C}^{2}$ and $\mathbf{U}_{a}$ acts in the quantum space $\mathcal{H}$ ), or, in more detail:

$$
\begin{aligned}
& \left(\begin{array}{ll}
A(u) \mathrm{U}_{1} & B(u) \mathrm{U}_{1} \\
C(u) \mathrm{U}_{1} & D(u) \mathrm{U}_{1}
\end{array}\right)=\left(\begin{array}{ll}
\mathrm{U}_{1} D(u) & \mathrm{U}_{1} C(u) \\
\mathrm{U}_{1} B(u) & \mathrm{U}_{1} A(u)
\end{array}\right), \\
& \left(\begin{array}{ll}
A(u) \mathrm{U}_{3} & B(u) \mathrm{U}_{3} \\
C(u) \mathrm{U}_{3} & D(u) \mathrm{U}_{3}
\end{array}\right)=\left(\begin{array}{rr}
\mathrm{U}_{3} A(u) & -\mathrm{U}_{3} B(u) \\
-\mathrm{U}_{3} C(u) & \mathrm{U}_{3} D(u)
\end{array}\right) .
\end{aligned}
$$

It then follows that

$$
\begin{aligned}
& \mathrm{U}_{1} B_{k, l}(u ; s, t)=e^{-\pi i(2 u+(k+l) \eta+2 s+\tau)} B_{k, l}(u ; s+\tau, t+\tau) \mathrm{U}_{1}, \\
& \bar{C}_{k, l}(u ; s, t) \mathrm{U}_{1}=e^{-\pi i(-2 u+(k+l) \eta+2 t+\tau)} \mathrm{U}_{1} \bar{C}_{k, l}(u ; s+\tau, t+\tau), \\
& \mathrm{U}_{3} B_{k, l}(u ; s, t)=-B_{k, l}(u ; s+1, t+1) \mathrm{U}_{3}, \\
& \bar{C}_{k, l}(u ; s, t) \mathrm{U}_{3}=-\mathrm{U}_{3} \bar{C}_{k, l}(u ; s+1, t+1),
\end{aligned}
$$

and also

$$
\begin{aligned}
& \mathrm{U}_{1} B_{k, l}(u ; s, t)=-e^{-\pi i\left(t_{k}+t_{l}+s-t\right)} B_{k, l}(u ; s+\tau, t-\tau) \mathrm{U}_{1}, \\
& \bar{C}_{k, l}(u ; s, t) \mathrm{U}_{1}=-e^{\pi i\left(s_{k}+s_{l}+t-s\right)} \mathrm{U}_{1} \bar{C}_{k, l}(u ; s+\tau, t-\tau), \\
& \mathrm{U}_{3} B_{k, l}(u ; s, t)=B_{k, l}(u ; s+1, t-1) \mathrm{U}_{3}, \\
& \bar{C}_{k, l}(u ; s, t) \mathrm{U}_{3}=\mathrm{U}_{3} \bar{C}_{k, l}(u ; s+1, t-1) .
\end{aligned}
$$


It is also straightforward to find how the operators $\mathrm{U}_{a}$ act to the vacua:

$$
\begin{aligned}
\mathrm{U}_{1}\left|\Omega^{l-n}(s)\right\rangle & =(-1)^{n} e^{\pi i\left(N s_{l}-n \eta+\sum_{k} \xi_{k}+n \tau\right)}\left|\Omega^{l-n}(s+\tau)\right\rangle, \\
\left\langle\bar{\Omega}^{l-n}(t)\right| \mathrm{U}_{1} & =(-1)^{n} e^{\pi i\left(N t_{l}+n \eta-\sum_{k} \xi_{k}+n \tau\right)}\left\langle\bar{\Omega}^{l-n}(t+\tau)\right|, \\
\mathrm{U}_{3}\left|\Omega^{l-n}(s)\right\rangle & =\left|\Omega^{l-n}(s+1)\right\rangle, \\
\left\langle\bar{\Omega}^{l-n}(t)\right| \mathrm{U}_{3} & =\left\langle\bar{\Omega}^{l-n}(t+1)\right| .
\end{aligned}
$$

Combining equations (3.38)-(3.49), one can derive the following properties of the Bethe vectors:

$$
\begin{aligned}
& \mathrm{U}_{1}\left|\Psi_{\mu}\left(u_{1}, \ldots, u_{n} ; s, t\right)\right\rangle=(-1)^{n} e^{-2 \pi i \sigma\left(u_{1}, \ldots, u_{n}\right)}\left|\Psi_{\mu}\left(u_{1}, \ldots, u_{n} ; s+\tau, t+\tau\right)\right\rangle, \\
&\left\langle\Psi_{\nu}\left(v_{1}, \ldots, v_{n} ; s, t\right)\right| \mathrm{U}_{1}=(-1)^{n} e^{2 \pi i \sigma\left(v_{1}, \ldots, v_{n}\right)}\left\langle\Psi_{\nu}\left(v_{1}, \ldots, v_{n} ; s+\tau, t+\tau\right)\right|, \\
& \mathrm{U}_{3}\left|\Psi_{\mu}\left(u_{1}, \ldots, u_{n} ; s, t\right)\right\rangle=(-1)^{n}\left|\Psi_{\mu}\left(u_{1}, \ldots, u_{n} ; s+1, t+1\right)\right\rangle, \\
&\left\langle\Psi_{\nu}\left(v_{1}, \ldots, v_{n} ; s, t\right)\right| \mathrm{U}_{3}=(-1)^{n}\left\langle\Psi_{\nu}\left(v_{1}, \ldots, v_{n} ; s+1, t+1\right)\right|,
\end{aligned}
$$

where

$$
\sigma\left(v_{1}, \ldots, v_{n}\right)=\sum_{i=1}^{n} v_{i}-\frac{1}{2} \sum_{k=1}^{N} \xi_{k}+\frac{1}{2} n \eta
$$

and

$$
\begin{aligned}
& \mathrm{U}_{1}\left|\Psi^{l}\left(u_{1}, \ldots, u_{n} ; s, t\right)\right\rangle=e^{\pi i n(s-t-\eta)+\pi i n \tau+\pi i \sum_{k} \xi_{k}}\left|\Psi^{l}\left(u_{1}, \ldots, u_{n} ; s+\tau, t-\tau\right)\right\rangle, \\
& \left\langle\Psi^{l}\left(v_{1}, \ldots, v_{n} ; s, t\right)\right| \mathrm{U}_{1}=e^{\pi i n(s-t-\eta)+\pi i n \tau+\pi i \sum_{k} \xi_{k}}\left\langle\Psi^{l}\left(v_{1}, \ldots, v_{n} ; s+\tau, t-\tau\right)\right|, \\
& \mathrm{U}_{3}\left|\Psi^{l}\left(u_{1}, \ldots, u_{n} ; s, t\right)\right\rangle=\left|\Psi^{l}\left(u_{1}, \ldots, u_{n} ; s+1, t-1\right)\right\rangle, \\
& \left\langle\Psi^{l}\left(v_{1}, \ldots, v_{n} ; s, t\right)\right| \mathrm{U}_{3}=\left\langle\Psi^{l}\left(v_{1}, \ldots, v_{n} ; s+1, t-1\right)\right| .
\end{aligned}
$$

\subsection{The case of rational $\eta$}

For irrational values of $\eta$ the Fourier transform (3.21), (3.33) is rather formal because convergence of the infinite series is problematic. Formal expressions of this kind become really meaningful for rational $\eta$,

$$
\eta=\frac{2 P}{Q},
$$

where $P, Q$ are mutually prime integers. In this case all functions in question become $Q$-periodic in $l$ and the infinite Fourier series (3.21) can be substituted by the finite sum

$$
\left|\Psi_{\nu}\left(u_{1}, \ldots, u_{n}\right)\right\rangle=\sum_{l \in \mathbb{Z}_{Q}} e^{-2 \pi i l P \nu / Q}\left|\Psi^{l}\left(u_{1}, \ldots, u_{n}\right)\right\rangle
$$


where $\mathbb{Z}_{Q}=\{0,1, \ldots, Q-1\}$. Because of the $Q$-periodicity the admissible values of $n$ are not restricted by $n=N / 2$ anymore but can be found from the condition

$$
2 n=N(\bmod Q) .
$$

The case $\eta=1 / 2(Q=4)$ is the case of free fermions when the 8 -vertex model splits into two Ising models. In this case the right hand side of Bethe equations becomes $(-1)^{n-1}$, so the Bethe equations convert into $n$ uncoupled equations for each $u_{j}$ separately.

\subsection{Dependence of the eigenvectors on $s, t$}

Here we investigate the dependence of the left eigenvectors $\left\langle\Psi_{\nu}\right|$ on the parameters $s, t$. It is natural to expect that the eigenvectors of the transfer matrix do not essentially depend on these auxiliary parameters, i.e., all the dependence is concentrated in the common scalar factor:

$$
\left\langle\Psi_{\nu}\right|=\varphi_{\nu}(x, y)\left\langle\Psi_{\nu}^{(0)}\right|
$$

where we have denoted

$$
x=\frac{1}{2}(s+t+1), \quad y=\frac{1}{2}(s-t),
$$

and the eigenvector $\left\langle\Psi_{\nu}^{(0)}\right|$ does not depend on $s, t$.

We will use equations (3.51), (3.56), (3.53), (3.58) taking into account the fact that the eigenvectors of the transfer matrix are simultaneously eigenvectors of the operators $\mathrm{U}_{a}$ $(a=1,3)$. Since $\mathrm{U}_{a}$ are involutions, their eigenvalues are $(-1)^{\nu_{a}}$, where $\nu_{a}=0,1$ :

$$
\left\langle\Psi_{\nu}\right| \mathrm{U}_{a}=(-1)^{\nu_{a}}\left\langle\Psi_{\nu}\right| .
$$

Then we have from (3.51), (3.56), (3.53), (3.58):

$$
\begin{aligned}
& \varphi_{\nu}(x+1, y)=(-1)^{n+\nu_{3}} \varphi_{\nu}(x, y), \\
& \varphi_{\nu}(x+\tau, y)=(-1)^{n+\nu_{1}} e^{-2 \pi i \sigma} \varphi_{\nu}(x, y)
\end{aligned}
$$

$(\sigma$ is defined in $(3.54))$ and

$$
\begin{aligned}
& \left.\varphi_{\nu}(x, y+1)=(-1)^{\nu_{3}} \varphi_{\nu} x, y\right), \\
& \varphi_{\nu}(x, y+\tau)=(-1)^{\nu_{1}} e^{-\pi i n \tau-2 \pi i n y+\pi i n \eta-\pi i \sum_{k} \xi_{k}} \varphi_{\nu}(x, y) .
\end{aligned}
$$

Besides, it straightforwardly follows from the construction of the eigenvectors that

$$
\varphi_{\nu}(x+\eta, y)=e^{-\pi i \nu \eta} \varphi_{\nu}(x, y) .
$$

Below in section 4.2 we argue that $n+\nu_{3}=-\nu$ and

$$
2 \sigma=n+\nu_{1}+\nu \tau
$$


(the sum rule (4.24)). Using these relations and the definition of $\sigma$, we can represent equations (3.64), (3.65), (3.66) in the form

$$
\begin{aligned}
& \varphi_{\nu}(x+1, y)=(-1)^{\nu} \varphi_{\nu}(x, y), \\
& \varphi_{\nu}(x+\tau, y)=e^{-\pi i \nu \tau} \varphi_{\nu}(x, y), \\
& \varphi_{\nu}(x+\eta, y)=e^{-\pi i \nu \eta} \varphi_{\nu}(x, y)
\end{aligned}
$$

and

$$
\begin{aligned}
& \varphi_{\nu}(x, y+1)=(-1)^{n+\nu} \varphi_{\nu}(x, y), \\
& \varphi_{\nu}(x, y+\tau)=(-1)^{n} e^{-\pi i n \tau-2 \pi i n y+\pi i \nu \tau-2 \pi i \sum_{j} v_{j}} \varphi_{\nu}(x, y) .
\end{aligned}
$$

We know that $\varphi_{\nu}(x, y)$ is an entire function of $y$ and may have poles in $x$ at the points $x=-l \eta$ in the fundamental domain, where $l=0, \ldots Q / 2$ for even $Q$ and $l=0, \ldots, Q$ for odd $Q$. It then follows from (3.67), (3.68) and these properties that the poles in $x$ actually cancel and as a function of $y \varphi_{\nu}(x, y)$ is a theta function of order $n$, i.e. it has $n$ zeros $y_{j}$ in the fundamental domain:

$$
\varphi_{\nu}(x, y)=b_{\nu} e^{\pi i \nu(y-x)} \prod_{j=1}^{n} \theta_{1}\left(y-y_{j} \mid \tau\right)
$$

with the condition

$$
\sum_{j=1}^{n} y_{j}=-\sum_{j=1}^{n} v_{j}
$$

We conjecture that $y_{j}=-v_{j}$. This conjecture is supported by numerical calculations for $N=2,4$. Remarkably, the dependence on $x$ and $y$ factorizes.

\section{The $Q$-operator and the sum rule}

Here we construct the Baxter's $Q$-operator. This construction is necessary to obtain a sum rule, which is an additional requirement to the eigenvectors besides the Bethe equations. This section is not directly connected with what follows and is included for completeness.

\subsection{Construction of the $Q$-operator}

A minor modification of the formulas in sections 2.2 and 2.3 leads to the construction of the $Q$-operator. We begin with the construction of the right $Q$-operator $\mathrm{Q}_{R}(u)$. Basically, one should shift $s \rightarrow s-u, t \rightarrow t+u$ and consider the $L$-operators

$$
\mathrm{L}^{ \pm}(u)=M_{l}^{-1} \mathrm{~L}(u) M_{l \pm 1}=\left(\begin{array}{ll}
\mathrm{a}^{ \pm}(u) & \mathrm{b}^{ \pm}(u) \\
\mathrm{c}^{ \pm}(u) & \mathrm{d}^{ \pm}(u)
\end{array}\right),
$$

where

$$
M_{l}=\left(\begin{array}{cc}
\theta_{1}\left(s_{l} \mid 2 \tau\right) & \gamma_{l} \theta_{1}\left(t_{l} \mid 2 \tau\right) \\
\theta_{4}\left(s_{l} \mid 2 \tau\right) & \gamma_{l} \theta_{4}\left(t_{l} \mid 2 \tau\right)
\end{array}\right)
$$


The identities from section 2.2 allow one to prove the following important relations:

$$
\begin{aligned}
\mathrm{c}^{ \pm}(u-\xi)\left|\phi\left(s_{l} \pm(u-\xi)\right)\right\rangle & =0, \\
\mathrm{a}^{ \pm}(u-\xi)\left|\phi\left(s_{l} \pm(u-\xi)\right)\right\rangle & =\theta_{1}(u-\xi+\eta \mid \tau)\left|\phi\left(s_{l} \pm(u-\eta-\xi)\right)\right\rangle, \\
\mathrm{d}^{ \pm}(u-\xi)\left|\phi\left(s_{l} \pm(u-\xi)\right)\right\rangle & =\theta_{1}(u-\xi \mid \tau)\left|\phi\left(s_{l} \pm(u+\eta-\xi)\right)\right\rangle .
\end{aligned}
$$

Let $\epsilon_{i}= \pm 1, i=1, \ldots, N$ be such that $\sum_{i=1}^{N} \epsilon_{i}=0$ (for even $N$ this is always possible) and set $e_{m}=\sum_{i=1}^{m} \epsilon_{i}, e_{0}=e_{N}=0$. Let us introduce a family of vectors

$$
\left|\omega\left(u ; \epsilon_{1}, \ldots, \epsilon_{N}\right)\right\rangle=\bigotimes_{i=0, \ldots, N-1} \mid \phi\left(s_{l+e_{i}}-\epsilon_{i+1}\left(u-\xi_{i}\right)\right\rangle .
$$

Then the relations (4.3) imply that

$$
\mathrm{T}(u)\left|\omega\left(u ; \epsilon_{1}, \ldots, \epsilon_{N}\right)\right\rangle=a(u)\left|\omega\left(u-\eta ; \epsilon_{1}, \ldots, \epsilon_{N}\right)\right\rangle+d(u)\left|\omega\left(u+\eta ; \epsilon_{1}, \ldots, \epsilon_{N}\right)\right\rangle,
$$

where

$$
a(u)=\prod_{i=1}^{N} \theta_{1}\left(u-\xi_{i}+\eta\right), \quad d(u)=\prod_{i=1}^{N} \theta_{1}\left(u-\xi_{i}\right) .
$$

The vectors $\left|\omega\left(u ; \epsilon_{1}, \ldots, \epsilon_{N}\right)\right\rangle$ can be regarded as columns of an operator $\mathrm{Q}_{R}(u)$ (a "pre$Q$-operator) which, therefore, satisfies the relation

$$
\mathrm{T}(u) \mathrm{Q}_{R}(u)=a(u) \mathrm{Q}_{R}(u-\eta)+d(u) \mathrm{Q}_{R}(u+\eta) .
$$

Since $s$ can be any complex number and $\epsilon_{1}, \ldots, \epsilon_{N}$ is any set of numbers \pm 1 (such that their sum is zero), the set of all possible vectors $\left|\omega\left(u ; \epsilon_{1}, \ldots, \epsilon_{N}\right)\right\rangle$ spans the total $2^{N}$-dimensional quantum space $\mathcal{H}$ of the model.

The construction of the left $Q$-operator, $\mathrm{Q}_{L}(u)$, is similar. We define

$$
\overline{\mathrm{L}}^{ \pm}(u)=\bar{M}_{l}^{-1} \mathrm{~L}(u) \bar{M}_{l \pm 1}=\left(\begin{array}{ll}
\overline{\mathrm{a}}^{ \pm}(u) & \overline{\mathrm{b}}^{ \pm}(u) \\
\overline{\mathrm{c}}^{ \pm}(u) & \overline{\mathrm{d}}^{ \pm}(u)
\end{array}\right),
$$

where

$$
\bar{M}_{l}=\left(\begin{array}{cc}
\gamma_{l} \theta_{1}\left(s_{l} \mid 2 \tau\right) & \theta_{1}\left(t_{l} \mid 2 \tau\right) \\
\gamma_{l} \theta_{4}\left(s_{l} \mid 2 \tau\right) & \theta_{4}\left(t_{l} \mid 2 \tau\right)
\end{array}\right) .
$$

The identities from section 2.2 allow one to prove the relations

$$
\begin{aligned}
& \left\langle\phi^{\perp}\left(t_{l \pm 1} \pm(u-\xi)\right)\right| \overline{\mathrm{b}}^{ \pm}(u-\xi)=0, \\
& \left\langle\phi^{\perp}\left(t_{l \pm 1} \pm(u-\xi)\right)\right| \overline{\mathrm{a}}^{ \pm}(u-\xi)=\theta_{1}(u-\xi+\eta \mid \tau)\left\langle\phi^{\perp}\left(t_{l \pm 1} \pm(u-\eta-\xi)\right)\right|, \\
& \left\langle\phi^{\perp}\left(t_{l \pm 1} \pm(u-\xi)\right)\right| \overline{\mathrm{d}}^{ \pm}(u-\xi)=\theta_{1}(u-\xi \mid \tau)\left\langle\phi^{\perp}\left(t_{l \pm 1} \pm(u+\eta-\xi)\right)\right| .
\end{aligned}
$$

We introduce a family of dual vectors

$$
\left\langle\bar{\omega}\left(u ; \epsilon_{1}, \ldots, \epsilon_{N}\right)\right|={\underset{\bigotimes}{i=1, \ldots, N}}_{\overrightarrow{1}}\left\langle\phi ^ { \perp } \left( t_{l+e_{i}}+\epsilon_{i}\left(u-\xi_{i}\right) \mid,\right.\right.
$$


which satisfy

$$
\left\langle\bar{\omega}\left(u ; \epsilon_{1}, \ldots, \epsilon_{N}\right)\right| \mathrm{T}(u)=a(u)\left\langle\bar{\omega}\left(u-\eta ; \epsilon_{1}, \ldots, \epsilon_{N}\right)\right|+d(u)\left\langle\bar{\omega}\left(u+\eta ; \epsilon_{1}, \ldots, \epsilon_{N}\right)\right|,
$$

and regard them as rows of an operator $Q_{L}(u)$ which, therefore, satisfies the relation

$$
\mathrm{Q}_{L}(u) \mathrm{T}(u)=a(u) \mathrm{Q}_{L}(u-\eta)+d(u) \mathrm{Q}_{L}(u+\eta) .
$$

Now, using the argument of Baxter's works (see [4]), one can prove the commutation relation

$$
\mathrm{Q}_{L}(u) \mathrm{Q}_{R}(v)=\mathrm{Q}_{L}(v) \mathrm{Q}_{R}(u)
$$

and define the $Q$-operator $\mathrm{Q}(u)=\mathrm{Q}_{R}(u) \mathrm{Q}_{R}^{-1}\left(u_{0}\right)=\mathrm{Q}_{L}^{-1}\left(u_{0}\right) \mathrm{Q}_{L}(u)$, where $u_{0}$ is some point for which the left and right $Q$-operators are invertible. It then follows that $[\mathrm{Q}(u), \mathrm{Q}(v)]=$ $[\mathrm{Q}(u), \mathrm{T}(v)]=0$ and the $Q$-operator obeys the $T Q$-relation

$$
\mathrm{T}(u) \mathrm{Q}(u)=a(u) \mathrm{Q}(u-\eta)+d(u) \mathrm{Q}(u+\eta)
$$

which is the main property of the $Q$-operator.

\subsection{The sum rule}

It is straightforward to check that

$$
\begin{aligned}
& \mathrm{Q}_{R}(u+1)=\mathrm{U}_{3} \mathrm{Q}_{R}(u), \\
& \mathrm{Q}_{R}(u+\tau)=e^{-\pi i c(u) / 2} \mathrm{U}_{1} \mathrm{Q}_{R}(u), \\
& \mathrm{Q}_{L}(u+1)=\mathrm{Q}_{L}(u) \mathrm{U}_{3}, \\
& \mathrm{Q}_{L}(u+\tau)=e^{-\pi i c(u) / 2} \mathrm{Q}_{L}(u) \mathrm{U}_{1},
\end{aligned}
$$

where $\mathrm{U}_{a}$ are operators defined in (2.18). It follows from these relations that $\left[\mathrm{Q}(u), \mathrm{U}_{a}\right]=0$ and

$$
\begin{aligned}
& \mathrm{Q}(u+1)=\mathrm{U}_{3} \mathrm{Q}(u), \\
& \mathrm{Q}(u+\tau)=e^{-\pi i c(u) / 2} \mathrm{U}_{1} \mathrm{Q}(u) .
\end{aligned}
$$

Let $Q(u)$ be the eigenvalue of the operator $\mathrm{Q}(u)$ on a common eigenfunction with the operators $U_{a}$. As we have seen before, the eigenvalues of the operators $U_{a}$ are $(-1)^{\nu_{a}}$, where $\nu_{a}=0,1$. We can write

$$
\begin{aligned}
& Q(u+1)=(-1)^{\nu_{3}} Q(u), \\
& Q(u+\tau)=(-1)^{\nu_{1}} e^{-\pi i c(u) / 2} Q(u) .
\end{aligned}
$$

It follows from (4.18) that the entire function $Q(u)$ has exactly $n$ zeros $v_{i}$ in the fundamental domain. Let us consider a function

$$
F(u)=\frac{Q(u)}{\prod_{i=1}^{n} \theta_{1}\left(u-v_{i} \mid \tau\right)} .
$$

It is an entire function of $u$, and equations (4.18) imply that

$$
F(u+1)=(-1)^{n+\nu_{3}} F(u), \quad F(u+\tau)=(-1)^{n+\nu_{1}} e^{-2 \pi i \sigma} F(u),
$$


where $\sigma$ is defined in (3.54). It follows from these properties that $F(u)$ is the exponential function $F(u)=e^{\pi i\left(n+\nu_{3}\right) u}$ and

$$
2 \sigma=n+\nu_{1}-\left(n+\nu_{3}\right) \tau
$$

Therefore,

$$
Q(u)=e^{\pi i\left(n+\nu_{3}\right) u} \prod_{i=1}^{n} \theta_{1}\left(u-v_{i} \mid \tau\right) .
$$

It remains to identify zeros of the $Q(u)$ with Bethe roots. Writing the $T Q$ relation (4.14) for the eigenvalues,

$$
T\left(u ; v_{1}, \ldots, v_{n}\right)=a(u) \frac{Q(u-\eta)}{Q(u)}+d(u) \frac{Q(u+\eta)}{Q(u)},
$$

and using the fact that $T\left(u ; v_{1}, \ldots, v_{n}\right)$ does not have poles at $u=v_{i}$, one obtains the Bethe equations

$$
e^{-2 \pi i\left(n+\nu_{3}\right) \eta} \frac{a\left(v_{j}\right)}{d\left(v_{j}\right)}=\prod_{k=1, \neq j} \frac{\theta_{1}\left(v_{j}-v_{k}+\eta \mid \tau\right)}{\theta_{1}\left(v_{j}-v_{k}-\eta \mid \tau\right)} .
$$

Comparing with (3.25), one identifies

$$
\nu=-\left(n+\nu_{3}\right)
$$

In addition, we conclude that the Bethe roots have to satisfy the sum rule

$$
\sum_{i=1}^{n} v_{i}=\frac{1}{2} \sum_{k=1}^{N} \xi_{k}-\frac{1}{2} n \eta+\frac{1}{2}\left(n+\nu_{1}+\nu \tau\right),
$$

and $\nu$ can only take integer values. Taking this into account, one may say that the eigenvector of the transfer matrix is determined by a solution of the extended system of Bethe equations, i.e., the system (3.25) supplemented by the sum rule (4.24) for the $n+1$ unknown variables $\left\{\nu, v_{1}, \ldots, v_{n}\right\}$.

It should be noted that the $Q$-operator has some eigenvectors which are not eigenvectors of the spin reflection operator (see $[14,16]$ ). For such states, the sum rule is not valid.

\section{Scalar products of Bethe vectors}

In this section, we obtain a system of linear equations for scalar products of the on-shell and off-shell Bethe vectors. Basically, we follow the method of [49]. However, for models with the 8 -vertex $R$-matrix, some generalization is required.

\subsection{The notation}

In this section we denote $\bar{u}=\left\{u_{1}, \ldots, u_{n+1}\right\}, \bar{v}=\left\{v_{1}, \ldots, v_{n}\right\}, \bar{w}=\left\{w_{1}, \ldots, w_{n+1}\right\}$. We also denote $\bar{u}_{j}=\bar{u} \backslash u_{j}, \bar{v}_{k}=\bar{v} \backslash v_{k}$ and so on. Let us also introduce the functions

$$
g(u, v)=\frac{\theta_{1}(\eta)}{\theta_{1}(u-v)}, \quad f(u, v)=\frac{\theta_{1}(u-v+\eta)}{\theta_{1}(u-v)}, \quad h(u, v)=\frac{\theta_{1}(u-v+\eta)}{\theta_{1}(\eta)} .
$$


Here and below in this section $\theta_{1}(x) \equiv \theta_{1}(x \mid \tau)$. Observe that

$$
g(u, v)=-g(v, u), \quad f(u, v)=g(u, v) h(u, v), \quad h(u, u)=1 .
$$

In order to make the formulas more compact, we use a shorthand notation for products of these functions. Namely, if any of them depends on a (sub)set of variables, then one should take a product with respect to the corresponding (sub)set. For example,

$$
f(u, \bar{v})=\prod_{j=1}^{n} f\left(u, v_{j}\right), \quad h\left(\bar{w}, u_{k}\right)=\prod_{j=1}^{n+1} h\left(w_{j}, u_{k}\right), \quad g\left(u_{k}, \bar{u}_{k}\right)=\prod_{j=1, \neq k}^{n+1} g\left(u_{k}, u_{j}\right)
$$

and so on. We stress that this convention is applied to the functions (5.1) only.

Finally, the functions $a(u), d(u)$ are defined in (4.5).

\subsection{A system of linear equations for scalar products}

We now proceed directly to the derivation of the system of equations for scalar products. Using definitions (5.1) and convention (5.3) we rewrite equation (3.22) for the action of the operator $\mathrm{T}(u)=A_{l, l}(u)+D_{l, l}(u)$ as follows:

$$
\begin{aligned}
\mathrm{T}\left(u_{j}\right)\left|\Psi^{l}\left(\bar{u}_{j}\right)\right\rangle=\sum_{k=1}^{n+1}[ & a\left(u_{k}\right) f\left(\bar{u}_{k}, u_{k}\right) \frac{\theta_{1}\left(u_{j}-u_{k}+\tau_{l+1}+\frac{1}{2}\right)}{h\left(u_{j}, u_{k}\right) \theta_{1}\left(\tau_{l+1}+\frac{1}{2}\right)}\left|\Psi^{l+1}\left(\bar{u}_{k}\right)\right\rangle \\
& \left.+d\left(u_{k}\right) f\left(u_{k}, \bar{u}_{k}\right) \frac{\theta_{1}\left(u_{j}-u_{k}+\tau_{l-1}+\frac{1}{2}\right)}{h\left(u_{k}, u_{j}\right) \theta_{1}\left(\tau_{l-1}+\frac{1}{2}\right)}\left|\Psi^{l-1}\left(\bar{u}_{k}\right)\right\rangle\right] .
\end{aligned}
$$

Set

$$
X_{j}^{l}=\left\langle\Psi_{\nu}(\bar{v}) \mid \Psi^{l}\left(\bar{u}_{j}\right)\right\rangle,
$$

where $\left\langle\Psi_{\nu}(\bar{v})\right|$ is a dual on-shell Bethe vector:

$$
\left\langle\Psi_{\nu}(\bar{v})\right| \mathrm{T}\left(u_{j}\right)=T_{\nu}\left(u_{j}, \bar{v}\right)\left\langle\Psi_{\nu}(\bar{v})\right| \quad \text { for all } u_{j} \in \mathbb{C} .
$$

This means that the parameters $\bar{v}$ are supposed to satisfy the Bethe equations (3.25). The eigenvalue is given by

$$
T_{\nu}\left(u_{j}, \bar{v}\right)=e^{i \pi \eta \nu} a\left(u_{j}\right) f\left(\bar{v}, u_{j}\right)+e^{-i \pi \eta \nu} d\left(u_{j}\right) f\left(u_{j}, \bar{v}\right) .
$$

Multiplying (5.4) from the left by $\left\langle\Psi_{\nu}(\bar{v})\right|$ and using (5.6), we obtain:

$$
\begin{aligned}
& \sum_{k=1}^{n+1}\left[a\left(u_{k}\right) f\left(\bar{u}_{k}, u_{k}\right) \frac{\theta_{1}\left(u_{j}-u_{k}+\tau_{l+1}+\frac{1}{2}\right)}{h\left(u_{j}, u_{k}\right) \theta_{1}\left(\tau_{l+1}+\frac{1}{2}\right)} X_{k}^{l+1}\right. \\
& \left.\quad+d\left(u_{k}\right) f\left(u_{k}, \bar{u}_{k}\right) \frac{\theta_{1}\left(u_{j}-u_{k}+\tau_{l-1}+\frac{1}{2}\right)}{h\left(u_{k}, u_{j}\right) \theta_{1}\left(\tau_{l-1}+\frac{1}{2}\right)} X_{k}^{l-1}-\delta_{j k} T_{\nu}\left(u_{j}, \bar{v}\right) X_{k}^{l}\right]=0 .
\end{aligned}
$$

We recall that $\tau_{l}=\frac{1}{2}\left(s_{l}+t_{l}\right)=x-\frac{1}{2}+l \eta$. This is a homogeneous system of linear equations for the scalar products, similar to the one familiar from the rational and trigonometric cases [49] but with an additional integer parameter $l$. 
In what follows we assume that $\eta$ is a rational number $\eta=2 P / Q(3.59)$. In this case $X_{k}^{l}$ and coefficients of the system (5.8) are $Q$-periodic in $l$ and the index $l$ in (5.8) should be understood modulo $Q(l+Q=l)$. Therefore, (5.8) is a homogeneous system of $(n+1) Q$ linear equations for $(n+1) Q$ unknown variables $X_{k}^{l}, k=1, \ldots n+1, l \in \mathbb{Z}_{Q}, \mathbb{Z}_{Q}=$ $\{0, \ldots, Q-1\}$. In the next subsection we show that this system has non-trivial solutions.

Below we consider only the case when the number of Bethe parameters in the two vectors in the scalar product (5.5) is the same and is equal to $N / 2$ although for rational $\eta$ this number can also take other values (see (3.61)).

\subsection{Transformation of the system and solvability}

Since the obtained system of equations (5.8) is homogeneous, its solutions (if any) are ambiguous. Namely, if $X_{k}^{l}$ is a solution to the system, then $\phi(\bar{v}, \bar{u}) X_{k}^{l}$ is also a solution to the system, where $\phi(\bar{v}, \bar{u})$ is an arbitrary function of the variables $\bar{v}$ and $\bar{u}$. In order to minimize possible arbitrariness, we, following the method of [49], transform the system to a new (equivalent) form and show that the solutions of the new system are determined up to a function that depends on the variables $\bar{v}$, but does not depend on the parameters $\bar{u}$. As a byproduct, we prove that the rank of the system is less than $(n+1) Q$, and therefore, it does have nontrivial solutions.

Let us introduce $(n+1) \times(n+1)$ matrices $W^{l}$ with the entries

$$
W_{j k}^{l}=g\left(u_{k}, w_{j}\right) \frac{g\left(u_{k}, \bar{u}_{k}\right)}{g\left(u_{k}, \bar{w}\right)} \theta_{1}\left(u_{k}-w_{j}-S-\tau_{l}-\frac{1}{2}\right), \quad j, k=1, \ldots, n+1, \quad l \in \mathbb{Z}_{Q},
$$

where $\bar{w}=\left\{w_{1}, \ldots, w_{n+1}\right\}$ are arbitrary pairwise distinct complex numbers and

$$
S=\sum_{j=1}^{n+1}\left(u_{j}-w_{j}\right)
$$

The matrix $W^{l}$ is nothing else than an elliptic Cauchy matrix multiplied by a diagonal matrix from the right. The determinant of the elliptic Cauchy matrix is given by

$$
\begin{aligned}
& \operatorname{det}_{1 \leq i, j \leq n+1} \Phi\left(u_{i}-w_{j}, \lambda\right)=\frac{\left(\theta_{1}^{\prime}(0)\right)^{n+1}}{\theta_{1}\left(\lambda+\sum_{i=1}^{n+1}\left(u_{i}-w_{j}\right)\right)} \theta_{1}(\lambda) \\
& \times \frac{\prod_{p<q} \theta_{1}\left(u_{p}-u_{q}\right) \theta_{1}\left(w_{q}-w_{p}\right)}{\prod_{r, s} \theta_{1}\left(u_{r}-w_{s}\right)},
\end{aligned}
$$

where $\Phi$ is the function (3.20). It is seen from this formula that $\operatorname{det} W^{l} \neq 0$ if all $u_{j}$ and $w_{j}$ are distinct and $\tau_{l}+\frac{1}{2} \neq 0, S+\tau_{l}+\frac{1}{2} \neq 0$ modulo the lattice spanned by 1 and $\tau$.

Multiplying the system (5.8) from the left by $W^{l}$ we obtain:

$$
\sum_{k=1}^{n+1}\left[\frac{a\left(u_{k}\right) f\left(\bar{u}_{k}, u_{k}\right)}{\theta_{1}\left(\tau_{l+1}+\frac{1}{2}\right)} E_{j k}^{+} X_{k}^{l+1}+\frac{d\left(u_{k}\right) f\left(u_{k}, \bar{u}_{k}\right)}{\theta_{1}\left(\tau_{l-1}+\frac{1}{2}\right)} E_{j k}^{-} X_{k}^{l-1}-W_{j k}^{l} T_{\nu}\left(u_{k}, \bar{v}\right) X_{k}^{l}\right]=0 .
$$

Here

$$
E_{j k}^{ \pm}=\theta_{1}( \pm \eta) \sum_{m=1}^{n+1} W_{j m}^{l} \frac{\theta_{1}\left(u_{m}-u_{k}+\tau_{l \pm 1}+\frac{1}{2}\right)}{\theta_{1}\left(u_{m}-u_{k} \pm \eta\right)}
$$


As soon as the matrix $W^{l}$ is non-degenerate, the new system is equivalent to the previous one.

The sum (5.13) can be calculated via an auxiliary contour integral. Let

$$
I^{ \pm}=\frac{\theta_{1}( \pm \eta) \theta_{1}^{\prime}(0)}{2 \pi i} \times \oint \frac{\theta_{1}\left(z-u_{k}+\tau_{l} \pm \eta+\frac{1}{2}\right)}{\theta_{1}\left(z-u_{k} \pm \eta\right)} \frac{\theta_{1}\left(z-w_{j}-S-\tau_{l}-\frac{1}{2}\right)}{\theta_{1}\left(z-w_{j}\right)} \prod_{p=1}^{n+1} \frac{\theta_{1}\left(z-w_{p}\right)}{\theta_{1}\left(z-u_{p}\right)} d z
$$

where the integration goes along the boundary of the fundamental parallelogram. Then $I^{ \pm}=0$ due to the periodicity of the integrand. On the other hand, this integral can be calculated as sum of the residues in the interior of the contour. It is easy to see that the sum of the residues at the points $z=u_{m}$ gives exactly $E_{j k}^{ \pm}$. One more contribution comes from the pole at $z-u_{k} \pm \eta=0$. Thus we arrive at

$$
0=E_{j k}^{ \pm}+\theta_{1}( \pm \eta) \theta_{1}\left(\tau_{l}+\frac{1}{2}\right) \frac{\theta_{1}\left(u_{k}-w_{j}-S-\tau_{l \pm 1}-\frac{1}{2}\right)}{\theta_{1}\left(u_{k}-w_{j} \mp \eta\right)} \prod_{p=1}^{n+1} \frac{\theta_{1}\left(u_{k}-w_{p} \mp \eta\right)}{\theta_{1}\left(u_{k}-u_{p} \mp \eta\right)},
$$

leading to

$$
\begin{aligned}
& E_{j k}^{+}=\theta_{1}\left(u_{k}-w_{j}-S-\tau_{l+1}-\frac{1}{2}\right) \frac{\theta_{1}\left(\tau_{l}+\frac{1}{2}\right)}{h\left(w_{j}, u_{k}\right)} \frac{h\left(\bar{w}, u_{k}\right)}{h\left(\bar{u}, u_{k}\right)}, \\
& E_{j k}^{-}=\theta_{1}\left(u_{k}-w_{j}-S-\tau_{l-1}-\frac{1}{2}\right) \frac{\theta_{1}\left(\tau_{l}+\frac{1}{2}\right)}{h\left(u_{k}, w_{j}\right)} \frac{h\left(u_{k}, \bar{w}\right)}{h\left(u_{k}, \bar{u}\right)} .
\end{aligned}
$$

Substituting these expressions into (5.12), we obtain:

$$
\begin{aligned}
& \sum_{k=1}^{n+1} g\left(u_{k}, \bar{u}_{k}\right)\left[(-1)^{n} a\left(u_{k}\right) h\left(\bar{w}, u_{k}\right) \frac{\theta_{1}\left(u_{k}-w_{j}-S_{l+1}\right)}{\theta_{1}\left(\tau_{l+1}+\frac{1}{2}\right) h\left(w_{j}, u_{k}\right)} X_{k}^{l+1}\right. \\
& \quad+d\left(u_{k}\right) h\left(u_{k}, \bar{w}\right) \frac{\theta_{1}\left(u_{k}-w_{j}-S_{l-1}\right)}{\theta_{1}\left(\tau_{l-1}+\frac{1}{2}\right) h\left(u_{k}, w_{j}\right)} X_{k}^{l-1} \\
& \left.\quad-\frac{\theta_{1}\left(u_{k}-w_{j}-S_{l}\right)}{\theta_{1}\left(\tau_{l}+\frac{1}{2}\right)} \frac{g\left(u_{k}, w_{j}\right)}{g\left(u_{k}, \bar{w}\right)}\left(e^{i \pi \eta \nu} a\left(u_{k}\right) f\left(\bar{v}, u_{k}\right)+e^{-i \pi \eta \nu} d\left(u_{k}\right) f\left(u_{k}, \bar{v}\right)\right) X_{k}^{l}\right]=0
\end{aligned}
$$

where

$$
S_{l}=S+\tau_{l}+\frac{1}{2} .
$$

This is a new system of equations which contains the set of arbitrary complex parameters $\bar{w}$. Note that they may depend on $l$.

Let us set $\bar{w}_{n+1}=\bar{v}$, while the parameter $w_{n+1}$ remains free. Set

$$
P_{l}=\sum_{j=1}^{n+1} u_{j}-\sum_{j=1}^{n} v_{j}+\tau_{l}+\frac{1}{2}
$$

Consider equations (5.16) for $j=n+1$. We have $Q$ equations of the form

$$
\begin{aligned}
& \sum_{k=1}^{n+1}\left[(-1)^{n} a\left(u_{k}\right) h\left(\bar{v}, u_{k}\right)\left(\frac{\theta_{1}\left(u_{k}-P_{l+1}\right)}{\theta_{1}\left(\tau_{l+1}+\frac{1}{2}\right)} X_{k}^{l+1}-e^{i \pi \eta \nu} \frac{\theta_{1}\left(u_{k}-P_{l}\right)}{\theta_{1}\left(\tau_{l}+\frac{1}{2}\right)} X_{k}^{l}\right)\right. \\
& \left.\quad+d\left(u_{k}\right) h\left(u_{k}, \bar{v}\right)\left(\frac{\theta_{1}\left(u_{k}-P_{l-1}\right)}{\theta_{1}\left(\tau_{l-1}+\frac{1}{2}\right)} X_{k}^{l-1}-e^{-i \pi \eta \nu} \frac{\theta_{1}\left(u_{k}-P_{l}\right)}{\theta_{1}\left(\tau_{l}+\frac{1}{2}\right)} X_{k}^{l}\right)\right] g\left(u_{k}, \bar{u}_{k}\right)=0 .
\end{aligned}
$$


It is easy to see that these equations are linearly dependent. Indeed, if we multiply (5.19) by $e^{-i l \pi \eta \mu}$, where $\mu \in \mathbb{Z}_{Q}$, and sum over $l \in \mathbb{Z}_{Q}$, we immediately see that the l.h.s. vanishes for $\mu=\nu$ (and does not vanish for $\mu \neq \nu$ ). Hence, the system does have non-trivial solutions.

Consider the remaining system for $j<n+1$ and $\bar{w}_{n+1}=\bar{v}$. The parameter $w_{n+1}$ is still free and we denote it by $w$. Then we have $n Q$ equations of the form

$$
\begin{aligned}
\sum_{k=1}^{n+1} g\left(u_{k}, \bar{u}_{k}\right)[ & \mathcal{A}_{k}\left(\frac{h\left(w, u_{k}\right)}{h\left(v_{j}, u_{k}\right)} \frac{\theta_{1}\left(u_{k}-v_{j}+w-P_{l+1}\right)}{\theta_{1}\left(\tau_{l+1}+\frac{1}{2}\right)} X_{k}^{l+1}\right. \\
& \left.-e^{i \pi \eta \nu} \frac{g\left(v_{j}, u_{k}\right)}{g\left(w, u_{k}\right)} \frac{\theta_{1}\left(u_{k}-v_{j}+w-P_{l}\right)}{\theta_{1}\left(\tau_{l}+\frac{1}{2}\right)} X_{k}^{l}\right) \\
& +\mathcal{D}_{k}\left(\frac{h\left(u_{k}, w\right)}{h\left(u_{k}, v_{j}\right)} \frac{\theta_{1}\left(u_{k}-v_{j}+w-P_{l-1}\right)}{\theta_{1}\left(\tau_{l-1}+\frac{1}{2}\right)} X_{k}^{l-1}\right. \\
& \left.\left.-e^{-i \pi \eta \nu} \frac{g\left(u_{k}, v_{j}\right)}{g\left(u_{k}, w\right)} \frac{\theta_{1}\left(u_{k}-v_{j}+w-P_{l}\right)}{\theta_{1}\left(\tau_{l}+\frac{1}{2}\right)} X_{k}^{l}\right)\right]=0
\end{aligned}
$$

where $j=1, \ldots, n, l=0,1, \ldots, Q-1$ and

$$
\mathcal{A}_{k}=(-1)^{n} a\left(u_{k}\right) h\left(\bar{v}, u_{k}\right), \quad \mathcal{D}_{k}=d\left(u_{k}\right) h\left(u_{k}, \bar{v}\right) .
$$

Now let us transform the system (5.20) further. We will take advantage of the fact that the parameter $w$ may depend on $l$ and take it to be

$$
w=l \eta+w_{0}+\check{U}, \quad \check{U}=\sum_{j=1}^{n+1} u_{j},
$$

where $w_{0}$ is an $l$-independent free parameter. Then the system (5.20) becomes:

$$
\begin{aligned}
\sum_{k=1}^{n+1} g\left(u_{k}, \bar{u}_{k}\right)[ & \mathcal{A}_{k}\left(\frac{\theta_{1}\left(u_{k}-\check{U}-w_{0}-(l+1) \eta\right)}{\theta_{1}\left(\tau_{l+1}+\frac{1}{2}\right)} \frac{\theta_{1}\left(u_{k}-v_{j}+r-\eta\right)}{\theta_{1}\left(u_{k}-v_{j}-\eta\right) X_{k}^{l+1}}\right. \\
& \left.-e^{i \pi \eta \nu} \frac{\theta_{1}\left(u_{k}-\check{U}-w_{0}-l \eta\right)}{\theta_{1}\left(\tau_{l}+\frac{1}{2}\right)} \frac{\theta_{1}\left(u_{k}-v_{j}+r\right)}{\theta_{1}\left(u_{k}-v_{j}\right) X_{k}^{l}}\right) \\
& +\mathcal{D}_{k}\left(\frac{\theta_{1}\left(u_{k}-\check{U}-w_{0}-(l-1) \eta\right)}{\theta_{1}\left(\tau_{l-1}+\frac{1}{2}\right)} \frac{\theta_{1}\left(u_{k}-v_{j}+r+\eta\right)}{\theta_{1}\left(u_{k}-v_{j}+\eta\right) X_{k}^{l-1}}\right. \\
& \left.\left.-e^{-i \pi \eta \nu} \frac{\theta_{1}\left(u_{k}-\check{U}-w_{0}-l \eta\right)}{\theta_{1}\left(\tau_{l}+\frac{1}{2}\right)} \frac{\theta_{1}\left(u_{k}-v_{j}+r\right)}{\theta_{1}\left(u_{k}-v_{j}\right) X_{k}^{l}}\right)\right]=0,
\end{aligned}
$$

where

$$
r=\sum_{p=1}^{n} v_{p}-\frac{1}{2}(s+t+1)+w_{0} .
$$


Multiplying these equations by $e^{-i l \pi \eta \mu}$ and summing over $l \in \mathbb{Z}_{Q}$, we obtain the following system of $n Q$ equations

$$
\begin{aligned}
\sum_{k=1}^{n+1} g\left(u_{k}, \bar{u}_{k}\right) & {\left[\mathcal{A}_{k}\left(e^{i \pi \eta \mu} \frac{\theta_{1}\left(u_{k}-v_{j}-\eta+r\right)}{\theta_{1}\left(u_{k}-v_{j}-\eta\right)}-e^{i \pi \eta \nu} \frac{\theta_{1}\left(u_{k}-v_{j}+r\right)}{\theta_{1}\left(u_{k}-v_{j}\right)}\right)\right.} \\
+ & \left.\mathcal{D}_{k}\left(e^{-i \pi \eta \mu} \frac{\theta_{1}\left(u_{k}-v_{j}+\eta+r\right)}{\theta_{1}\left(u_{k}-v_{j}+\eta\right)}-e^{-i \pi \eta \nu} \frac{\theta_{1}\left(u_{k}-v_{j}+r\right)}{\theta_{1}\left(u_{k}-v_{j}\right)}\right)\right] Y_{k}^{(\mu)}=0
\end{aligned}
$$

for $(n+1) Q$ variables

$$
\begin{aligned}
Y_{k}^{(\mu)} & =\sum_{l \in \mathbb{Z}_{Q}} \frac{\theta_{1}\left(l \eta+w_{0}+\check{U}-u_{k}\right)}{\theta_{1}\left(\tau_{l}+\frac{1}{2}\right)} e^{-i l \pi \eta \mu} X_{k}^{l} \\
& =\sum_{l \in \mathbb{Z}_{Q}} \frac{\theta_{1}\left(l \eta+r+x+U_{k}-V\right)}{\theta_{1}(l \eta+x)} e^{-i l \pi \eta \mu} X_{k}^{l} .
\end{aligned}
$$

Here $\mu \in \mathbb{Z}_{Q}, x=\frac{1}{2}(s+t+1)$ and

$$
U_{k}=\sum_{a=1, \neq k} u_{a}=\check{U}-u_{k}, \quad V=\sum_{a=1}^{n} v_{a} .
$$

Comparing with (5.23), the system (5.25) is block-diagonal; the $Q$ diagonal blocks are numbered by $\mu \in \mathbb{Z}_{Q}$ and each block is a system of $n$ equations for $n+1$ variables $Y_{k}^{(\mu)}$. Note that $Y_{k}^{(\mu)}$ does not depend on $u_{k}$ but does depend on $r$ (although $X_{k}^{l}$ does not depend on it), so we can denote it as $Y_{k}^{(\mu)}=Y_{k}^{(\mu)}(r)$. We can represent the system (5.25) in the form

$$
\sum_{k=1}^{n+1} T_{i k}^{(\nu \mu)}(r) G_{k} Y_{k}^{(\mu)}(r)=0, \quad i=1, \ldots, n,
$$

where

$$
G_{k}=\frac{g\left(u_{k}, \bar{u}_{k}\right)}{g\left(u_{k}, \bar{v}\right)}
$$

and the matrix $T_{i k}^{(\nu \mu)}(r)$ is given by

$$
T_{i k}^{(\nu \mu)}(r)=\Phi\left(u_{k}-v_{i}, r\right)\left(T_{\nu}\left(u_{k}, \bar{v}\right)-T_{\mu}\left(u_{k}, \bar{v}_{i} \cup\left(v_{i}-r\right)\right)\right) .
$$

Here we have used the function $\Phi$ which is defined in (3.20). Another convenient representation of the matrix $T_{i k}^{(\nu \mu)}(r)$ is

$$
\begin{aligned}
T_{i k}^{(\nu \mu)}(r)= & \frac{\theta_{1}^{\prime}(0)}{\theta_{1}(r)}\left[a\left(u_{k}\right) f\left(\bar{v}, u_{k}\right)\left(e^{i \pi \eta \nu} \frac{\theta_{1}\left(u_{k}-v_{i}+r\right)}{\theta_{1}\left(u_{k}-v_{i}\right)}-e^{i \pi \eta \mu} \frac{\theta_{1}\left(u_{k}-v_{i}-\eta+r\right)}{\theta_{1}\left(u_{k}-v_{i}-\eta\right)}\right)\right. \\
& \left.+d\left(u_{k}\right) f\left(u_{k}, \bar{v}\right)\left(e^{-i \pi \eta \nu} \frac{\theta_{1}\left(u_{k}-v_{i}+r\right)}{\theta_{1}\left(u_{k}-v_{i}\right)}-e^{-i \pi \eta \mu} \frac{\theta_{1}\left(u_{k}-v_{i}+\eta+r\right)}{\theta_{1}\left(u_{k}-v_{i}+\eta\right)}\right)\right] .
\end{aligned}
$$


Note that

$$
T_{i k}^{(\nu \nu)}(0)=\frac{\partial T_{\nu}\left(u_{k}, \bar{v}\right)}{\partial v_{i}} .
$$

It is interesting to note that this form of the matrix is the same as in models with the 6 -vertex $R$-matrix (see $[36,43]$ ).

Let us show that the remaining equations (5.19) of the original system follow from (5.28). Indeed, making the Fourier transform of the system (5.19), we get $Q$ equations

$$
\sum_{k=1}^{n+1}\left(T_{\nu}\left(u_{k}\right)-T_{\mu}\left(u_{k}\right)\right) G_{k} Y_{k}^{(\mu)}(0)=0, \quad \mu \in \mathbb{Z}_{Q}
$$

(one of them, at $\mu=\nu$, is trivial). The system (5.28) should be satisfied for any $r$, including $r=0$ (at this point the system degenerates but the limit of the solution as $r \rightarrow 0$ is still a solution). At the same time

$$
\lim _{r \rightarrow 0}\left(\theta_{1}(r) T_{i k}^{(\nu \mu)}(r)\right)=T_{\nu}\left(u_{k}\right)-T_{\mu}\left(u_{k}\right),
$$

hence we see that equations (5.32) are satisfied for solutions of the system (5.28).

\subsection{Solution of the system}

Fixing some $k \in\{1, \ldots, n+1\}$ and writing the system (5.28) as

$$
\sum_{j=1, \neq k}^{n+1} T_{i j}^{(\nu \mu)}(r) G_{j} Y_{j}^{(\mu)}(r)=-T_{i k}^{(\nu \mu)}(r) G_{k} Y_{k}^{(\mu)}(r),
$$

we can use the Cramer's rule to write down the solution in the form

$$
G_{m} Y_{m}^{(\mu)}=(-1)^{k-m} G_{k} Y_{k}^{(\mu)} \frac{\operatorname{det}_{j \neq m} T_{i j}^{(\nu \mu)}(r)}{\operatorname{det}_{j \neq k} T_{i j}^{(\nu \mu)}(r)} .
$$

It is not difficult to verify that

$$
\frac{G_{k}}{G_{m}}=(-1)^{k-m} \frac{W_{n}\left(\bar{u}_{k}, \bar{v}\right)}{W_{n}\left(\bar{u}_{m}, \bar{v}\right)},
$$

where

$$
W_{n}\left(\bar{u}_{k}, \bar{v}\right)=\frac{\prod_{a<b, \neq k} \theta_{1}\left(u_{a}-u_{b}\right) \prod_{a^{\prime}>b^{\prime}} \theta_{1}\left(v_{a^{\prime}}-v_{b^{\prime}}\right)}{\prod_{p=1, \neq k}^{n+1} \prod_{p^{\prime}=1}^{n} \theta_{1}\left(u_{p}-v_{p^{\prime}}\right)} .
$$

It then follows from (5.33) that

$$
Y_{k}^{(\mu)} \frac{W_{n}\left(\bar{u}_{k}, \bar{v}\right)}{\operatorname{det}_{j \neq k} T_{i j}^{(\nu \mu)}(r)}=Y_{m}^{(\mu)} \frac{W_{n}\left(\bar{u}_{m}, \bar{v}\right)}{\operatorname{det}_{j \neq m} T_{i j}^{(\nu \mu)}(r)}
$$

for any $k, m=1, \ldots, n+1$. The left hand side does not depend on $u_{k}$ (but may depend on the other variables) while the right hand side does not depend on $u_{m}$ (but may depend on 
the other variables). Since this is true for any $k, m$, the both sides in fact do not depend on the variables $\bar{u}$ at all and we conclude that

$$
Y_{k}^{(\mu)}(r)=\phi^{(\nu, \mu)}(\bar{v}, r) \frac{\operatorname{det}_{j \neq k} T_{i j}^{(\nu \mu)}(r)}{W_{n}\left(\bar{u}_{k}, \bar{v}\right)},
$$

where $\phi^{(\nu, \mu)}(\bar{v}, r)$ is some symmetric function of the variables $\bar{v}$ and a function of $r, \mu, \nu$ (and of $x, y$ ). This is the solution of the system (5.25). Since $Y_{k}^{(\mu)}(r)$ depends also on $\nu$, below we will sometimes denote it as $Y_{k}^{(\nu, \mu)}(r)$.

\subsection{Trying to fix the ambiguity}

In the models with 6 -vertex $R$-matrix, the way to fix the unknown function $\phi$ in (5.36) is to compare the result (5.36) with a very particular case of the scalar product when $u_{k}=\xi_{k}$, $k=1, \ldots, n$ (and so $d\left(u_{k}\right)=0$ for all $k$ ). In this case the scalar product is known independently and is expressed through the partition function of the 6-vertex model with domain wall boundary conditions. The latter is known to have a determinant representation [64] which is to be compared with (5.36) in this particular case [49]. Unfortunately, this method does not work for the 8-vertex model, because the scalar products even in the particular case $u_{k}=\xi_{k}$ are not available in the explicit form.

In order to fix the function $\phi^{(\nu, \mu)}(\bar{v}, r)$, we are going to analyze transformation properties of both sides of equation (5.36) under shifts of the variables. This will allow us to fix the function $\phi^{(\nu, \mu)}(\bar{v}, r)$ only partially but, as we will see later, this is enough for specially normalized scalar products.

First let us analyze transformation properties under the shifts $r \rightarrow r+1, r \rightarrow r+\tau$. In this section $r$ is regarded as an independent free parameter. Clearly, $X_{k}^{l}$ does not depend on $r$. Therefore, from (5.26) we conclude that

$$
\begin{aligned}
& Y_{k}^{(\nu, \mu)}(r+1)=-Y_{k}^{(\nu, \mu)}(r), \\
& Y_{k}^{(\nu, \mu)}(r+\tau)=-e^{-\pi i \tau-2 \pi i\left(r+x+U_{k}-V\right)} Y_{k}^{(\nu, \mu+2)}(r) .
\end{aligned}
$$

It is straightforward to check that

$$
\begin{aligned}
& \operatorname{det}_{j \neq k} T_{i j}^{(\nu, \mu)}(r+1)=\operatorname{det}_{j \neq k} T_{i j}^{(\nu, \mu)}(r), \\
& \operatorname{det}_{j \neq k} T_{i j}^{(\nu, \mu)}(r+\tau)=e^{2 \pi i\left(V-U_{k}\right)} \operatorname{det}_{j \neq k} T_{i j}^{(\nu, \mu+2)}(r) .
\end{aligned}
$$

Substituting (5.37), (5.38) into the solution (5.36), one obtains:

$$
\begin{aligned}
\phi^{(\nu, \mu)}(\bar{v}, r+1) & =-\phi^{(\nu, \mu)}(\bar{v}, r), \\
\phi^{(\nu, \mu-2)}(\bar{v}, r+\tau) & =-e^{-\pi i \tau-2 \pi i r-2 \pi i x} \phi^{(\nu, \mu)}(\bar{v}, r) .
\end{aligned}
$$

Some more information about the function $\phi^{(\nu, \mu)}(\bar{v}, r)$ can be obtained by analyzing properties of the solution (5.36) under shifts of the parameters $s, t$. First let us consider the shifts $s \rightarrow s+1, t \rightarrow t+1$ and $s \rightarrow s+\tau, t \rightarrow t+\tau$ (i.e., $x \rightarrow x+1$ and $x \rightarrow x+\tau$ ). 
For this, we use the results of section 3.4. Note that since $r=V+w_{0}-x$, the shift of $x$ at constant $w_{0}$ should be accompanied by the corresponding shift of $r$. We have:

$$
\begin{aligned}
& X_{k}^{l}(x+1)=X_{k}^{l}(x), \\
& X_{k}^{l}(x+\tau)=e^{2 \pi i\left(U_{k}-V\right)} X_{k}^{l}(x)
\end{aligned}
$$

and thus

$$
\begin{aligned}
& Y_{k}^{(\mu)}(r-1, x+1)=-Y_{k}^{(\mu)}(r, x), \\
& Y_{k}^{(\mu)}(r-\tau, x+\tau)=-e^{\pi i \tau+2 \pi i x+2 \pi i\left(U_{k}-V\right)} Y_{k}^{(\mu-2)}(r, x) .
\end{aligned}
$$

We also have

$$
\operatorname{det}_{j \neq k} T_{i j}^{(\nu \mu)}(r-\tau)=e^{2 \pi i\left(U_{k}-V\right)} \operatorname{det}_{j \neq k} T_{i j}^{(\nu, \mu-2)}(r) .
$$

Substituting this into the solution (5.36), we find:

$$
\begin{aligned}
\phi^{(\nu, \mu)}(r-1, x+1) & =-\phi^{(\nu, \mu)}(r, x), \\
\phi^{(\nu, \mu+2)}(r-\tau, x+\tau) & =-e^{\pi i \tau+2 \pi i x} \phi^{(\nu, \mu)}(r, x) .
\end{aligned}
$$

For brevity, the dependence on $\bar{v}$ is omitted in the notation. The other possibility is to shift $w_{0}$ simultaneously with $x$, so that $r$ remains constant. In this way we get:

$$
\begin{aligned}
& Y_{k}^{(\mu)}(r, x+1)=Y_{k}^{(\mu)}(r, x), \\
& Y_{k}^{(\mu)}(r, x+\tau)=e^{-2 \pi i r} Y_{k}^{(\mu)}(r, x), \\
& Y_{k}^{(\mu)}(r, x+\eta)=e^{i \pi \eta(\mu-\nu)} Y_{k}^{(\mu)}(r, x) .
\end{aligned}
$$

These properties imply the following properties of the function $\phi^{(\nu, \mu)}(r, x)$ under shifts of $x$ :

$$
\begin{aligned}
& \phi^{(\nu, \mu)}(r, x+1)=\phi^{(\nu, \mu)}(r, x), \\
& \phi^{(\nu, \mu)}(r, x+\tau)=e^{-2 \pi i r} \phi^{(\nu, \mu)}(r, x), \\
& \phi^{(\nu, \mu)}(r, x+\eta)=e^{i \pi \eta(\mu-\nu)} \phi^{(\nu, \mu)}(r, x) .
\end{aligned}
$$

In order to fix the dependence of $\phi^{(\nu, \mu)}$ on $y=(s-t) / 2$, we note that it follows from $(3.55)-(3.58)$ that

$$
\begin{aligned}
& X_{k}^{l}(s+1, t-1)=X_{k}^{l}(s, t), \\
& X_{k}^{l}(s+\tau, t-\tau)=e^{-2 \pi i n \tau-2 \pi i n(s-t)+2 \pi i n \eta-2 \pi i \sum_{k} \xi_{k}} X_{k}^{l}(s, t),
\end{aligned}
$$

and, therefore,

$$
\begin{aligned}
& Y_{k}^{(\mu)}(y+1)=Y_{k}^{(\mu)}(y) \\
& Y_{k}^{(\mu)}(y+\tau)=e^{-\pi i N \tau-2 \pi i N y+\pi i N \eta-2 \pi i \sum_{k} \xi_{k}} Y_{k}^{(\mu)}(y) .
\end{aligned}
$$

This implies the following properties:

$$
\begin{aligned}
& \phi^{(\nu, \mu)}(r, x, y+1)=\phi^{(\nu, \mu)}(r, x, y), \\
& \phi^{(\nu, \mu)}(r, x, y+\tau)=e^{-\pi i N \tau-2 \pi i N y+\pi i N \eta-2 \pi i \sum_{k} \xi_{k}} \phi^{(\nu, \mu)}(r, x, y) .
\end{aligned}
$$


We also know that $\phi^{(\nu, \mu)}(r, x, y)$ is an entire function of $y$. Therefore, we conclude that it is a theta function of order $N$, i.e. it has $N$ zeros in the fundamental domain.

The above properties imply that the dependence on $x$ and $y$ factorizes and the function $\phi^{(\nu, \mu)}(\bar{v}, r, x, y)$ can be represented in the form

$$
\phi^{(\nu, \mu)}(\bar{v}, r, x, y)=\phi_{1}^{(\nu, \mu)}(r, x) \phi_{2}^{(\nu)}(\bar{v}, y),
$$

where the function $\phi_{1}^{(\nu, \mu)}(r, x)$ can be fixed by the following argument.

As it is seen from (5.30), the matrix elements of the matrix $T_{i j}^{(\nu \mu)}(r)$ are non-singular if $r=m \tau$ and

$$
\mu-\nu+2 m=Q k, \quad k \in \mathbb{Z}
$$

(and the matrix is non-degenerate) while for other integer values of $m$ the matrix elements have a simple pole at $r=m \tau$ and the matrix has the form of a matrix of rank 1 times a singular multiplier. The determinant of such matrix has a simple pole at $r=m \tau$. For even $Q$, the values of $m$ satisfying (5.48) are $m=\frac{1}{2}(Q k-\mu+\nu)(\mu-\nu$ is always even for even $Q$, see below) and we conjecture the following form of the function $\phi_{1}^{(\nu, \mu)}(r, x)$ which satisfies all the above properties:

$$
\phi_{1}^{(\nu, \mu)}(r, x)=\delta_{\mu, \nu(\bmod 2)} e^{\pi i(\mu-\nu) x} \frac{\theta_{1}(r \mid \tau) \theta_{1}(r+Q x / 2+(\mu-\nu) \tau / 2 \mid Q \tau / 2)}{\theta_{1}(Q x / 2 \mid Q \tau / 2) \theta_{1}(r+(\mu-\nu) \tau / 2 \mid Q \tau / 2)} .
$$

In fact the above transformation properties still hold if one multiplies $\phi_{1}^{(\nu, \mu)}(r, x)$ by any function of $r+(\mu-\nu) \tau / 2$. The choice of this function in (5.49) makes $Y_{k}^{(\nu, \mu)}(r, x)$ free of poles in $r$ and also free of zeros (except the zero at $r=-Q x / 2-(\mu-\nu) \tau / 2$ modulo the lattice spanned by $1, \tau)$. The delta-symbol $\delta_{\mu, \nu}(\bmod 2)$ comes from the selection rule (see section 5.7 below). Strictly speaking, the above transformation properties remain the same if one multiplies the right hand side of (5.49) by an elliptic function $f(x)$ of $x$ with periods $1, \tau$. However, it follows from the explicit form of $Y_{k}^{(\mu)}$ that the only poles of $\phi_{1}^{(\nu, \mu)}(r, x)$ are at the points $l \eta, l=0,1, \ldots, Q / 2-1$. But the right hand side of (5.49) already has poles at these points, so $f(x)$ must be free of poles and thus be a constant. For odd $Q$, the values of $m$ satisfying (5.48) are $m=Q k-(\mu-\nu)(Q+1) / 2, k \in \mathbb{Z}$ and we conjecture the following form of the function $\phi_{1}^{(\nu, \mu)}(r, x)$ which satisfies all the above properties:

$$
\phi_{1}^{(\nu, \mu)}(r, x)=e^{\pi i \ell_{\mu \nu} x} \frac{\theta_{1}(r \mid \tau) \theta_{1}\left(r+Q x+\ell_{\mu \nu} \tau / 2 \mid Q \tau\right)}{\theta_{1}(Q x \mid Q \tau) \theta_{1}\left(r+\ell_{\mu \nu} \tau / 2 \mid Q \tau\right)} .
$$

Here

$$
\ell_{\mu \nu}=\mu-\nu-\frac{1}{2}\left(1-(-1)^{\mu-\nu}\right) Q
$$

The formulas (5.49), (5.50) are also justified by computer calculations for $N=2$ and $N=4$. The function $\phi_{2}^{(\nu)}(\bar{v}, y)$ is not fixed. Finally, we note that numerical studies suggest the following dependence of $\phi_{2}^{(\nu)}(\bar{v}, y)$ on $y$ :

$$
\phi_{2}^{(\nu)}(\bar{v}, y)=\tilde{\phi}_{2}^{(\nu)}(\bar{v}) e^{2 \pi i \nu y} \prod_{k=1}^{n} \theta_{1}^{2}\left(y+v_{k} \mid \tau\right) .
$$




\subsection{The result for scalar products}

Now let us consider

$$
Y_{n+1}^{(\mu)}(r)=\sum_{l \in \mathbb{Z}_{Q}} \frac{\theta_{1}\left(l \eta+U_{n+1}+w_{0}\right)}{\theta_{1}(l \eta+x)} e^{-i l \pi \eta \mu}\left\langle\Psi_{\nu}(\bar{v}) \mid \Psi^{l}(\bar{u})\right\rangle,
$$

where $\bar{u}=\left\{u_{1}, \ldots, u_{n}\right\}$ are arbitrary parameters. ${ }^{2}$ We see that for the special choice of $w_{0}$,

$$
w_{0}=x-U_{n+1},
$$

$Y_{n+1}^{(\mu)}(r)$ equals the scalar product of the on-shell dual Bethe vector $\left\langle\Psi_{\nu}(\bar{v})\right|$ and the off-shell Bethe vector

$$
\left|\Psi_{\mu}(\bar{u})\right\rangle=\sum_{l \in \mathbb{Z}_{Q}} e^{-i l \pi \eta \mu}\left|\Psi^{l}(\bar{u})\right\rangle .
$$

Note that if we choose $w_{0}$ in this way, then

$$
r=\sum_{i=1}^{n}\left(v_{i}-u_{i}\right)
$$

According to (5.36), the scalar product has the form

$$
\left\langle\Psi_{\nu}(\bar{v}) \mid \Psi_{\mu}(\bar{u})\right\rangle=\phi_{1}^{(\nu, \mu)}(r, x) \phi_{2}^{(\nu)}(\bar{v}, y) \frac{\operatorname{det}_{1 \leq i, j \leq n} T_{i j}^{(\nu \mu)}(r)}{W_{n}(\bar{u}, \bar{v})},
$$

where

$$
W_{n}(\bar{u}, \bar{v})=\frac{\prod_{a<b}^{n} \theta_{1}\left(u_{a}-u_{b}\right) \theta_{1}\left(v_{b}-v_{a}\right)}{\prod_{p, q}^{n} \theta_{1}\left(u_{p}-v_{q}\right)}
$$

the matrix $T_{i j}^{(\nu \mu)}(r)$ is given by (5.29) and the function $\phi_{1}^{(\nu, \mu)}(r, x)$ by (5.49), (5.50). The function $\phi_{2}^{(\nu)}(\bar{v}, y)$ is still unknown.

\subsection{The selection rule}

We are going to show that if $\eta=2 P / Q$ with even $Q$ and odd $P$, then off-shell Bethe vectors $\left\langle\Psi_{\nu}(\bar{v})\right|$ and $\left|\Psi_{\mu}(\bar{u})\right\rangle$ with $\mu-\nu=1(\bmod 2)$ are orthogonal: $\left\langle\Psi_{\nu}(\bar{v}) \mid \Psi_{\mu}(\bar{u})\right\rangle=0$. This follows from the fact that the off-shell Bethe vectors are eigenvectors of the operator $U_{3}$ :

$$
\mathrm{U}_{3}\left|\Psi_{\mu}(\bar{u})\right\rangle=(-1)^{\mu+n}\left|\Psi_{\mu}(\bar{u})\right\rangle, \quad\left\langle\Psi_{\nu}(\bar{v})\right| \mathrm{U}_{3}=(-1)^{\nu+n}\left\langle\Psi_{\nu}(\bar{v})\right| .
$$

Indeed, computing the matrix element $\left\langle\Psi_{\nu}(\bar{v})\left|\mathrm{U}_{3}\right| \Psi_{\mu}(\bar{u})\right\rangle$ in two ways (acting by $\mathrm{U}_{3}$ to the right and to the left), we get:

$$
\left((-1)^{\mu+n}-(-1)^{\nu+n}\right)\left\langle\Psi_{\nu}(\bar{v}) \mid \Psi_{\mu}(\bar{u})\right\rangle=0
$$

which means that $\left\langle\Psi_{\nu}(\bar{v}) \mid \Psi_{\mu}(\bar{u})\right\rangle=0$ if $\mu-\nu=1(\bmod 2)$.

\footnotetext{
${ }^{2}$ We draw the reader's attention to the fact that now the set $\bar{u}$ consists only of $n$ parameters: $\bar{u}=$ $\left\{u_{1}, \ldots, u_{n}\right\}$. The auxiliary parameter $u_{n+1}$ is no longer required, and we excluded it for the sake of simplification of notation.
} 
To prove (5.56), we use the result of section 3.4 that the action of $U_{3}$ to the vectors $\left|\Psi^{l}\right\rangle$ and $\left\langle\Psi^{l}\right|$ is equivalent to the shift of $s, t$ by 1 and the fact that $\left|\Psi_{\mu}(s+2, t+2)\right\rangle=\left|\Psi_{\mu}(s, t)\right\rangle$. We have:

$$
\begin{aligned}
\left|\Psi_{\mu}(s, t)\right\rangle= & \sum_{l \in \mathbb{Z}_{Q}} e^{-2 \pi i l P \mu / Q}\left|\Psi^{l}(s, t)\right\rangle \\
= & \sum_{l=0}^{Q-1} e^{-2 \pi i l P \mu / Q}\left|\Psi^{0}\left(s+\frac{2 P l}{Q}, t+\frac{2 P l}{Q}\right)\right\rangle \\
= & \sum_{l=0}^{Q / 2-1} e^{-2 \pi i l P \mu / Q}\left|\Psi^{0}\left(s+\frac{2 P l}{Q}, t+\frac{2 P l}{Q}\right)\right\rangle \\
& +\sum_{l=Q / 2}^{Q-1} e^{-2 \pi i l P \mu / Q}\left|\Psi^{0}\left(s+\frac{2 P l}{Q}, t+\frac{2 P l}{Q}\right)\right\rangle \\
& Q / 2-1 \\
& \sum_{l=0} e^{-2 \pi i l P \mu / Q}\left|\Psi^{0}\left(s+\frac{2 P l}{Q}, t+\frac{2 P l}{Q}\right)\right\rangle \\
& +(-1)^{\mu} \sum_{l=0}^{Q / 2-1} e^{-2 \pi i l P \mu / Q}\left|\Psi^{0}\left(s+\frac{2 P l}{Q}+1, t+\frac{2 P l}{Q}+1\right)\right\rangle \\
= & \left(1+(-1)^{\mu+n} \mathrm{U}_{3}\right) \sum_{l=0}^{Q / 2-1} e^{-2 \pi i l P \mu / Q}\left|\Psi^{0}\left(s+\frac{2 P l}{Q}, t+\frac{2 P l}{Q}\right)\right\rangle,
\end{aligned}
$$

from which the first relation in (5.56) follows (recall that $\mathrm{U}_{3}^{2}=1$ ). The argument for the dual vector $\left\langle\Psi_{\nu}\right|$ is the same. Note that the selection rule is valid also for $Y_{k}^{(\nu, \mu)}$ : it vanishes for $\mu-\nu=1(\bmod 2)$ (the proof is similar).

However, if $Q$ is odd, then (5.56) does not hold in general and the selection rule does not work.

\subsection{Orthogonality and norm of on-shell Bethe vectors}

Let us show that if the vector $\left|\Psi_{\mu}(\bar{u})\right\rangle$ is on-shell and the sets $\bar{u}$ and $\bar{v}$ do not coincide, the scalar product (5.54) vanishes. We should show that the matrix $T_{i k}^{(\nu \mu)}(r)(5.30)$ becomes degenerate if the parameters $\bar{u}$ satisfy the Bethe equations (3.25), which we write here in the form

$$
e^{2 i \pi \eta \mu} \frac{a\left(u_{j}\right)}{d\left(u_{j}\right)}=(-1)^{n-1} \frac{h\left(u_{j}, \bar{u}\right)}{h\left(\bar{u}, u_{j}\right)} .
$$

We are going to show that the rows of the matrix $T_{i k}^{(\nu \mu)}(r)$ are linearly dependent. Set

$$
x_{j}=\frac{g\left(v_{j}, \bar{v}_{j}\right)}{g\left(v_{j}, \bar{u}\right)} .
$$

Note that since the sets $\bar{u}, \bar{v}$ do not coincide, there is at least one non-vanishing $x_{j}$. Consider a linear combination

$$
X=\sum_{i} x_{i} T_{i k}^{(\nu \mu)}(r)=a\left(u_{k}\right) f\left(\bar{v}, u_{k}\right) E^{+}+d\left(u_{k}\right) f\left(u_{k}, \bar{v}\right) E^{-},
$$


where

$$
E^{ \pm}=\frac{\theta_{1}^{\prime}(0)}{\theta_{1}(r)} \sum_{i} x_{i}\left(e^{ \pm i \pi \eta \nu} \frac{\theta_{1}\left(v_{i}-u_{k}-r\right)}{\theta_{1}\left(v_{i}-u_{k}\right)}-e^{ \pm i \pi \eta \mu} \frac{\theta_{1}\left(v_{i}-u_{k} \pm \eta-r\right)}{\theta_{1}\left(v_{i}-u_{k} \pm \eta\right)}\right) .
$$

In order to compute $E^{ \pm}$, we consider an auxiliary contour integral

$$
I^{ \pm}=\frac{\theta_{1}^{\prime}(0)}{\theta_{1}(r)} \oint \frac{d z}{2 \pi i}\left(e^{ \pm i \pi \eta \nu} \frac{\theta_{1}\left(z-u_{k}-r\right)}{\theta_{1}\left(z-u_{k}\right)}-e^{ \pm i \pi \eta \mu} \frac{\theta_{1}\left(z-u_{k} \pm \eta-r\right)}{\theta_{1}\left(z-u_{k} \pm \eta\right)}\right) \prod_{a=1}^{n} \frac{\theta_{1}\left(z-u_{a}\right)}{\theta_{1}\left(z-v_{a}\right)} .
$$

The integral is taken along the boundary of the fundamental parallelogram spanned by $1, \tau$. Since $r=\sum_{i} v_{i}-\sum_{i} u_{i}$ (see (5.53)), it is easy to see that the integrand is doubleperiodic with periods $1, \tau$. Therefore, $I^{ \pm}=0$. On the other hand, the integral can be calculated as sum of the residues inside the fundamental parallelogram. The sum of the residues at the poles at $z=v_{j}$ gives $E^{ \pm}$. One more contribution comes from the simple pole at $z-u_{k} \pm \eta=0$. Thus we arrive at

$$
E^{ \pm}=-e^{ \pm i \pi \eta \mu} \theta_{1}^{\prime}(0) \prod_{a=1}^{n} \frac{\theta_{1}\left(u_{k}-u_{a} \mp \eta\right)}{\theta_{1}\left(u_{k}-v_{a} \mp \eta\right)}
$$

or

$$
E^{+}=-e^{i \pi \eta \mu} \theta_{1}^{\prime}(0) \frac{h\left(\bar{u}, u_{k}\right)}{h\left(\bar{v}, u_{k}\right)}, \quad E^{-}=-e^{-i \pi \eta \mu} \theta_{1}^{\prime}(0) \frac{h\left(u_{k}, \bar{u}\right)}{h\left(u_{k}, \bar{v}\right)} .
$$

Substituting these results into (5.59), we obtain:

$$
X=-\theta_{1}^{\prime}(0) g\left(u_{k}, \bar{v}\right)\left[(-1)^{n} e^{i \pi \eta \mu} a\left(u_{k}\right) h\left(\bar{u}, u_{k}\right)+e^{-i \pi \eta \mu} d\left(u_{k}\right) h\left(u_{k}, \bar{u}\right)\right]
$$

which is equal to 0 due to the Bethe equations (5.57). Thus the rows of the matrix $T_{i k}^{(\nu \mu)}(r)$ are linearly dependent and hence $\operatorname{det} T_{i k}^{(\nu \mu)}(r)=0$.

Note that we did not use the fact that the set $\bar{v}$ satisfies the Bethe equations. Therefore, $\operatorname{det} T_{i k}^{(\nu \mu)}(r)$ vanishes when the following weaker conditions are fulfilled: i) the set $\bar{u}$ satisfies the Bethe equations, ii) $r=\sum_{i}\left(v_{i}-u_{i}\right)$, iii) the sets $\bar{u}, \bar{v}$ do not coincide.

The square of the norm of the vector $\left|\Psi_{\nu}(\bar{v})\right\rangle$ can be obtained in the $\operatorname{limit}^{3} u_{i} \rightarrow v_{i}$. We set $u_{i}=v_{i}+\varepsilon$ in equation (5.54), so that $r=-n \varepsilon$, and tend $\varepsilon \rightarrow 0$. The matrix $T_{i k}^{(\nu \nu)}(r)$ becomes singular but the factor $W(\bar{u}, \bar{v})$ in the denominator brings the multiplier $\theta_{1}^{n}(\varepsilon)$ which cancels the singularity. The limiting procedure is straightforward and the result is ${ }^{4}$

$$
\begin{aligned}
& \lim _{\varepsilon \rightarrow 0}\left(\theta_{1}(\varepsilon) T_{i k}^{(\nu \nu)}(-n \varepsilon)\right)=\theta_{1}(\eta) e^{-i \pi \eta \nu} d\left(v_{k}\right) f\left(v_{k}, \bar{v}_{k}\right) K\left(v_{i}-v_{k}\right), \quad i \neq k, \\
& \lim _{\varepsilon \rightarrow 0}\left(\theta_{1}(\varepsilon) T_{i i}^{(\nu \nu)}(-n \varepsilon)\right)=-\theta_{1}(\eta) e^{-i \pi \eta \nu} d\left(v_{i}\right) f\left(v_{i}, \bar{v}_{i}\right)\left(\partial_{v_{i}} \log \frac{a\left(v_{i}\right)}{d\left(v_{i}\right)}+\sum_{j \neq i} K\left(v_{i}-v_{j}\right)\right),
\end{aligned}
$$

where

$$
K(u)=\frac{\theta_{1}^{\prime}(u-\eta)}{\theta_{1}(u-\eta)}-\frac{\theta_{1}^{\prime}(u+\eta)}{\theta_{1}(u+\eta)} .
$$

\footnotetext{
${ }^{3}$ See remark in the beginning of section 3 .

${ }^{4}$ The same result is reproduced for the limit $u_{i}=v_{i}+\varepsilon_{i}, \varepsilon_{i} \rightarrow 0$.
} 
Therefore,

$$
\left\langle\Psi_{\nu}(\bar{v}) \mid \Psi_{\nu}(\bar{v})\right\rangle=\phi_{1}^{(\nu, \nu)}(0, x) \phi_{2}^{(\nu)}(\bar{v}, y) \theta_{1}^{n}(\eta) e^{-i n \pi \eta \nu} d(\bar{v}) \prod_{a \neq b}^{n} f\left(v_{a}, v_{b}\right) \operatorname{det}_{1 \leq i, k \leq n} G_{i k},
$$

where

$$
\begin{aligned}
G_{i k} & =-\delta_{i k}\left(\partial_{v_{i}} \log \frac{a\left(v_{i}\right)}{d\left(v_{i}\right)}+\sum_{j=1}^{n} K\left(v_{i}-v_{j}\right)\right)+K\left(v_{i}-v_{k}\right), \\
\phi_{1}^{(\nu, \nu)}(0, x) & = \begin{cases}\frac{\theta_{1}^{\prime}(0 \mid \tau)}{\theta_{1}^{\prime}(0 \mid Q \tau / 2)} & \text { for } Q \text { even, } \\
\frac{\theta_{1}^{\prime}(0 \mid \tau)}{\theta_{1}^{\prime}(0 \mid Q \tau)} & \text { for } Q \text { odd. }\end{cases}
\end{aligned}
$$

and similarly to $(5.3)$

$$
d(\bar{v})=\prod_{i=1}^{n} d\left(v_{i}\right) .
$$

This is the elliptic version of the Gaudin's formula [41] and $G_{i k}$ is the elliptic analogue of the Gaudin's matrix. If we take logarithm of the Bethe equations, denote

$$
B_{j}=-\log \frac{a\left(v_{j}\right)}{d\left(v_{j}\right)}+\log \frac{f\left(v_{j}, \bar{v}\right)}{f\left(\bar{v}, v_{j}\right)}-2 \pi i \eta \nu
$$

(so that the Bethe equations read $B_{j}=2 \pi i n_{j}, n_{j} \in \mathbb{Z}$ ), then $G_{j k}=\partial B_{j} / \partial v_{k}$.

However, in the case of the norm the result (5.61) is somewhat meaningless because it is multiplied by an unknown function $\phi_{2}$ of $\bar{v}$.

\subsection{Normalized scalar products}

The unknown function $\phi_{2}$ does not enter the specially normalized scalar products

$$
S_{\nu \mu}(\bar{v}, \bar{u})=\frac{\left\langle\Psi_{\nu}(\bar{v}) \mid \Psi_{\mu}(\bar{u})\right\rangle}{\left\langle\Psi_{\nu}(\bar{v}) \mid \Psi_{\nu}(\bar{v})\right\rangle}
$$

where the vector $\left\langle\Psi_{\nu}(\bar{v})\right|$ is an on-shell vector and $\left|\Psi_{\mu}(\bar{u})\right\rangle$ is an arbitrary of-shell Bethe vector. It is easy to see that (5.65) differs from the usual normalized scalar product, in which the denominator would contain the norms of both vectors. Nevertheless, in the models with the 6 -vertex $R$-matrix, it is normalization (5.65) that is sufficient to calculate the form factors of local operators and correlation functions (see the more detailed discussion of this issue in section 6). It is for this reason that we are interested in such a special normalization.

Collecting the formulas obtained above together, we arrive at the following result for these scalar products:

$$
S_{\nu \mu}(\bar{v}, \bar{u})=e^{\pi i \eta n \nu} \prod_{p, q} \frac{\theta_{1}\left(u_{p}-v_{q}\right)}{\theta_{1}\left(v_{p}-v_{q}+\eta\right)} \prod_{a<b} \frac{\theta_{1}\left(v_{a}-v_{b}\right)}{\theta_{1}\left(u_{a}-u_{b}\right)} \frac{\phi_{1}^{(\nu, \mu)}(r, x)}{\phi_{1}^{(\nu, \nu)}(0, x)} \frac{\operatorname{det}_{n \times n} T_{j k}^{(\nu \mu)}(r)}{d(\bar{v}) \operatorname{det}_{n \times n} G_{j k}},
$$


where $r=\sum_{i} v_{i}-\sum_{i} u_{i}$, the matrices $T_{j k}^{(\nu \mu)}(r), G_{j k}$ are given by formulas (5.30), (5.62) respectively, $d(\bar{v})$ by (5.64) and the functions $\phi_{1}^{(\nu, \mu)}(r, x), \phi_{1}^{(\nu, \nu)}(0, x)$ by $(5.49),(5.50),(5.63)$. Note that at $\mu=\nu$ and $\sum_{i} u_{i}=\sum_{i} v_{i}$ this formula becomes

$$
S_{\nu \nu}(\bar{v}, \bar{u})=e^{\pi i \eta n \nu} \prod_{p, q} \frac{\theta_{1}\left(u_{p}-v_{q}\right)}{\theta_{1}\left(v_{p}-v_{q}+\eta\right)} \prod_{a<b} \frac{\theta_{1}\left(v_{a}-v_{b}\right)}{\theta_{1}\left(u_{a}-u_{b}\right)} \frac{\operatorname{det}_{n \times n}\left(\partial T_{\nu}\left(u_{k}, \bar{v}\right) / \partial v_{j}\right)}{d(\bar{v}) \operatorname{det}_{n \times n} G_{j k}},
$$

which resembles the result for the $X X Z$ case [36].

\section{Concluding remarks}

We have obtained the determinant representation (5.66) for the specially normalized scalar products of Bethe vectors (5.65) in the inhomogeneous 8-vertex model (or equivalently, in the inhomogeneous $X Y Z$ spin- $\frac{1}{2}$ chain) in the case when the anisotropy parameter $\eta$ is a rational number $\eta=2 P / Q$. Recall, however, that one can take the homogeneous limit in all our formulas. The matrix $T_{j k}^{(\nu \mu)}(r)$ in (5.66) is given by (5.29) or (5.30). Note that this matrix is essentially the same as the matrix entering the determinant representation for scalar products of Bethe vectors in the elliptic cyclic SOS model obtained in [48].

A more general case when the Bethe vectors are well-defined is the case when $\eta$ is a point of finite order on the elliptic curve, i.e., $Q \eta=2 P_{1}+P_{2} \tau$ with some integer $Q, P_{1}, P_{2}$. We hope that it is not too difficult to extend our results to this case. Other possible generalizations of our results are related to the scalar products of Bethe vectors in the 8vertex model with twisted boundary conditions [68] (in the 8-vertex case the only possible twist matrices are the Pauli matrices) and in the $X Y Z$ spin chain with higher spin [13, 20].

We also did not consider the case of scalar products in which the right and left vectors depend on a different number of parameters. Note that the derivation of the system of linear equations for this case remains exactly the same. However, presumably, in this case, the resulting system has only trivial solutions.

A comment on the limit to the $X X Z$ case (the 6 -vertex model) is in order. Formally, this is the limit $\tau \rightarrow+i \infty$ when the Jacobi theta functions tend to trigonometric functions. However, as is seen from (5.66), (5.30), the limit of our result does not coincide with the well known answer for the $X X Z$ case [43]. The reason is in the different structure of the off-shell Bethe vectors: the trigonometric limit of the off-shell Bethe vectors constructed in the framework of the generalized algebraic Bethe ansatz method differs from off-shell Bethe vectors usually considered in the $X X Z$ type models. It is enough to say that our off-shell vectors essentially depend on the auxiliary parameters $s, t$ which are absent in the standard algebraic Bethe ansatz approach.

One of the most attractive areas is the application of the obtained result (5.66) to the calculation of form factors and correlation functions. However, for this it is necessary to obtain formulas for the action of the monodromy matrix entries on Bethe vectors. In models with the 6 -vertex $R$-matrix, such formulas are well known. Schematically, they can be represented in the form

$$
\mathcal{T}_{a b}(z)|\Psi(\bar{v})\rangle=\sum_{\bar{v}^{\prime}} t_{a b}\left(\bar{v}^{\prime}\right)\left|\Psi\left(\bar{v}^{\prime}\right)\right\rangle,
$$


where $\bar{v}^{\prime}$ is a subset of $\bar{v} \cup z$, and $t_{a b}\left(\bar{v}^{\prime}\right)$ are some numerical coefficients. The sum in (6.1) is taken with respect to all possible subsets of fixed cardinality. The latter depends on the concrete matrix element $\mathcal{T}_{a b}$.

If we assume that similar action formulas also exist in the 8-vertex model, then we immediately get access to the form factors of local spin operators. Indeed, the latter can be expressed through the elements of the quantum monodromy matrix using the formulas of the inverse scattering problem $[37,38]$ :

$$
E_{m}^{i j}=\left(\prod_{k=1}^{m-1} \mathrm{~T}\left(\xi_{k}\right)\right) \mathcal{T}_{j i}\left(\xi_{m}\right)\left(\prod_{k=1}^{m} \mathrm{~T}\left(\xi_{k}\right)\right)^{-1} .
$$

Here $E_{m}^{i j}$ is an elementary unit matrix acting in the $m$ th local quantum space $V_{m}$. Therefore, all form factors of local operators reduce to form factors of the entries of the quantum monodromy matrix. For instance, magnetization $\left\langle\frac{1}{2}\left(1-\sigma_{3}^{(m)}\right)\right\rangle$ is given by

$$
\frac{\left\langle\Psi_{\nu}(\bar{v})\left|\frac{1}{2}\left(1-\sigma_{3}^{(m)}\right)\right| \Psi_{\nu}(\bar{v})\right\rangle}{\left\langle\Psi_{\nu}(\bar{v}) \mid \Psi_{\nu}(\bar{v})\right\rangle}=\frac{\left\langle\Psi_{\nu}(\bar{v})\left|D\left(\xi_{m}\right)\right| \Psi_{\nu}(\bar{v})\right\rangle}{T_{\nu}\left(\xi_{m} \mid \bar{v}\right)\left\langle\Psi_{\nu}(\bar{v}) \mid \Psi_{\nu}(\bar{v})\right\rangle},
$$

where $T_{\nu}\left(\xi_{m} \mid \bar{v}\right)$ is the eigenvalue of $\mathbf{T}\left(\xi_{m}\right)$ on $\left|\Psi_{\nu}(\bar{v})\right\rangle$.

If the action of the operator $D\left(\xi_{m}\right)$ on the vector $\left|\Psi_{\nu}(\bar{v})\right\rangle$ is given by a linear combination of off-shell vectors similar to (6.1), then this form factor reduces to scalar products (5.66). Other form factors are calculated similarly.

However, we would like to emphasize that the assumption of the existence of the action formula similar to (6.1) in the models with the 8-vertex $R$-matrix is rather strong assumption. For the moment, there is no evidence that such a formula does exist. We are going to highlight this issue in our forthcoming publications.

\section{Acknowledgments}

We thank A. Liashyk for useful comments and discussions. This work is supported by the Russian Science Foundation under grant 19-11-00062 and performed in Steklov Mathematical Institute of Russian Academy of Sciences.

\section{A Null-vector}

As one of the applications of the obtained formulas, we show that the scalar products $\left\langle\Psi_{\nu}(\bar{v}) \mid \Psi_{\mu}(\bar{u})\right\rangle$ vanish if the set $\bar{u}$ contains $\xi_{p}$ and $\xi_{p}-\eta$ for some $p=1, \ldots, N$. (We denote them as $u_{1}=\xi_{p}$ and $u_{2}=\xi_{p}-\eta$, so that $d\left(u_{1}\right)=a\left(u_{2}\right)=0$.) To see this, we will show that in this case $\operatorname{det} T_{j k}^{(\nu \mu)}=0$. Indeed, from the formula (5.30) we conclude that the first column is

$$
T_{j 1}^{(\nu \mu)}=\frac{\theta_{1}^{\prime}(0)}{\theta_{1}(r)} a\left(\xi_{p}\right) f\left(\bar{v}, \xi_{p}\right)\left(e^{i \pi \eta \nu} \frac{\theta_{1}\left(\xi_{p}-v_{j}+r\right)}{\theta_{1}\left(\xi_{p}-v_{j}\right)}-e^{i \pi \eta \mu} \frac{\theta_{1}\left(\xi_{p}-v_{j}-\eta+r\right)}{\theta_{1}\left(\xi_{p}-v_{j}-\eta\right)}\right)
$$


and the second column is

$$
\begin{aligned}
T_{j 2}^{(\nu \mu)}=\frac{\theta_{1}^{\prime}(0)}{\theta_{1}(r)} d\left(\xi_{p}-\eta\right) f\left(\xi_{p}-\eta, \bar{v}\right) & \\
& \times\left(e^{-i \pi \eta \nu} \frac{\theta_{1}\left(\xi_{p}-\eta-v_{j}+r\right)}{\theta_{1}\left(\xi_{p}-\eta-v_{j}\right)}-e^{-i \pi \eta \mu} \frac{\theta_{1}\left(\xi_{p}-v_{j}+r\right)}{\theta_{1}\left(\xi_{p}-v_{j}\right)}\right) \\
= & \frac{\theta_{1}^{\prime}(0)}{\theta_{1}(r)} e^{-\pi i \eta(\mu+\nu)} d\left(\xi_{p}-\eta\right) f\left(\xi_{p}-\eta, \bar{v}\right) \\
& \times\left(e^{i \pi \eta \mu} \frac{\theta_{1}\left(\xi_{p}-\eta-v_{j}+r\right)}{\theta_{1}\left(\xi_{p}-\eta-v_{j}\right)}-e^{i \pi \eta \nu} \frac{\theta_{1}\left(\xi_{p}-v_{j}+r\right)}{\theta_{1}\left(\xi_{p}-v_{j}\right)}\right) .
\end{aligned}
$$

We see that the two columns are proportional to each other and hence the determinant vanishes.

If the set of dual eigenvectors $\left\langle\Psi_{\nu}(\bar{v})\right|$ is complete (which is usually believed), this result means that the vector $\left|\Psi_{\mu}(\bar{u})\right\rangle$ in which $u_{1}=\xi_{p}, u_{2}=\xi_{p}-\eta$ is a null-vector.

\section{B The case $N=2$}

The case $N=2$ is relatively simple but instructive. In this case there is only one Bethe equation for the Bethe root $v$ of the form

$$
e^{2 \pi i \eta \nu} \frac{\theta_{1}\left(v-\xi_{1}+\eta\right) \theta_{1}\left(v-\xi_{2}+\eta\right)}{\theta_{1}\left(v-\xi_{1}\right) \theta_{1}\left(v-\xi_{2}\right)}=1 .
$$

This equation has 4 solutions in the fundamental domain (the number of solutions is equal to the dimension of the quantum space):

$$
v=\frac{1}{2}\left(\xi_{1}+\xi_{2}-\eta\right)+\omega
$$

where $\omega$ is a half-period: $\omega=0,1 / 2$ (in these cases $\nu=0$ ) and $\omega=\tau / 2,(\tau+1) / 2$ (in these cases $\nu=1)$. Note that this agrees with the sum rule (4.24).

Diagonalization of the transfer matrix. For brevity, we denote $a_{1}=a^{8 \mathrm{v}}\left(u-\xi_{1}\right)$, $a_{2}=a^{8 \mathrm{v}}\left(u-\xi_{2}\right), b_{1}=b^{8 \mathrm{v}}\left(u-\xi_{1}\right)$, etc. In the natural basis $|++\rangle,|+-\rangle,|-+\rangle,|--\rangle$ the transfer matrix of the model is given by

$$
\mathrm{T}(u)=\left(\begin{array}{cccc}
a_{1} a_{2}+b_{1} b_{2} & 0 & 0 & c_{1} d_{2}+d_{1} c_{2} \\
0 & a_{1} b_{2}+b_{1} a_{2} & c_{1} c_{2}+d_{1} d_{2} & 0 \\
0 & c_{1} c_{2}+d_{1} d_{2} & a_{1} b_{2}+b_{1} a_{2} & 0 \\
c_{1} d_{2}+d_{1} c_{2} & 0 & 0 & a_{1} a_{2}+b_{1} b_{2}
\end{array}\right) .
$$

This matrix can be easily diagonalized by hands. The result is:

\begin{tabular}{|c|c|c|c|}
\hline & Eigenvector & Eigenvalue & Bethe root \\
\hline 1$)$ & $|+-\rangle-|-+\rangle$ & $a_{1} b_{2}+b_{1} a_{2}-c_{1} c_{2}-d_{1} d_{2}$ & $v_{1}=\frac{1}{2}\left(\xi_{1}+\xi_{2}-\eta\right), \nu=0$ \\
\hline 2$)$ & $|+-\rangle+|-+\rangle$ & $a_{1} b_{2}+b_{1} a_{2}+c_{1} c_{2}+d_{1} d_{2}$ & $v_{2}=\frac{1}{2}\left(\xi_{1}+\xi_{2}-\eta\right)+\frac{1}{2}, \nu=0$ \\
\hline 3$)$ & $|++\rangle+|--\rangle$ & $a_{1} a_{2}+b_{1} b_{2}+c_{1} d_{2}+d_{1} c_{2}$ & $v_{3}=\frac{1}{2}\left(\xi_{1}+\xi_{2}-\eta\right)+\frac{\tau+1}{2}, \nu=1$ \\
\hline 4$)$ & $|++\rangle-|--\rangle$ & $a_{1} a_{2}+b_{1} b_{2}-c_{1} d_{2}-d_{1} c_{2}$ & $v_{4}=\frac{1}{2}\left(\xi_{1}+\xi_{2}-\eta\right)+\frac{\tau}{2}, \nu=1$ \\
\hline
\end{tabular}


Similar results hold for left eigenvectors. After some transformations the eigenvalues can be brought to the form

$$
\begin{gathered}
T_{0}\left(u ; v_{1}\right)=\frac{2}{\theta_{2}^{2}(0) \theta_{3}(0) \theta_{4}(0)}\left[\theta_{4}(0) \theta_{3}(\eta) \theta_{3}\left(\frac{\xi_{1}-\xi_{2}+\eta}{2}\right) \theta_{3}\left(\frac{\xi_{1}-\xi_{2}-\eta}{2}\right) \theta_{4}^{2}\left(u-\frac{\xi_{1}+\xi_{2}-\eta}{2}\right)\right. \\
\left.-\theta_{3}(0) \theta_{4}(\eta) \theta_{4}\left(\frac{\xi_{1}-\xi_{2}+\eta}{2}\right) \theta_{4}\left(\frac{\xi_{1}-\xi_{2}-\eta}{2}\right) \theta_{3}^{2}\left(u-\frac{\xi_{1}+\xi_{2}-\eta}{2}\right)\right], \\
T_{0}\left(u ; v_{2}\right)=\frac{2}{\theta_{2}^{2}(0) \theta_{3}(0) \theta_{4}(0)}\left[\theta_{3}(0) \theta_{4}(\eta) \theta_{3}\left(\frac{\xi_{1}-\xi_{2}+\eta}{2}\right) \theta_{3}\left(\frac{\xi_{1}-\xi_{2}-\eta}{2}\right) \theta_{4}^{2}\left(u-\frac{\xi_{1}+\xi_{2}-\eta}{2}\right)\right. \\
\left.-\theta_{4}(0) \theta_{3}(\eta) \theta_{4}\left(\frac{\xi_{1}-\xi_{2}+\eta}{2}\right) \theta_{4}\left(\frac{\xi_{1}-\xi_{2}-\eta}{2}\right) \theta_{3}^{2}\left(u-\frac{\xi_{1}+\xi_{2}-\eta}{2}\right)\right], \\
T_{1}\left(u ; v_{3}\right)=\frac{\left.2-\xi_{2}\right)}{\theta_{2}^{2}(0) \theta_{3}(0) \theta_{4}(0)}\left[\theta_{3}(0) \theta_{4}(\eta) \theta_{2}\left(\frac{\xi_{1}-\xi_{2}+\eta}{2}\right) \theta_{2}\left(\frac{\xi_{1}-\xi_{2}-\eta}{2}\right) \theta_{1}^{2}\left(u-\frac{\xi_{1}+\xi_{2}-\eta}{2}\right)\right. \\
\left.-\theta_{4}(0) \theta_{3}(\eta) \theta_{1}\left(\frac{\xi_{1}-\xi_{2}+\eta}{2}\right) \theta_{1}\left(\frac{\xi_{1}-\xi_{2}-\eta}{2}\right) \theta_{2}^{2}\left(u-\frac{\xi_{1}+\xi_{2}-\eta}{2}\right)\right], \\
T_{1}\left(u ; v_{4}\right)=\frac{\left.2-\xi_{1}\right)}{\theta_{2}^{2}(0) \theta_{3}(0) \theta_{4}(0)}\left[\theta_{4}(0) \theta_{3}(\eta) \theta_{2}\left(\frac{\xi_{1}-\xi_{2}+\eta}{2}\right) \theta_{2}\left(\frac{\xi_{1}-\xi_{2}-\eta}{2}\right) \theta_{1}^{2}\left(u-\frac{\xi_{1}+\xi_{2}-\eta}{2}\right)\right. \\
\left.-\theta_{3}(0) \theta_{4}(\eta) \theta_{1}\left(\frac{\xi_{1}-\xi_{2}+\eta}{2}\right) \theta_{1}\left(\frac{\xi_{1}-\xi_{2}-\eta}{2}\right) \theta_{2}^{2}\left(u-\frac{\xi_{1}+\xi_{2}-\eta}{2}\right)\right] .
\end{gathered}
$$

It can be shown by a straightforward calculation that this form is the same as the expression (5.7):

$$
\begin{aligned}
T_{\nu}\left(u ; v_{a}\right)= & e^{\pi i \eta \nu} \theta_{1}\left(u-\xi_{1}+\eta\right) \theta_{1}\left(u-\xi_{2}+\eta\right) \frac{\theta_{1}\left(u-v_{a}-\eta\right)}{\theta_{1}\left(u-v_{a}\right)} \\
& +e^{-\pi i \eta \nu} \theta_{1}\left(u-\xi_{1}\right) \theta_{1}\left(u-\xi_{2}\right) \frac{\theta_{1}\left(u-v_{a}+\eta\right)}{\theta_{1}\left(u-v_{a}\right)}
\end{aligned}
$$

for $a=1, \ldots, 4$.

The Bethe vectors. For illustrative purposes, we give here the explicit expression for the (off-shell) Bethe vectors. We recall that

$$
\begin{aligned}
\mu(u ; s, t) & =\theta_{1}\left(\frac{1}{2}(s-t)+u \mid \tau\right), \\
\gamma_{k} & =\frac{1}{\theta_{2}\left(\tau_{k} \mid \tau\right)}, \quad \tau_{k}=\frac{1}{2}\left(s_{k}+t_{k}\right), \quad s_{k}=s+k \eta, \quad t_{k}=t+k \eta .
\end{aligned}
$$

The right vacuum is

$$
\left|\Omega^{l-1}\right\rangle=\left(\begin{array}{c}
\theta_{1}\left(s_{l-1}+\xi_{1} \mid 2 \tau\right) \\
\theta_{4}\left(s_{l-1}+\xi_{1} \mid 2 \tau\right)
\end{array}\right) \otimes\left(\begin{array}{c}
\theta_{1}\left(s_{l}+\xi_{2} \mid 2 \tau\right) \\
\theta_{4}\left(s_{l}+\xi_{2} \mid 2 \tau\right)
\end{array}\right) .
$$

Let us denote the basis vectors as

$$
\begin{array}{rlrl}
\left|e_{1}\right\rangle & =\frac{1}{2}(|+-\rangle-|-+\rangle), & \left|e_{2}\right\rangle & =\frac{1}{2}(|+-\rangle+|-+\rangle), \\
\left|e_{3}\right\rangle & =\frac{1}{2}(|++\rangle-|--\rangle), & \left|e_{4}\right\rangle=\frac{1}{2}(|++\rangle+|--\rangle) .
\end{array}
$$


For the vector

$$
\left|\Psi^{l}(u)\right\rangle=B_{l-1, l+1}(u)\left|\Omega^{l-1}\right\rangle
$$

we have

$$
\left|\Psi^{l}(u)\right\rangle=A_{1}^{(l)}\left|e_{1}\right\rangle+A_{2}^{(l)}\left|e_{2}\right\rangle+A_{3}^{(l)}\left|e_{3}\right\rangle+A_{4}^{(l)}\left|e_{4}\right\rangle,
$$

where the coefficients $A_{i}^{(l)}$ read:

$$
\begin{aligned}
& A_{1}^{(l)}=\frac{\gamma_{l-1} \gamma_{l+1}}{\mu(u ; s, t)}\left[\left(\theta_{4}\left(t_{l-1}-u \mid 2 \tau\right) \theta_{1}\left(t_{l+1}-u \mid 2 \tau\right) \theta_{1}\left(s_{l-1}+\xi_{1} \mid 2 \tau\right) \theta_{4}\left(s_{l}+\xi_{2} \mid 2 \tau\right)\right.\right. \\
& \left.+\theta_{1}\left(t_{l-1}-u \mid 2 \tau\right) \theta_{4}\left(t_{l+1}-u \mid 2 \tau\right) \theta_{4}\left(s_{l-1}+\xi_{1} \mid 2 \tau\right) \theta_{1}\left(s_{l}+\xi_{2} \mid 2 \tau\right)\right)\left(a_{1} b_{2}-c_{1} c_{2}\right) \\
& +\left(\theta_{4}\left(t_{l-1}-u \mid 2 \tau\right) \theta_{1}\left(t_{l+1}-u \mid 2 \tau\right) \theta_{4}\left(s_{l-1}+\xi_{1} \mid 2 \tau\right) \theta_{1}\left(s_{l}+\xi_{2} \mid 2 \tau\right)\right. \\
& \left.+\theta_{1}\left(t_{l-1}-u \mid 2 \tau\right) \theta_{4}\left(t_{l+1}-u \mid 2 \tau\right) \theta_{1}\left(s_{l-1}+\xi_{1} \mid 2 \tau\right) \theta_{4}\left(s_{l}+\xi_{2} \mid 2 \tau\right)\right)\left(d_{1} d_{2}-b_{1} a_{2}\right) \\
& +\left(\theta_{4}\left(t_{l-1}-u \mid 2 \tau\right) \theta_{4}\left(t_{l+1}-u \mid 2 \tau\right) \theta_{1}\left(s_{l-1}+\xi_{1} \mid 2 \tau\right) \theta_{1}\left(s_{l}+\xi_{2} \mid 2 \tau\right)\right. \\
& \left.+\theta_{1}\left(t_{l-1}-u \mid 2 \tau\right) \theta_{1}\left(t_{l+1}-u \mid 2 \tau\right) \theta_{4}\left(s_{l-1}+\xi_{1} \mid 2 \tau\right) \theta_{4}\left(s_{l}+\xi_{2} \mid 2 \tau\right)\right)\left(a_{1} c_{2}-c_{1} b_{2}\right) \\
& +\left(\theta_{4}\left(t_{l-1}-u \mid 2 \tau\right) \theta_{4}\left(t_{l+1}-u \mid 2 \tau\right) \theta_{4}\left(s_{l-1}+\xi_{1} \mid 2 \tau\right) \theta_{4}\left(s_{l}+\xi_{2} \mid 2 \tau\right)\right. \\
& \left.\left.+\theta_{1}\left(t_{l-1}-u \mid 2 \tau\right) \theta_{1}\left(t_{l+1}-u \mid 2 \tau\right) \theta_{1}\left(s_{l-1}+\xi_{1} \mid 2 \tau\right) \theta_{1}\left(s_{l}+\xi_{2} \mid 2 \tau\right)\right)\left(d_{1} a_{2}-b_{1} d_{2}\right)\right] \text {, } \\
& A_{2}^{(l)}=\frac{\gamma_{l-1} \gamma_{l+1}}{\mu(u ; s, t)}\left[\left(\theta_{4}\left(t_{l-1}-u \mid 2 \tau\right) \theta_{1}\left(t_{l+1}-u \mid 2 \tau\right) \theta_{1}\left(s_{l-1}+\xi_{1} \mid 2 \tau\right) \theta_{4}\left(s_{l}+\xi_{2} \mid 2 \tau\right)\right.\right. \\
& \left.-\theta_{1}\left(t_{l-1}-u \mid 2 \tau\right) \theta_{4}\left(t_{l+1}-u \mid 2 \tau\right) \theta_{4}\left(s_{l-1}+\xi_{1} \mid 2 \tau\right) \theta_{1}\left(s_{l}+\xi_{2} \mid 2 \tau\right)\right)\left(a_{1} b_{2}+c_{1} c_{2}\right) \\
& +\left(\theta_{4}\left(t_{l-1}-u \mid 2 \tau\right) \theta_{1}\left(t_{l+1}-u \mid 2 \tau\right) \theta_{4}\left(s_{l-1}+\xi_{1} \mid 2 \tau\right) \theta_{1}\left(s_{l}+\xi_{2} \mid 2 \tau\right)\right. \\
& \left.-\theta_{1}\left(t_{l-1}-u \mid 2 \tau\right) \theta_{4}\left(t_{l+1}-u \mid 2 \tau\right) \theta_{1}\left(s_{l-1}+\xi_{1} \mid 2 \tau\right) \theta_{4}\left(s_{l}+\xi_{2} \mid 2 \tau\right)\right)\left(d_{1} d_{2}+b_{1} a_{2}\right) \\
& +\left(\theta_{4}\left(t_{l-1}-u \mid 2 \tau\right) \theta_{4}\left(t_{l+1}-u \mid 2 \tau\right) \theta_{1}\left(s_{l-1}+\xi_{1} \mid 2 \tau\right) \theta_{1}\left(s_{l}+\xi_{2} \mid 2 \tau\right)\right. \\
& \left.-\theta_{1}\left(t_{l-1}-u \mid 2 \tau\right) \theta_{1}\left(t_{l+1}-u \mid 2 \tau\right) \theta_{4}\left(s_{l-1}+\xi_{1} \mid 2 \tau\right) \theta_{4}\left(s_{l}+\xi_{2} \mid 2 \tau\right)\right)\left(a_{1} c_{2}+c_{1} b_{2}\right) \\
& +\left(\theta_{4}\left(t_{l-1}-u \mid 2 \tau\right) \theta_{4}\left(t_{l+1}-u \mid 2 \tau\right) \theta_{4}\left(s_{l-1}+\xi_{1} \mid 2 \tau\right) \theta_{4}\left(s_{l}+\xi_{2} \mid 2 \tau\right)\right. \\
& \left.\left.-\theta_{1}\left(t_{l-1}-u \mid 2 \tau\right) \theta_{1}\left(t_{l+1}-u \mid 2 \tau\right) \theta_{1}\left(s_{l-1}+\xi_{1} \mid 2 \tau\right) \theta_{1}\left(s_{l}+\xi_{2} \mid 2 \tau\right)\right)\left(d_{1} a_{2}+b_{1} d_{2}\right)\right] \text {, } \\
& A_{3}^{(l)}=\frac{\gamma_{l-1} \gamma_{l+1}}{\mu(u ; s, t)}\left[\left(\theta_{4}\left(t_{l-1}-u \mid 2 \tau\right) \theta_{1}\left(t_{l+1}-u \mid 2 \tau\right) \theta_{1}\left(s_{l-1}+\xi_{1} \mid 2 \tau\right) \theta_{1}\left(s_{l}+\xi_{2} \mid 2 \tau\right)\right.\right. \\
& \left.+\theta_{1}\left(t_{l-1}-u \mid 2 \tau\right) \theta_{4}\left(t_{l+1}-u \mid 2 \tau\right) \theta_{4}\left(s_{l-1}+\xi_{1} \mid 2 \tau\right) \theta_{4}\left(s_{l}+\xi_{2} \mid 2 \tau\right)\right)\left(a_{1} a_{2}-c_{1} d_{2}\right) \\
& +\left(\theta_{4}\left(t_{l-1}-u \mid 2 \tau\right) \theta_{1}\left(t_{l+1}-u \mid 2 \tau\right) \theta_{4}\left(s_{l-1}+\xi_{1} \mid 2 \tau\right) \theta_{4}\left(s_{l}+\xi_{2} \mid 2 \tau\right)\right. \\
& \left.+\theta_{1}\left(t_{l-1}-u \mid 2 \tau\right) \theta_{4}\left(t_{l+1}-u \mid 2 \tau\right) \theta_{1}\left(s_{l-1}+\xi_{1} \mid 2 \tau\right) \theta_{1}\left(s_{l}+\xi_{2} \mid 2 \tau\right)\right)\left(d_{1} c_{2}-b_{1} b_{2}\right) \\
& +\left(\theta_{4}\left(t_{l-1}-u \mid 2 \tau\right) \theta_{4}\left(t_{l+1}-u \mid 2 \tau\right) \theta_{1}\left(s_{l-1}+\xi_{1} \mid 2 \tau\right) \theta_{4}\left(s_{l}+\xi_{2} \mid 2 \tau\right)\right. \\
& \left.+\theta_{1}\left(t_{l-1}-u \mid 2 \tau\right) \theta_{1}\left(t_{l+1}-u \mid 2 \tau\right) \theta_{4}\left(s_{l-1}+\xi_{1} \mid 2 \tau\right) \theta_{1}\left(s_{l}+\xi_{2} \mid 2 \tau\right)\right)\left(a_{1} d_{2}-c_{1} a_{2}\right) \\
& +\left(\theta_{4}\left(t_{l-1}-u \mid 2 \tau\right) \theta_{4}\left(t_{l+1}-u \mid 2 \tau\right) \theta_{4}\left(s_{l-1}+\xi_{1} \mid 2 \tau\right) \theta_{1}\left(s_{l}+\xi_{2} \mid 2 \tau\right)\right. \\
& \left.\left.+\theta_{1}\left(t_{l-1}-u \mid 2 \tau\right) \theta_{1}\left(t_{l+1}-u \mid 2 \tau\right) \theta_{1}\left(s_{l-1}+\xi_{1} \mid 2 \tau\right) \theta_{4}\left(s_{l}+\xi_{2} \mid 2 \tau\right)\right)\left(d_{1} b_{2}-b_{1} c_{2}\right)\right] \text {, }
\end{aligned}
$$




$$
\begin{aligned}
A_{4}^{(l)}= & \frac{\gamma_{l-1} \gamma_{l+1}}{\mu(u ; s, t)}\left[\left(\theta_{4}\left(t_{l-1}-u \mid 2 \tau\right) \theta_{1}\left(t_{l+1}-u \mid 2 \tau\right) \theta_{1}\left(s_{l-1}+\xi_{1} \mid 2 \tau\right) \theta_{1}\left(s_{l}+\xi_{2} \mid 2 \tau\right)\right.\right. \\
& \left.-\theta_{1}\left(t_{l-1}-u \mid 2 \tau\right) \theta_{4}\left(t_{l+1}-u \mid 2 \tau\right) \theta_{4}\left(s_{l-1}+\xi_{1} \mid 2 \tau\right) \theta_{4}\left(s_{l}+\xi_{2} \mid 2 \tau\right)\right)\left(a_{1} a_{2}+c_{1} d_{2}\right) \\
& +\left(\theta_{4}\left(t_{l-1}-u \mid 2 \tau\right) \theta_{1}\left(t_{l+1}-u \mid 2 \tau\right) \theta_{4}\left(s_{l-1}+\xi_{1} \mid 2 \tau\right) \theta_{4}\left(s_{l}+\xi_{2} \mid 2 \tau\right)\right. \\
& \left.-\theta_{1}\left(t_{l-1}-u \mid 2 \tau\right) \theta_{4}\left(t_{l+1}-u \mid 2 \tau\right) \theta_{1}\left(s_{l-1}+\xi_{1} \mid 2 \tau\right) \theta_{1}\left(s_{l}+\xi_{2} \mid 2 \tau\right)\right)\left(d_{1} c_{2}+b_{1} b_{2}\right) \\
& +\left(\theta_{4}\left(t_{l-1}-u \mid 2 \tau\right) \theta_{4}\left(t_{l+1}-u \mid 2 \tau\right) \theta_{1}\left(s_{l-1}+\xi_{1} \mid 2 \tau\right) \theta_{4}\left(s_{l}+\xi_{2} \mid 2 \tau\right)\right. \\
& \left.-\theta_{1}\left(t_{l-1}-u \mid 2 \tau\right) \theta_{1}\left(t_{l+1}-u \mid 2 \tau\right) \theta_{4}\left(s_{l-1}+\xi_{1} \mid 2 \tau\right) \theta_{1}\left(s_{l}+\xi_{2} \mid 2 \tau\right)\right)\left(a_{1} d_{2}+c_{1} a_{2}\right) \\
& +\left(\theta_{4}\left(t_{l-1}-u \mid 2 \tau\right) \theta_{4}\left(t_{l+1}-u \mid 2 \tau\right) \theta_{4}\left(s_{l-1}+\xi_{1} \mid 2 \tau\right) \theta_{1}\left(s_{l}+\xi_{2} \mid 2 \tau\right)\right. \\
& \left.\left.-\theta_{1}\left(t_{l-1}-u \mid 2 \tau\right) \theta_{1}\left(t_{l+1}-u \mid 2 \tau\right) \theta_{1}\left(s_{l-1}+\xi_{1} \mid 2 \tau\right) \theta_{4}\left(s_{l}+\xi_{2} \mid 2 \tau\right)\right)\left(d_{1} b_{2}+b_{1} c_{2}\right)\right]
\end{aligned}
$$

For rational $\eta=2 P / Q$ the vector $\left|\Psi^{l}(u)\right\rangle$ is $Q$-periodic in $l$. The (off-shell) Bethe vectors $\left|\Psi_{\nu}(u)\right\rangle(\nu=0,1, \ldots, Q-1)$ are defined as finite Fourier transforms of $\left|\Psi^{l}(u)\right\rangle$ :

$$
\left|\Psi_{\nu}(u)\right\rangle=\sum_{l=0}^{Q-1} e^{-2 \pi i P l \nu / Q}\left|\Psi^{l}(u)\right\rangle .
$$

This example illustrates that any direct calculations with the Bethe vectors, even in the simplest case $N=2$, are hardly possible.

The result for scalar products. Combining transformation properties under shifts of the variables with results of computer simulations, one can suggest the following formula for the scalar products at $N=2$ :

$$
\left\langle\Psi_{\nu}(v) \mid \Psi_{\mu}(u)\right\rangle=\phi_{1}^{(\nu \mu)}(v-u, x) \phi_{2}^{(\nu)}(v, y) \theta_{1}(u-v) T_{11}^{(\nu \mu)}(v-u) .
$$

The function $\phi_{1}^{(\nu \mu)}(r, x)$ is given by (5.49) for even $Q$ and by (5.50) for odd $Q$. The determinant of the matrix $T_{j k}^{(\nu \mu)}$ reduces to the element $T_{11}^{(\nu \mu)}$ (see (5.30)). Plugging $r=$ $v-u$ into (5.30) we get the $\nu$-independent expression

$$
T_{11}^{(\nu \mu)}=-\frac{\theta_{1}^{\prime}(0) \theta_{1}(\eta)}{\theta_{1}^{2}(v-u)}\left(a(u) e^{i \pi \mu \eta}-d(u) e^{-i \pi \mu \eta}\right) .
$$

The function $\phi_{2}^{(\nu)}(v, y)$ is

$$
\phi_{2}^{(\nu)}(v, y)=C(\eta, \tau) \chi^{(\nu)}(v) d(v) e^{2 \pi i \nu y} \theta_{1}^{2}(y+v),
$$

where

$$
\chi^{(\nu)}(v)=\left.e^{2 \pi i \nu(v-\eta)} \theta_{1}\left(v-\xi_{1}\right) \theta_{1}\left(v-\xi_{2}\right) \partial_{z} \log \frac{a(z)}{d(z)}\right|_{z=v}
$$

and

$$
C(\eta, \tau)= \begin{cases}\frac{2 Q^{2} \theta_{1}^{\prime}(0 \mid Q \tau / 2)}{\left(\theta_{1}^{\prime}(0)\right)^{3} \theta_{2}^{2}(0) \theta_{1}(\eta)} & \text { for even } Q \\ \frac{2 Q^{2} \theta_{1}^{\prime}(0 \mid Q \tau)}{\left(\theta_{1}^{\prime}(0)\right)^{3} \theta_{2}^{2}(0) \theta_{1}(\eta)} & \text { for odd } Q\end{cases}
$$




\section{Free fermions}

The case $\eta=1 / 2(Q=4)$ corresponds to free fermions. The Bethe equations drastically simplify in this case; they have the form

$$
e^{\pi i \nu} \frac{a\left(v_{j}\right)}{d\left(v_{j}\right)}=(-1)^{n-1} .
$$

For the case of free fermions it is possible to obtain more explicit expressions for the scalar products. The matrix $T_{j k}^{(\nu \mu)}(r)$ is given by

$$
\begin{aligned}
T_{j k}^{(\nu \mu)}(r)= & \frac{\theta_{1}^{\prime}(0)}{\theta_{1}(r)}\left(\prod_{p=1}^{n} \frac{\theta_{2}\left(u_{k}-v_{p}\right)}{\theta_{1}\left(u_{k}-v_{p}\right)}\right) \\
& \times\left[(-1)^{n} a\left(u_{k}\right)\left(e^{i \pi \nu / 2} \frac{\theta_{1}\left(u_{k}-v_{j}+r\right)}{\theta_{1}\left(u_{k}-v_{j}\right)}-e^{i \pi \mu / 2} \frac{\theta_{2}\left(u_{k}-v_{j}+r\right)}{\theta_{2}\left(u_{k}-v_{j}\right)}\right)\right. \\
& \left.+d\left(u_{k}\right)\left(e^{-i \pi \nu / 2} \frac{\theta_{1}\left(u_{k}-v_{j}+r\right)}{\theta_{1}\left(u_{k}-v_{j}\right)}-e^{-i \pi \mu / 2} \frac{\theta_{2}\left(u_{k}-v_{j}+r\right)}{\theta_{2}\left(u_{k}-v_{j}\right)}\right)\right] .
\end{aligned}
$$

Case $\boldsymbol{\mu}=\boldsymbol{\nu} . \quad$ Set $\mu=\nu$. Then

$$
\begin{aligned}
T_{j k}^{(\nu \nu)}(r)= & \frac{\theta_{1}^{\prime}(0)}{\theta_{1}(r)}\left(\prod_{p=1}^{n} \frac{\theta_{2}\left(u_{k}-v_{p}\right)}{\theta_{1}\left(u_{k}-v_{p}\right)}\right) \\
& \times\left((-1)^{n} e^{i \pi \nu / 2} a\left(u_{k}\right)+e^{-i \pi \nu / 2} d\left(u_{k}\right)\right) H^{(-)}\left(u_{k}, v_{j}\right),
\end{aligned}
$$

where

$$
\begin{aligned}
H^{(-)}\left(u_{k}, v_{j}\right) & =\frac{\theta_{1}\left(u_{k}-v_{j}+r\right)}{\theta_{1}\left(u_{k}-v_{j}\right)}-\frac{\theta_{2}\left(u_{k}-v_{j}+r\right)}{\theta_{2}\left(u_{k}-v_{j}\right)} \\
& =\frac{2 \theta_{1}(r \mid 2 \tau)}{\theta_{4}(0 \mid 2 \tau)} \frac{\theta_{4}\left(2 u_{k}-2 v_{j}+r \mid 2 \tau\right)}{\theta_{1}\left(2 u_{k}-2 v_{j} \mid 2 \tau\right)}
\end{aligned}
$$

and we arrive at

$$
\begin{aligned}
& T_{j k}^{(\nu \nu)}(r)=\frac{2 \theta_{1}(r \mid 2 \tau) \theta_{1}^{\prime}(0 \mid \tau)}{\theta_{1}(r \mid \tau) \theta_{4}(0 \mid 2 \tau)}\left(\prod_{p=1}^{n} \frac{\theta_{2}\left(u_{k}-v_{p}\right)}{\theta_{1}\left(u_{k}-v_{p}\right)}\right) \\
& \quad \times\left((-1)^{n} e^{i \pi \nu / 2} a\left(u_{k}\right)+e^{-i \pi \nu / 2} d\left(u_{k}\right)\right) \frac{\theta_{4}\left(2 u_{k}-2 v_{j}+r \mid 2 \tau\right)}{\theta_{1}\left(2 u_{k}-2 v_{j} \mid 2 \tau\right)},
\end{aligned}
$$

i.e. it is the elliptic Cauchy matrix (multiplied by a diagonal matrix).

Case $\boldsymbol{\mu} \neq \boldsymbol{\nu}$. If $\mu \neq \nu$, then generically we cannot obtain the Cauchy matrix. However, if $\mu=\nu+2$, then $e^{i \pi \mu / 2}=-e^{i \pi \nu / 2}$, and the matrix elements $T_{j k}^{(\nu, \nu+2)}(r)$ take the form

$$
T_{j k}^{(\nu, \nu+2)}(r)=\frac{\theta_{1}^{\prime}(0)}{\theta_{1}(r)}\left(\prod_{p=1}^{n} \frac{\theta_{2}\left(u_{k}-v_{p}\right)}{\theta_{1}\left(u_{k}-v_{p}\right)}\right)\left((-1)^{n} e^{i \pi \nu / 2} a\left(u_{k}\right)-e^{-i \pi \nu / 2} d\left(u_{k}\right)\right) H^{(+)}\left(u_{k}, v_{j}\right),
$$


where

$$
\begin{aligned}
H^{(+)}\left(u_{k}, v_{j}\right) & =\frac{\theta_{1}\left(u_{k}-v_{j}+r\right)}{\theta_{1}\left(u_{k}-v_{j}\right)}+\frac{\theta_{2}\left(u_{k}-v_{j}+r\right)}{\theta_{2}\left(u_{k}-v_{j}\right)} \\
& =\frac{2 \theta_{4}(r \mid 2 \tau)}{\theta_{4}(0 \mid 2 \tau)} \frac{\theta_{1}\left(2 u_{k}-2 v_{j}+r \mid 2 \tau\right)}{\theta_{1}\left(2 u_{k}-2 v_{j} \mid 2 \tau\right)},
\end{aligned}
$$

and finally we arrive at

$$
\begin{aligned}
T_{j k}^{(\nu, \nu+2)}(r)= & \frac{2 \theta_{4}(r \mid 2 \tau) \theta_{1}^{\prime}(0 \mid \tau)}{\theta_{1}(r \mid \tau) \theta_{4}(0 \mid 2 \tau)}\left(\prod_{p=1}^{n} \frac{\theta_{2}\left(u_{k}-v_{p}\right)}{\theta_{1}\left(u_{k}-v_{p}\right)}\right) \\
& \times\left((-1)^{n} e^{i \pi \nu / 2} a\left(u_{k}\right)-e^{-i \pi \nu / 2} d\left(u_{k}\right)\right) \frac{\theta_{1}\left(2 u_{k}-2 v_{j}+r \mid 2 \tau\right)}{\theta_{1}\left(2 u_{k}-2 v_{j} \mid 2 \tau\right)}
\end{aligned}
$$

Elliptic Cauchy determinants. Below we use the notation

$$
W_{n}(\bar{u}, \bar{v} \mid \tau)=\frac{\prod_{1 \leq a<b \leq n} \theta_{1}\left(u_{a}-u_{b} \mid \tau\right) \theta_{1}\left(v_{b}-v_{a} \mid \tau\right)}{\prod_{1 \leq a, b \leq n} \theta_{1}\left(u_{a}-v_{b} \mid \tau\right)} .
$$

Then the explicit formula for the elliptic Cauchy determinant tells us that

$$
\operatorname{det}_{1 \leq j, k \leq n}\left(\frac{\theta_{1}\left(2 u_{k}-2 v_{j}+r \mid 2 \tau\right)}{\theta_{1}\left(2 u_{k}-2 v_{j} \mid 2 \tau\right)}\right)=\theta_{1}^{n-1}(r \mid 2 \tau) \theta_{1}(r+2 U-2 V \mid 2 \tau) W_{n}(2 \bar{u}, 2 \bar{v} \mid 2 \tau),
$$

and

$$
\operatorname{det}_{1 \leq j, k \leq n}\left(\frac{\theta_{4}\left(2 u_{k}-2 v_{j}+r \mid 2 \tau\right)}{\theta_{1}\left(2 u_{k}-2 v_{j} \mid 2 \tau\right)}\right)=\theta_{4}^{n-1}(r \mid 2 \tau) \theta_{4}(r+2 U-2 V \mid 2 \tau) W_{n}(2 \bar{u}, 2 \bar{v} \mid 2 \tau) .
$$

Here

$$
U=\sum_{k=1}^{n} u_{k}, \quad V=\sum_{k=1}^{n} v_{k} .
$$

Equation (C.10) follows from (C.9) if we use the relation

$$
\theta_{4}(x \mid 2 \tau)=-i e^{i \pi(x+\tau / 2)} \theta_{1}(x+\tau \mid 2 \tau) .
$$

Determinant of the matrix $T_{j k}^{(\nu \mu)}$.

- Case $\mu=\nu$. The above results yield

$$
\begin{aligned}
\operatorname{det} T_{j k}^{(\nu \nu)}(r)= & \left(\frac{2 \theta_{1}(r \mid 2 \tau) \theta_{4}(r \mid 2 \tau) \theta_{1}^{\prime}(0 \mid \tau)}{\theta_{1}(r \mid \tau) \theta_{4}(0 \mid 2 \tau)}\right)^{n}\left(\prod_{a, b=1}^{n} \frac{\theta_{2}\left(u_{a}-v_{b}\right)}{\theta_{1}\left(u_{a}-v_{b}\right)}\right) \\
& \times \prod_{p=1}^{n}\left((-1)^{n} e^{i \pi \nu / 2} a\left(u_{p}\right)+e^{-i \pi \nu / 2} d\left(u_{p}\right)\right) \\
& \times \frac{\theta_{4}(r+2 U-2 V \mid 2 \tau)}{\theta_{4}(r \mid 2 \tau)} W_{n}(2 \bar{u}, 2 \bar{v} \mid 2 \tau) .
\end{aligned}
$$


This expression can be simplified using the identity $\theta_{1}(x \mid \tau) \theta_{2}(0 \mid \tau)=$ $2 \theta_{1}(x \mid 2 \tau) \theta_{4}(x \mid 2 \tau)$ and its derivative at $x=0$ :

$$
\begin{aligned}
\operatorname{det} T_{j k}^{(\nu \nu)}(r)= & \left(2 \theta_{1}^{\prime}(0 \mid 2 \tau)\right)^{n} \frac{\theta_{4}(r+2 U-2 V \mid 2 \tau)}{\theta_{4}(r \mid 2 \tau)}\left(\prod_{a, b=1}^{n} \frac{\theta_{2}\left(u_{a}-v_{b}\right)}{\theta_{1}\left(u_{a}-v_{b}\right)}\right) \\
& \times W_{n}(2 \bar{u}, 2 \bar{v} \mid 2 \tau) \prod_{p=1}^{n}\left((-1)^{n} e^{i \pi \nu / 2} a\left(u_{p}\right)+e^{-i \pi \nu / 2} d\left(u_{p}\right)\right) .
\end{aligned}
$$

Observe that in the case of the scalar product we should set $r=V-U$, and then the ratio of the $\theta_{4}$-functions disappears:

$$
\frac{\theta_{4}(r+2 U-2 V \mid 2 \tau)}{\theta_{4}(r \mid 2 \tau)}=\frac{\theta_{4}(-r \mid 2 \tau)}{\theta_{4}(r \mid 2 \tau)}=1 .
$$

- Case $\mu \neq \nu$. In a similar way, we obtain for $\mu=\nu+2$ :

$$
\begin{aligned}
\operatorname{det} T_{j k}^{(\nu, \nu+2)}(r)= & \left(2 \theta_{1}^{\prime}(0 \mid 2 \tau)\right)^{n} \frac{\theta_{1}(r+2 U-2 V \mid 2 \tau)}{\theta_{1}(r \mid 2 \tau)}\left(\prod_{a, b=1}^{n} \frac{\theta_{2}\left(u_{a}-v_{b}\right)}{\theta_{1}\left(u_{a}-v_{b}\right)}\right) \\
& \times W_{n}(2 \bar{u}, 2 \bar{v} \mid 2 \tau) \prod_{p=1}^{n}\left((-1)^{n} e^{i \pi \nu / 2} a\left(u_{p}\right)-e^{-i \pi \nu / 2} d\left(u_{p}\right)\right) .
\end{aligned}
$$

Again, in the case of the scalar product the ratio of the $\theta_{1}$-functions simplifies, but now it gives the minus sign:

$$
\frac{\theta_{1}(r+2 U-2 V \mid 2 \tau)}{\theta_{1}(r \mid 2 \tau)}=\frac{\theta_{1}(-r \mid 2 \tau)}{\theta_{1}(r \mid 2 \tau)}=-1
$$

In the case when $\mu-\nu$ is odd no simple result for $\operatorname{det} T_{j k}^{(\nu \mu)}(r)$ is available. However, in this case the scalar product vanishes due to the selection rule: $\left\langle\Psi_{\nu}(\bar{v}) \mid \Psi_{\mu}(\bar{u})\right\rangle=0$ if $\mu-\nu$ is odd (see section 5.7).

Scalar products. Summarizing the above results, we obtain an explicit representation for the scalar product

$$
X_{\mu \nu}^{(n)}(\bar{v}, \bar{u})=\left\langle\Psi_{\nu}(\bar{v}) \mid \Psi_{\mu}(\bar{u})\right\rangle
$$

The result is

$$
\begin{aligned}
X_{\mu \nu}^{(n)}(\bar{v}, \bar{u})= & \pm\left(2 \theta_{1}^{\prime}(0 \mid 2 \tau)\right)^{n} \phi_{1}^{(\nu \mu)}(r, x) \phi_{2}^{(\nu)}(\bar{v}, y)\left(\prod_{a, b=1}^{n} \frac{\theta_{2}\left(u_{a}-v_{b}\right)}{\theta_{1}\left(u_{a}-v_{b}\right)}\right) \frac{W_{n}(2 \bar{u}, 2 \bar{v} \mid 2 \tau)}{W_{n}(\bar{u}, \bar{v} \mid \tau)} \\
& \times \prod_{p=1}^{n}\left((-1)^{n} e^{i \pi \nu / 2} a\left(u_{p}\right) \pm e^{-i \pi \nu / 2} d\left(u_{p}\right)\right) .
\end{aligned}
$$

Here the upper sign corresponds to the case $\mu=\nu$ while the lower sign corresponds to the case $\mu=\nu+2$. The function $\phi_{1}^{(\nu \mu)}(r, x)$ is

$$
\phi_{1}^{(\nu \mu)}(r, x)=\delta_{\mu, \nu(\bmod 2)} e^{\pi i(\mu-\nu) x} \frac{\theta_{1}(r \mid \tau) \theta_{1}(r+2 x+(\mu-\nu) \tau / 2 \mid 2 \tau)}{\theta_{1}(2 x \mid 2 \tau) \theta_{1}(r+(\mu-\nu) \tau / 2 \mid 2 \tau)}
$$

(see (5.49)) and we recall that $r=\sum_{j} v_{j}-\sum_{j} u_{j}$. The function $\phi_{2}^{(\nu)}(\bar{v}, y)$ is unknown. 
For $\nu=\mu=0$ a complete analog of (C.14) exists in the $X X Z$ case. Actually, one can take the limit $\tau \rightarrow+i \infty$ in (C.14) and reproduce the $X X Z$ result up to a common factor. Respectively, in the case $\mu=\nu+2$, we reproduce the $X X Z$ scalar product of the twisted on-shell and off-shell vector. It is not clear how this twist appears.

The squared norm of the on-shell Bethe vector is

$$
\begin{aligned}
\left\langle\Psi_{\nu}(\bar{v}) \mid \Psi_{\nu}(\bar{v})\right\rangle= & (-1)^{n} \theta_{2}^{n}(0 \mid \tau) \frac{\theta_{1}^{\prime}(0 \mid \tau)}{\theta_{1}^{\prime}(0 \mid 2 \tau)} \phi_{2}^{(\nu)}(\bar{v}, y) e^{-\pi i \nu n / 2} \prod_{a \neq b}^{n} \frac{\theta_{2}\left(v_{a}-v_{b} \mid \tau\right)}{\theta_{1}\left(v_{a}-v_{b} \mid \tau\right)} \\
& \times \prod_{p=1}^{n}\left(\left.d\left(v_{p}\right) \partial_{v} \log \frac{a(v)}{d(v)}\right|_{v=v_{p}}\right) .
\end{aligned}
$$

The result for the specially normalized scalar product is

$$
\begin{aligned}
\frac{\left\langle\Psi_{\nu}(\bar{v}) \mid \Psi_{\mu}(\bar{u})\right\rangle}{\left\langle\Psi_{\nu}(\bar{v}) \mid \Psi_{\nu}(\bar{v})\right\rangle}= & \pm(-1)^{n} \frac{\theta_{1}^{\prime}(0 \mid 2 \tau)}{\theta_{1}^{\prime}(0 \mid \tau)}\left(\frac{2 \theta_{1}^{\prime}(0 \mid 2 \tau)}{\theta_{2}(0 \mid \tau)}\right)^{n} \frac{W_{n}(2 \bar{u}, 2 \bar{v} \mid 2 \tau)}{W_{n}(\bar{u}, \bar{v} \mid \tau)} \phi_{1}^{(\nu \mu)}(r, x) \\
& \times \prod_{a, b}^{n} \frac{\theta_{2}\left(u_{a}-v_{b} \mid \tau\right)}{\theta_{1}\left(u_{a}-v_{b} \mid \tau\right)} \prod_{a^{\prime} \neq b^{\prime}}^{n} \frac{\theta_{1}\left(v_{a^{\prime}}-v_{b^{\prime}} \mid \tau\right)}{\theta_{2}\left(v_{a^{\prime}}-v_{b^{\prime}} \mid \tau\right)} \\
& \times \prod_{p=1}^{n} \frac{(-1)^{n} e^{\pi i \nu} a\left(u_{p}\right) \pm d\left(u_{p}\right)}{\left.d\left(v_{p}\right) \partial_{v} \log (a(v) / d(v))\right|_{v=v_{p}}}
\end{aligned}
$$

\section{List of notations}

Here we list some notations for convenience as they appear in the text:

$\tau$ - modular parameter of elliptic functions;

$\eta$ - anisotropy parameter (or Planck constant) in the $R$-matrix;

$P, Q \in \mathbb{Z}_{+}$- coprime numbers entering $\eta=2 P / Q$;

$u$ - spectral parameter in of the $R$-matrix;

$W_{a}(u), a=0, \ldots, 3 ; a^{8 \mathrm{v}}(u), b^{8 \mathrm{v}}(u), c^{8 \mathrm{v}}(u), d^{8 \mathrm{v}}(u)$ - matrix elements of $R$-matrix (2.5);

$N$ - number of sites of the lattice row (an even integer);

$n=N / 2-$ number of Bethe roots, see also (3.61);

$\mathrm{a}(u), \mathrm{b}(u), \mathrm{c}(u), \mathrm{d}(u)$ - elementsof the $L$-operator (2.11);

$\xi_{1}, \ldots, \xi_{N}$ - inhomogeneity parameters, (2.13), (2.17);

$A(u), B(u), C(u), D(u)$ - operator matrix elements of the quantum monodromy matrix (2.14);

$c(u)=N(2 u+\eta+\tau)-2 \sum_{k=1}^{N} \xi_{k},(2.15) ;$

$\mathrm{U}_{a}=\left(\sigma_{a}\right)^{\otimes N},(2.18) ;$

$s, t$ - the parameters of intertwining (co)vectors $|\phi(s)\rangle(2.21)$; 
$\mathrm{a}_{k}^{\prime}(u), \mathrm{b}_{k}^{\prime}(u), \mathrm{c}_{k}^{\prime}(u), \mathrm{d}_{k}^{\prime}(u)$ - elements of the gauged transformed $L$-operator (2.37);

$s_{k}=s+k \eta, t_{k}=t+k \eta, \tau_{k}=\left(s_{k}+t_{k}\right) / 2$;

$\gamma_{k}$ - functions entering the (gauge transformation) matrix $M_{k}(u)(2.38)-(2.39)$;

$\left|\omega_{k}^{l}\right\rangle$ - local vacuum vectors (2.42);

$\left|\Omega^{l}\right\rangle=\left|\omega_{1}^{l}\right\rangle \otimes\left|\omega_{2}^{l}\right\rangle \otimes \ldots \otimes\left|\omega_{N}^{l}\right\rangle$ - global vacuum vectors $(2.45) ;$

$A_{k, l}(u), B_{k, l}(u), C_{k, l}(u), D_{k, l}(u)$ - elements of $\mathcal{T}_{k, l}(u)(3.1)-(3.2)$;

$\left|\Psi^{l}\left(u_{1}, \ldots, u_{n}\right)\right\rangle$ - vectors $(3.16)$;

$\Phi(u, v)$ - (Kronecker) function (3.20);

$\left|\Psi_{\nu}\left(u_{1}, \ldots, u_{n}\right)\right\rangle$ - right Bethe vector, Fourier transform of $\left|\Psi^{l}\right\rangle$ (3.21);

$\bar{A}_{k, l}(u), \bar{B}_{k, l}(u), \bar{C}_{k, l}(u), \bar{D}_{k, l}(u)$ - operators (3.28);

$\left\langle\Psi^{l}\left(v_{1}, \ldots, v_{n}\right)\right|$ - left vector (3.30);

$\left\langle\Psi_{\nu}\left(v_{1}, \ldots, v_{n}\right)\right|$ — left Bethe vector, Fourier transform of $\left\langle\Psi^{l}\right|(3.33)$;

$\sigma\left(v_{1}, \ldots, v_{n}\right)=\sum_{i=1}^{n} v_{i}-\frac{1}{2} \sum_{k=1}^{N} \xi_{k}+\frac{1}{2} n \eta$, the function (3.54);

$x=(s+t+1) / 2, y=(s-t) / 2-$ variables $(3.62) ;$

$\nu_{a}=0,1-$ the numbers from (3.63);

$\nu=-n-\nu_{3}$

$a(u)=\prod_{i=1}^{N} \theta_{1}\left(u-\xi_{i}+\eta\right), d(u)=\prod_{i=1}^{N} \theta_{1}\left(u-\xi_{i}\right)$ - the functions (4.5);

Bethe equations - (3.25), (4.22);

sum rule - (4.24);

$v_{1}, \ldots, v_{n}-$ Bethe roots, solutions of (4.22) and (4.24);

$g(u, v), f(u, v), h(u, v)$ - functions (5.1)-(5.3);

$X_{j}^{l}$ — the scalar products $\left\langle\Psi_{\nu}(\bar{v}) \mid \Psi^{l}\left(\bar{u}_{j}\right)\right\rangle(5.5)$;

$T_{\nu}(u)$ - function (3.23), (5.7);

$w=w_{n+1}=l \eta+\check{U}+w_{0},(5.22) ;$

$\check{U}=\sum_{j=1}^{n+1} u_{j}$

$r=\sum_{p=1}^{n} v_{p}-\frac{1}{2}(s+t+1)+w_{0},(5.24) ;$

$Y_{k}^{(\mu)}$ — weighted Fourier transform of $X_{j}^{l}(5.26)$;

$U_{n+1}=U=\sum_{p=1}^{n} u_{p}, V=\sum_{p=1}^{n} v_{p},(5.27) ;$ 
$T_{i k}^{(\nu \mu)}(r)$ - matrix (5.29)-(5.30);

$W_{n}$ - function (5.34), (5.55);

$\phi^{(\nu, \mu)}(\bar{v}, r)$ - function (5.36), (5.47);

$\phi_{1}^{(\nu, \mu)}(r, x), \phi_{2}^{(\nu)}(\bar{v}, y)$ - functions $(5.47),(5.49)-(5.51),(5.52)$;

$S_{\nu \mu}(\bar{v}, \bar{u})$ - specially normalized scalar products (5.65).

Open Access. This article is distributed under the terms of the Creative Commons Attribution License (CC-BY 4.0), which permits any use, distribution and reproduction in any medium, provided the original author(s) and source are credited.

\section{References}

[1] B. Sutherland, Two-dimensional hydrogen bonded crystals without the ice rule, J. Math. Phys. 11 (1970) 3183.

[2] C. Fan and F.Y. Wu, General lattice model of phase transitions, Phys. Rev. B 2 (1970) 723 [INSPIRE].

[3] R.J. Baxter, Eight-vertex model in lattice statistics, Phys. Rev. Lett. 26 (1971) 832 [INSPIRE].

[4] R. Baxter, Exactly solved models in statistical mechanics, Academic Press, U.S.A. (1982).

[5] W. Heisenberg, Zur Theorie des Ferromagnetismus, Z. Phys. 49 (1928) 619 [InSPIRE].

[6] R.J. Baxter, One-dimensional anisotropic Heisenberg chain, Phys. Rev. Lett. 26 (1971) 834 [INSPIRE].

[7] R. Baxter, Partition function of the eight-vertex lattice model, Ann. Phys. 70 (1972) 193.

[8] R. Baxter, Eight-vertex model in lattice statistics and one-dimensional anisotropic Heisenberg chain. I. Some fundamental eigenvectors, Ann. Phys. 76 (1973) 1.

[9] R. Baxter, Eight-vertex model in lattice statistics and one-dimensional anisotropic Heisenberg chain. II. Equivalence to a Generalized Ice-type Lattice Model, Ann. Phys. 76 (1973) 25.

[10] R. Baxter, Eight-vertex model in lattice statistics and one-dimensional anisotropic Heisenberg chain. III. Eigenvectors of the transfer matrix and Hamiltonian, Ann. Phys. 76 (1973) 48.

[11] A. Klümper and J. Zittartz, Eigenvalues of the eight-vertex model transfer matrix and the spectrum of the XYZ Hamiltonian, Z. Phys. B 71 (1988) 495.

[12] A. Klümper and J. Zittartz, The eight-vertex model: spectrum of the transfer matrix and classification of the excited states, Z. Phys. B 75 (1989) 371.

[13] T. Takebe, Bethe Ansatz for higher spin eight vertex models, q-alg/9504027.

[14] K. Fabricius and B. McCoy, New developments in the eight vertex model, J. Stat. Phys. 111 (2003) 323 [cond-mat/0207177].

[15] K. Fabricius and B.M. McCoy, New developments in the eight vertex model II. Chains of odd length, J. Stat. Phys. 120 (2005) 37 [cond-mat/0410113].

[16] K. Fabricius and B. McCoy, The eight vertex model. New results, cond-mat/0612699.

[17] K. Fabricius and B.M. McCoy, The TQ equation of the 8 vertex model for complex elliptic roots of unity, J. Phys. 40 (2007) 14893 [arXiv:0709.0903] [INSPIRE]. 
[18] K. Fabricius, A new Q-matrix in the eight-vertex model, J. Phys. 40 (2007) 4075 [cond-mat/0610481] [INSPIRE].

[19] V.V. Bazhanov and V.V. Mangazeev, Analytic theory of the eight-vertex model, Nucl. Phys. B 775 (2007) 225 [hep-th/0609153] [INSPIRE].

[20] T. Takebe, $Q$-operators for higher spin eight vertex models with an even number of sites, Lett. Math. Phys. 106 (2016) 319 [arXiv:1509.08616] [INSPIRE].

[21] E.K. Sklyanin, On some algebraic structures associated with the Yang-Baxter equation, Funct. Anal. Appl. 16 (1982) 263.

[22] O. Foda et al., An elliptic quantum algebra for sl(2), Lett. Math. Phys. 32 (1994) 259 [hep-th/9403094] [INSPIRE].

[23] C. Fronsdal, Quasi-Hopf deformations of quantum groups, Lett. Math. Phys. 40 (1997) 117 [q-alg/9611028] [INSPIRE].

[24] M. Jimbo, H. Konno, S. Odake and J. Shiraishi, Quasi-Hopf twistors for elliptic quantum groups, Transform. Groups 4 (1999) 303 [q-alg/9712029].

[25] E. Buffenoir, Ph. Roche and V. Terras, Universal vertex-IRF transformation for quantum affine algebras, J. Math. Phys. 53 (2012) 103515 [arXiv:0707.0955].

[26] J.D. Johnson, S. Krinsky and B.M. McCoy, Vertical-arrow correlation length in the eight-vertex model and the low-lying excitations of the X-Y-Z Hamiltonian, Phys. Rev. A 8 (1973) 2526 [INSPIRE].

[27] M. Jimbo, T. Miwa and A. Nakayashiki, Difference equations for the correlation functions of the eight vertex model, J. Phys. 26 (1993) 2199 [hep-th/9211066] [INSPIRE].

[28] M. Lashkevich and Y. Pugai, Free field construction for correlation functions of the eight vertex model, Nucl. Phys. B 516 (1998) 623 [hep-th/9710099] [INSPIRE].

[29] M. Lashkevich, Free field construction for the eight vertex model: representation for form-factors, Nucl. Phys. B 621 (2002) 587 [hep-th/0103144] [INSPIRE].

[30] J. Shiraishi, Free field constructions for the elliptic algebra $\mathcal{A}_{q, p}\left(\widehat{s l}_{2}\right)$ and Baxter's eight-vertex model, Int. J. Mod. Phys. A 19 (2004) 363 [math.QA/0302097].

[31] H. Boos, M. Jimbo, T. Miwa, F. Smirnov and Y. Takeyama, Traces on the Sklyanin algebra and correlation functions of the eight-vertex model, J. Phys. 38 (2005) 7629 [hep-th/0504072] [INSPIRE].

[32] L.D. Faddeev, E.K. Sklyanin and L.A. Takhtajan, The quantum inverse problem method. 1, Theor. Math. Phys. 40 (1980) 688 [inSPIRE].

[33] L.D. Faddeev, How algebraic Bethe ansatz works for integrable model, in Quantum symmetries, A. Connes et al. eds., North Holland, The Netherlands (1998), hep-th/9605187 [INSPIRE].

[34] L. Takhtajan and L. Faddeev, The quantum method of the inverse problem and the Heisenberg XYZ model, Usp. Mat. Nauk 34 (1979) 13 [Russ. Math. Surveys 34 (1979) 11].

[35] A.G. Izergin and V.E. Korepin, The quantum inverse scattering method approach to correlation functions, Commun. Math. Phys. 94 (1981) 67.

[36] N. Kitanine, J.M. Maillet and V. Terras, Form factors of the XXZ Heisenberg spin- $\frac{1}{2}$ finite chain, Nucl. Phys. B 554 (1999) 647 [math-ph/9807020] [INSPIRE]. 
[37] F. Gohmann and V.E. Korepin, Solution of the quantum inverse problem, J. Phys. 33 (2000) 1199 [hep-th/9910253] [INSPIRE].

[38] J.M. Maillet and V. Terras, On the quantum inverse scattering problem, Nucl. Phys. B $\mathbf{5 7 5}$ (2000) 627 [hep-th/9911030] [INSPIRE].

[39] M. Gaudin, Modèles exacts en mécanique statistique: la méthode de Bethe et ses généralisations, Centre d'Etudes Nucléaires de Saclay, CEA-N-1559:1 (1972).

[40] M. Gaudin, La fonction d'onde de Bethe, Masson, France (1983).

[41] M. Gaudin, B.M. McCoy and T.T. Wu, Normalization sum for the Bethe's hypothesis wave functions of the Heisenberg-Ising chain, Phys. Rev. D 23 (1981) 417 [INSPIRE].

[42] V.E. Korepin, Calculation of norms of Bethe wave functions, Commun. Math. Phys. 86 (1982) 391.

[43] N.A. Slavnov, Calculation of scalar products of wave functions and form factors in the framework of the algebraic Bethe Ansatz, Theor. Math. Phys. 79 (1989) 502.

[44] Y.-S. Wang, The scalar products and the norm of Bethe eigenstates for the boundary XXX Heisenberg spin-1/2 finite chain, Nucl. Phys. B 622 (2002) 633 [InSPIRE].

[45] N. Kitanine et al., Correlation functions of the open XXZ chain I, J. Stat. Mech. 0710 (2007) P10009 [arXiv:0707.1995] [InSPIRE].

[46] S. Belliard and R.A. Pimenta, Slavnov and Gaudin-Korepin formulas for models without U(1) symmetry: the twisted $X X X$ chain, SIGMA 11 (2015) 099 [arXiv: 1506. 06550] [INSPIRE].

[47] S. Belliard and R.A. Pimenta, Slavnov and Gaudin-Korepin formulas for models without $U$ (1) symmetry: the XXX chain on the segment, J. Phys. 49 (2016) 17LT01 [arXiv: 1507.03242] [INSPIRE].

[48] D. Levy-Bencheton and V. Terras, Algebraic Bethe Ansatz approach to form factors and correlation functions of the cyclic eight-vertex solid-on-solid model, J. Stat. Mech. Theory Exp. 04 (2013) P04015 [arXiv: 1212.0246].

[49] S. Belliard and N.A. Slavnov, Why scalar products in the algebraic Bethe ansatz have determinant representation, JHEP 10 (2019) 103 [arXiv: 1908.00032] [INSPIRE].

[50] V.E. Korepin, Dual field formulation of quantum integrable models, Commun. Math. Phys. 113 (1987) 177.

[51] T. Kojima, V.E. Korepin and N.A. Slavnov, Determinant representation for dynamical correlation functions of the quantum nonlinear Schrödinger equation, Commun. Math. Phys. 188 (1997) 657 [hep-th/9611216] [INSPIRE].

[52] V.E. Korepin, N.M. Bogoliubov and A.G. Izergin, Quantum inverse scattering method and correlation functions, Cambridge University Press, Cambridge U.K. (1993).

[53] M. Jimbo, K. Miki, T. Miwa and A. Nakayashiki, Correlation functions of the XXZ model for $\Delta<-1$, Phys. Lett. A 168 (1992) 256 [hep-th/9205055] [INSPIRE].

[54] N. Kitanine, J.M. Maillet and V. Terras, Correlation functions of the XXZ Heisenberg spin- $\frac{1}{2}$ chain in a magnetic field, Nucl. Phys. B 567 (2000) 554 [math-ph/9907019] [INSPIRE].

[55] F. Gohmann, A. Klumper and A. Seel, Integral representations for correlation functions of the XXZ chain at finite temperature, J. Phys. 37 (2004) 7625 [hep-th/0405089] [INSPIRE]. 
[56] N. Kitanine, J.M. Maillet, N.A. Slavnov and V. Terras, Master equation for spin-spin correlation functions of the XXZ chain, Nucl. Phys. B 712 (2005) 600 [hep-th/0406190] [INSPIRE].

[57] N. Kitanine et al., Algebraic Bethe ansatz approach to the asymptotic behavior of correlation functions, J. Stat. Mech. 0904 (2009) P04003 [arXiv:0808.0227] [INSPIRE].

[58] N. Kitanine et al., Form factor approach to the asymptotic behavior of correlation functions in critical models, J. Stat. Mech. 1112 (2011) P12010 [arXiv:1110.0803] [INSPIRE].

[59] N. Kitanine et al., Form factor approach to dynamical correlation functions in critical models, J. Stat. Mech. 1209 (2012) P09001 [arXiv:1206.2630] [INSPIRE].

[60] J.-S. Caux and J.-M. Maillet, Computation of dynamical correlation functions of Heisenberg chains in a field, Phys. Rev. Lett. 95 (2005) 077201 [cond-mat/0502365] [INSPIRE].

[61] R.G. Pereira et al., Dynamical spin structure factor for the anisotropic spin-1/2 Heisenberg chain, Phys. Rev. Lett. 96 (2006) 257202 [cond-mat/0603681].

[62] R.G. Pereira et al., Dynamical structure factor at small $q$ for the $X X Z$ spin-1/2 chain, J. Stat. Mech. Theory Exp. 08 (2007) P08022 [arXiv:0706.4327].

[63] J.S. Caux, P. Calabrese and N.A. Slavnov, One-particle dynamical correlations in the one-dimensional Bose gas, J. Stat. Mech. Theory Exp. 01 (2007) P01008 [cond-mat/0611321].

[64] A.G. Izergin, Partition function of the six-vertex model in a finite volume, Sov. Phys. Dokl. 32 (1987) 878.

[65] S. Pakuliak, V. Rubtsov and A. Silantyev, SOS model partition function and the elliptic weight functions, J. Phys. A 41 (2008) 295204 [arXiv:0802.0195].

[66] H. Rosengren, An Izergin-Korepin-type identity for the 8VSOS model, with applications to alternating sign matrices, Adv. Appl. Math. 43 (2009) 137.

[67] S. Kharchev and A. Zabrodin, Theta vocabulary I, J. Geom. Phys. 94 (2015) 19 [arXiv: 1502.04603].

[68] G. Niccoli and V. Terras, The 8-vertex model with quasi-periodic boundary conditions, J. Phys. A 49 (2016) 044001 [arXiv: 1508.0323]. 